\title{
A divergence-free low-order stabilized finite element method for a generalized steady state Boussinesq problem
}

\author{
Alejandro Allendes \\ Departamento de Matemática, Universidad Técnica Federico Santa María, Valparaíso, Chile. alejandro.allendes@usm.cl \\ Gabriel R. Barrenechea \\ Department of Mathematics and Statistics, University of Strathclyde, 26 Richmond Street, Glasgow G1 1XH, Scotland. \\ gabriel.barrenechea@strath.ac.uk \\ César Narranjo \\ Departamento de Matemática, Universidad Técnica Federico Santa María, Valparaíso, Chile. cesar.naranjo@alumnos.usm. cl
}

\begin{abstract}
In this work we propose and analyze a new stabilized finite element method for the coupled Navier-Stokes/temperature (or Boussinesq) equations. The method is built using low order conforming elements for velocity and temperature, and piecewise constant elements for pressure. With the help of the lowest order Raviart-Thomas space, a lifting of the jumps of the discrete pressure is built in such a way that when this lifting is added to the conforming velocity field, the resulting velocity is solenoidal (at the price of being non-conforming). This field is then fed to the momentum and temperature equations, guaranteeing that the convective terms in these equations are antisymmetric, without the need of altering them, thus simplifying the analysis of the resulting method. Existence of solutions, discrete stability, and optimal convergence are proved for both the conforming velocity field, and its corresponding divergence-free non-conforming counterpart. Numerical results confirm the theoretical findings, as well as the gain provided by the solenoidal discrete velocity field over the conforming one.
\end{abstract}

Keywords: Boussinesq problem; stabilized finite element method; divergence-free discrete velocity.

\section{Introduction}

In this work we aim at approximating incompressible, non-isothermal flows. We choose as a model problem a generalization of the well-known Boussinesq approximation (see [1, 2]). This problem, of high relevance in applications, has been the topic of a number of studies over the last few decades, and a vast number of works have been written about the solvability and approximation of it, using different approaches. We just mention in this, non-extensive, list of references the works $[3,4,5,6,7,8,9,10,11,12,13]$, and the references therein, as examples of the variety of approaches used for tackling this problem.

The particular model problem we choose is a steady-state generalized Boussinesq equation, where the viscosity and thermal conductivity are supposed to be temperature-dependent. The boundary conditions are homogeneous for the velocity, but the boundary datum for the temperature is not. This generalization has been the topic of multiple independent studies. In particular, in [14] the existence of solutions is shown using fixed point arguments (the uniqueness is also shown under an assumption of smallness of data and solutions), and these results rely strongly on two fundamental facts. First, a sufficiently small lifting of the boundary datum for the temperature can be proven to exist, in such a way that certain a priori estimates can be derived. Second, the velocity field is divergence-free, which allows the proof of the well-posedness of the fixed-point mapping, and a priori estimates for the fixed-point iterates.

The first restriction mentioned in the last paragraph, namely, the need for a sufficiently small lifting of the boundary datum for the temperature, can be proven if one of the two following assumptions follow (see [15, Section 4] for an in-deep discussion of this fact). First, it follows in a fairly straightforward way if this boundary datum is supposed 
to be small enough. Alternatively, since the norm in which the lifting needs to be small enough is only the $L^{3}$ norm (and thus does not include any derivatives), then this lifting can be proved to have a small enough norm if the mesh is sufficiently refined near the boundary. In such a case it is enough to build the lifting in the usual way, namely, nodal interpolation in the boundary nodes, and zero in all the interior nodes of the triangulation. This latter approach is the one followed in this work, and in our numerical experimentation this has been proven to be sufficient.

The second point raised ealier, namely, the need for the velocity to be divergence-free, is more delicate. As a matter of fact, one common point to most (if not all) discretizations of the Boussinesq problem is the need to guarantee an appropriate level of local mass conservation, as it has been shown that a poor local mass conservation affects the stability, and ultimately, the overall quality of the numerical results. This is especially true if low order conforming elements are used to approximate the velocity field, as the results in $[16,13]$ show. Then, several alternatives have been proposed. One possibility is to rewrite the convective term in its skew-symmetric form. In this way, even if the discrete velocity is not solenoidal (as it happens if conforming, low-order Lagrangian elements are used for velocity, as we use in this work), the convective term remains antisymmetric, thus not affecting stability (see, e.g., the recent works [17] and [10] for the use of this idea). Another possibility is to use divergence-free elements, such as the ones reviewed in the recent paper [18], where some preliminary results are also shown for a natural convection problem. Nevertheless, the applicability of these pairs to more challenging situations, and their stabilization to treat convectiondominated problems, is still to be carried out. A further alternative to produce exactly divergence-free finite element methods is to use BDM pairs of finite elements. This is the approach that has been followed in the fairly recent paper [15], where the analysis presented in [14] has been extended to cover the discrete setting. Nevertheless, some dG-like terms needed to be added to the formulation to enforce its stability, and also the number of degrees of freedom of BDM pairs is higher than for conforming methods.

In this work we propose a method using low order conforming finite element spaces. More precisely, we seek piecewise linear conforming velocities and temperatures, and piecewise constant pressures. Since this choice does not satisfy the inf-sup condition, the pressure is stabilized by penalizing inter-element jumps. It is well-known that the conforming piecewise linear velocity can not be solenoidal. Thus, we follow the idea presented in [19] where the internal jumps of the discrete pressure are used as degrees of freedom to build a unique element of the lowest order Raviart-Thomas space. This Raviart-Thomas field is then added to the conforming discrete velocity, and the enriched velocity thus built can be proved to be divergence-free. The price to pay for this is the loss of conformity, since this modified velocity field is only div-conforming, but this does not affect the analysis, since the only place in which this modification is used is in the convective terms, where only div-conformity is needed. In [19] this process was presented as a post-processing aimed at recovering a solenoidal discrete velocity at virtually zero cost, producing a discrete velocity that shared some advantages of a non-conforming element at the cost of a conforming one (i.e., needing fewer degrees of freedom). In this work this modification is at the core of the discrete method, since this modified (solenoidal) velocity field is the one fed to the momentum and temperature equations, thus avoiding any modifications of the convective terms. One final important property of this modified velocity field is that it can be proved to enjoy the same convergence properties as the conforming one, in a broken $H^{1}$-type norm. For this, we have modified and extended the arguments given in [19], where this fact had been proven for a particular case.

The rest of the manuscript is organized as follows. In Section 2 we present the main notations and prelimiary results used throughout. The model problem is presented in Section 3, where we review the main properties of the forms involved in the formulation, and the main results about existence of solutions. The finite element method proposed in this work is presented in Section 4, where we also show the existence of solutions under the appropriate smallness of the data or mesh fine enough near the boundary mentioned previously. The convergence of the method, and a priori error estimates, are proven in Section 5. For the existence and error analyses, we follow closely the general approach given in [18], modifying it when necessary to accommodate for the fact that we deal here with non-inf-sup

stable pairs of elements. The specific stabilizing terms used in the numerical experiments, and several numerical experiments confirming the theory and showing the good performance of the method are presented in Section 6, and some conclusion are drawn in Section 7.

\section{Preliminaries}

For an open domain $O \subset \mathbb{R}^{d}, d=2,3$, with Lipschitz boundary $\partial O, C(O)$ denotes the space of all continuous functions over $O$. For $r \geq 0$ and $p \in[1, \infty], L^{p}(O)$ and $W^{r, p}(O)$ denote the usual Lebesgue and Sobolev spaces 
endowed with the norms $\|\cdot\|_{L^{p}(O)}$ and $\|\cdot\|_{W^{r, p}(O)}$, respectively; if $p=2$, then $W^{r, p}(O)=H^{r}(O)$; the space $H_{0}^{1}(O)$ is the space of all functions in $H^{1}(O)$ with vanishing trace on $\partial O$; the space $L_{0}^{2}(O)$ is the space of all functions in $L^{2}(O)$ with zero mean value over $O$. The vector counterpart of all the previous spaces will be denoted in bold face and their matrix counterpart with an extra under accent, for example, $\boldsymbol{H}^{1}(O)=\left[H^{1}(O)\right]^{d}$ and $\boldsymbol{L}^{2}(O)=\left[L^{2}(O)\right]^{d \times d}$. In addition,

$\boldsymbol{H}(\mathbf{d i v}, O)$ stands for the space of $\boldsymbol{L}^{2}(O)$ functions whose divergence belongs to $L^{2}(\tilde{O})$ and $\boldsymbol{H}_{0}(\mathbf{d i v}, O)$ stands for the functions in $\boldsymbol{H}(\mathbf{d i v}, O)$ whose normal component vanishes at the boundary.

Now, we introduce all the notation related with the finite element nomenclature. Let $\mathscr{T}=\{K\}$ be a shape-regular partition of $\bar{\Omega}$ into simplices $K$, being either a triangle or a tetrahedron, as in [20], where $\Omega$ is a polytopal domain in $\mathbb{R}^{d}(d=2,3)$, with Lipschitz boundary $\Gamma$.

For a fixed partition $\mathscr{T}$ :

- $\mathcal{F}$ denotes the set of all element edges(2D)/faces(3D), $\mathcal{F}_{I} \subset \mathcal{F}$ denotes the set of interior edges(2D)/faces(3D), $\mathcal{F}_{\Gamma} \subset \mathcal{F}$ denotes the set of boundary edges(2D)/faces(3D);

- $\lambda_{n}$ denotes the piecewise linear basis function associated to the vertex $\boldsymbol{x}_{n}$, characterized by the condition $\lambda_{n}\left(\boldsymbol{x}_{m}\right)=\delta_{n m}$, where $\delta_{n m}$ denotes the Kronecker symbol.

For an element $K \in \mathscr{T}$ :

- $|K|$ denotes the area(2D)/volume(3D) of $K$ and $h_{K}$ denotes the diameter of $K$;

- $\mathbb{P}_{n}(K)$ denotes the space of polynomials on $K$ of total degree at most $n$;

- $\mathcal{F}_{K} \subset \mathcal{F}$ denotes the set containing the individual edges(2D)/faces(3D) of $K$;

- $\boldsymbol{n}_{\gamma}^{K}$ denotes the unit exterior normal vector to the edge(2D)/face(3D) $\gamma \in \mathcal{F}_{K}$;

- $\mathrm{v}_{\mid K}$ denotes the restriction of $\mathrm{v}$ to the element $K$.

Based on the previous definition, we let $h=\max _{K \in \mathscr{T}}\left\{h_{K}\right\}$.

For an edge(2D)/face(3D) $\gamma \in \mathcal{F}$ :

- $|\gamma|$ denotes the length(2D)/area(3D) of the edge(2D)/face(3D) $\gamma$ and $h_{\gamma}$ denotes the diameter of the edge(2D)/ face(3D) $\gamma$;

- $\Omega_{\gamma}:=\left\{K \in \mathscr{T}: \gamma \in \mathcal{F}_{K}\right\}$

- $\mathrm{v}_{\mid \gamma}$ denotes the restriction of $\mathrm{v}$ to the edge(2D)/face(3D) $\gamma$;

- $\varphi_{\gamma}$ denote the lowest order Raviart-Thomas basis function satisfying, for all $\gamma \in \mathcal{F}_{I} \cap \mathcal{F}_{K}$, that

$$
\boldsymbol{\varphi}_{\gamma}(\boldsymbol{x})= \pm \frac{|\gamma|}{d|K|}\left(\boldsymbol{x}-\boldsymbol{x}_{\gamma}\right), \quad \operatorname{div} \boldsymbol{\varphi}_{\gamma}= \pm \frac{|\gamma|}{|K|}, \quad \nabla \boldsymbol{\varphi}_{\gamma \mid K}= \pm \frac{|\gamma|}{d|K|} \boldsymbol{I} \quad \text { and } \quad\left\|\nabla \boldsymbol{\varphi}_{\gamma}\right\|_{\boldsymbol{\sim}^{2}(K)}^{2} \leq C h_{\gamma}^{d-2}
$$

where $\boldsymbol{x}_{\gamma}$ denotes the vertex opposite to the edge(2D)/face(3D) $\gamma, \boldsymbol{I}$ is the identity matrix, the signs \pm are chosen by fixing a normal vector for every $\gamma \in \mathcal{F}_{I}$, as depicted in Figure 1, and $C>0$ is independent of any mesh size; we also notice that $\boldsymbol{\varphi}_{\gamma \mid \gamma^{\prime}} \cdot \boldsymbol{n}_{\gamma^{\prime}}=0$, for all $\gamma, \gamma^{\prime} \in \mathcal{F}_{K}$, with $\gamma \neq \gamma^{\prime}$.

- $\llbracket \phi \rrbracket_{\gamma}$, on $\gamma=\mathcal{F}_{K^{+}} \cap \mathcal{F}_{K^{-}}$, denotes the jump of the function $\phi$, which is defined by fixing a normal vector for every $\gamma \in \mathcal{F}_{I}$, based on Figure 1 , as

$$
\llbracket \phi \rrbracket_{\gamma}=\phi_{K^{+}}-\phi_{K^{-}} .
$$

Based on all the previous definitions, we introduce the following broken Sobolev space

$$
H^{1}(\mathscr{T}):=\left\{\phi: \phi_{\mid K} \in H^{1}(K), \forall K \in \mathscr{T}\right\},
$$



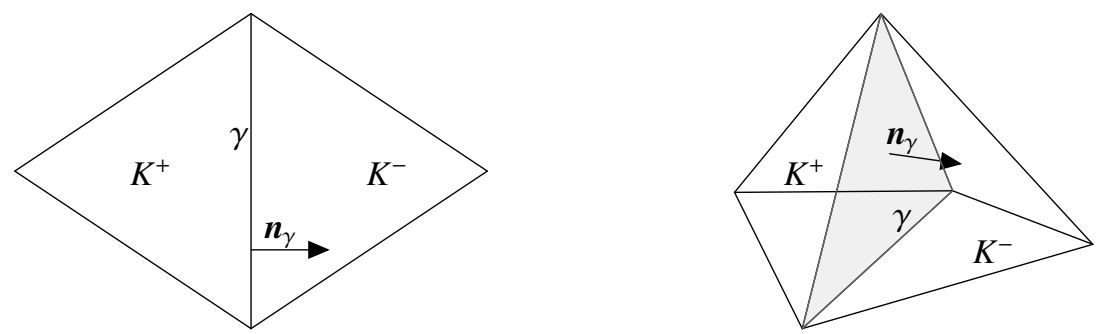

Figure 1: For $\gamma=\mathcal{F}_{K^{+}} \cap \mathcal{F}_{K^{-}}$, by fixing a normal vector $\boldsymbol{n}_{\gamma}$ pointing from $K^{+}$to $K^{-}$, the lowest order Raviart-Thomas basis function and inter element jumps are defined.

and also the following finite element spaces,

$$
\begin{gathered}
V(\mathscr{T}):=\left\{v \in C(\bar{\Omega}): v_{\mid K} \in \mathbb{P}_{1}(K), \forall K \in \mathscr{T}\right\}, \quad V_{0}(\mathscr{T}):=V(\mathscr{T}) \cap H_{0}^{1}(\Omega), \\
Q(\mathscr{T}):=\left\{q \in L_{0}^{2}(\Omega): q_{\mid K} \in \mathbb{P}_{0}(K), \forall K \in \mathscr{T}\right\} .
\end{gathered}
$$

The Lagrange interpolator operator $i_{h}$ onto $V(\mathscr{T})$ satisfies [21, Theorem 4.4.20]

$$
\left\|\xi-i_{h}(\xi)\right\|_{L^{2}(\Omega)}+h\left\|\nabla\left(\xi-i_{h}(\xi)\right)\right\|_{L^{2}(\Omega)} \leq C h^{2}\|\xi\|_{H^{2}(\Omega)} \quad \forall \xi \in H^{2}(\Omega),
$$

with its obvious extension to vector-valued functions. Similarly, we define the orthogonal $L^{2}$-projection operator $\Pi_{\mathscr{T}}$, onto the space of piecewise constant functions defined by

$$
\Pi_{\mathscr{T}}(\phi)_{\mid K}=\frac{1}{|K|} \int_{K} \phi \quad \forall K \in \mathscr{T} .
$$

We will also make use of the following Poincaré $[22,23,24]$ and embedding inequalities [25, Theorem 1.3 in Chapter I] valid for any $\xi \in H_{0}^{1}(\Omega)$, any $\vartheta \in H^{1}(\Omega)$, and $p \in[1, \infty)$ if $d=2$ and $p \in[1,6]$ if $d=3$ :

$$
\|\xi\|_{L^{2}(\Omega)} \leq C_{p}\|\nabla \xi\|_{L^{2}(\Omega)}, \quad\left\|\vartheta-\Pi_{\mathscr{T}}(\vartheta)\right\|_{L^{2}(\Omega)} \leq C h\|\nabla \vartheta\|_{L^{2}(\Omega)}, \quad\|\vartheta\|_{L^{p}(\Omega)} \leq C_{e m b}\|\vartheta\|_{H^{1}(\Omega)}
$$

with their obvious extensions to vector-valued functions. Also, we will often use the constant $\tilde{C}_{p}=\left(1+C_{p}^{2}\right)^{1 / 2}$. We will make use of the local trace inequality [21, Equation 10.3.8] (with its obvious extension to vector-valued functions): There exists $C>0$, independent of $h$, such that, for all $K \in \mathscr{T}$ and all $\gamma \in \mathcal{F}_{K}$ :

$$
h_{\gamma}^{-1}\|\chi\|_{L^{2}(\gamma)}^{2} \leq C\left(h_{K}^{-2}\|\chi\|_{L^{2}(K)}^{2}+\|\nabla \chi\|_{L^{2}(K)}^{2}\right) \quad \forall \chi \in H^{1}(K) .
$$

As a consequence of the last inequality, and using (4), there exists a constant $C>0$ such that, for all $\chi \in H^{1}(\Omega)$

$$
\left[\sum_{\gamma \in \mathcal{F}_{I}} h_{\gamma}\left\|\llbracket \chi-\Pi_{\mathscr{T}}(\chi)\right\|_{\gamma} \|_{L^{2}(\gamma)}^{2}\right]^{1 / 2} \leq C h\|\nabla \chi\|_{L^{2}(\Omega)} .
$$

Finally, in the manuscript we shall use $C$ to denote any positive constant which is independent of any mesh size, and whose value may change whenever it is written in two different places.

\section{Model problem}

We are interested in the study of the following generalized Boussinesq problem: given prescribed data $\mathbf{g}$ and $\mathbf{t}_{D}$, find $(\mathbf{u}, p, t)$ such that

$$
\left\{\begin{array}{rccc}
-\operatorname{div}(\varepsilon(\mathrm{t}) \nabla \mathbf{u})+\mathbf{u} \cdot(\nabla \mathbf{u})+\nabla \mathrm{p}-\mathbf{g t} & =\mathbf{0} & \text { in } \Omega, \\
\operatorname{div} \mathbf{u} & = & & \text { in } \Omega, \\
-\operatorname{div}(\kappa(\mathrm{t}) \nabla \mathrm{t})+\mathbf{u} \cdot \nabla \mathrm{t} & = & & \text { in } \Omega, \\
\mathbf{u} & =\mathbf{0} & \text { on } \Gamma, \\
\mathrm{t} & =\mathrm{t}_{D} & & \text { on } \Gamma .
\end{array}\right.
$$


In (7), $\mathbf{u}$ denotes the fluid velocity, $\mathrm{p}$ the pressure, and the temperature. The data of the problem is such that $\mathbf{g} \in \boldsymbol{L}^{2}(\Omega), \mathrm{t}_{D} \in H^{1 / 2}(\Gamma) \cap C(\Gamma)$, and the functions $\varepsilon(\cdot)$ and $\kappa(\cdot)$ are the fluid viscosity and the thermal conductivity, respectively, that are supposed to satisfy

$$
\begin{gathered}
\left|\varepsilon\left(\mathrm{t}_{1}\right)-\varepsilon\left(\mathrm{t}_{2}\right)\right| \leq \varepsilon_{l i p}\left|\mathrm{t}_{1}-\mathrm{t}_{2}\right|, \quad\left|\kappa\left(\mathrm{t}_{1}\right)-\kappa\left(\mathrm{t}_{2}\right)\right| \leq \kappa_{l i p}\left|\mathrm{t}_{1}-\mathrm{t}_{2}\right|, \quad \text { a.e. in } \Omega, \forall \mathrm{t}_{1}, \mathrm{t}_{2} \in H^{1}(\Omega), \\
0<\varepsilon_{1} \leq \varepsilon(\mathrm{t}) \leq \varepsilon_{2}, \quad 0<\kappa_{1} \leq \kappa(\mathrm{t}) \leq \kappa_{2}, \quad \text { a.e. in } \Omega, \forall \mathrm{t} \in H^{1}(\Omega) .
\end{gathered}
$$

The weak form of (7) reads as follows: find $(\mathbf{u}, \mathrm{p}, \mathrm{t}) \in \boldsymbol{H}_{0}^{1}(\Omega) \times L_{0}^{2}(\Omega) \times H^{1}(\Omega)$ such that $\mathrm{t}_{\mid \Gamma}=\mathrm{t}_{D}$ and

$$
\left\{\begin{array}{rlll}
\mathcal{A}_{F}(\mathrm{t} ; \mathbf{u}, \boldsymbol{\xi})+C_{F}(\mathbf{u} ; \mathbf{u}, \boldsymbol{\xi})-\mathcal{B}_{F}(\boldsymbol{\xi}, \mathrm{p})-\mathcal{G}_{F}(\mathrm{t}, \boldsymbol{\xi}) & =0 & \forall \boldsymbol{\xi} \in \boldsymbol{H}_{0}^{1}(\Omega), \\
\mathcal{B}_{F}(\mathbf{u}, \phi) & =0 & \forall \phi \in L_{0}^{2}(\Omega), \\
\mathcal{A}_{T}(\mathrm{t} ; \mathrm{t}, \psi)+C_{T}(\mathbf{u} ; \mathrm{t}, \psi) & = & 0 & \forall \psi \in H_{0}^{1}(\Omega),
\end{array}\right.
$$

where the respective forms are defined as

$$
\begin{aligned}
& \mathcal{A}_{F}(\phi ; \boldsymbol{\xi}, \boldsymbol{\zeta}):=\int_{\Omega} \varepsilon(\phi) \nabla \boldsymbol{\xi}: \nabla \boldsymbol{\zeta}, \quad C_{F}(\boldsymbol{\vartheta} ; \boldsymbol{\xi}, \boldsymbol{\zeta}):=\int_{\Omega}(\boldsymbol{\vartheta} \cdot \nabla \boldsymbol{\xi}) \cdot \boldsymbol{\zeta}, \quad \mathcal{B}_{F}(\boldsymbol{\xi}, \phi):=\int_{\Omega} \phi \operatorname{div} \boldsymbol{\xi} \\
& \mathcal{G}_{F}(\phi, \boldsymbol{\xi}):=\int_{\Omega} \phi \mathbf{g} \cdot \boldsymbol{\xi}, \quad \mathcal{A}_{T}(\phi ; \varphi, \psi):=\int_{\Omega} \kappa(\phi) \nabla \varphi \cdot \nabla \psi, \quad C_{T}(\boldsymbol{\xi} ; \varphi, \psi):=\int_{\Omega}(\boldsymbol{\xi} \cdot \nabla \varphi) \psi
\end{aligned}
$$

In [14] a detailed analysis of (9) is presented, and the existence of a solution is proven under all the above assumptions. The main tool of the proof is a splitting of the temperature as

$$
\mathrm{t}=\mathrm{t}_{0}+\mathrm{t}_{1}, \quad \text { with } \quad \mathrm{t}_{0} \in H_{0}^{1}(\Omega), \quad \text { and } \quad \mathrm{t}_{1} \in H^{1}(\Omega) \text { such that } \mathrm{t}_{1 \mid \Gamma}=\mathrm{t}_{D} .
$$

Then, using that the lifting $t_{1}$ can be chosen arbitrary small, it is proven that the problem (9) has a solution $(\mathbf{u}, p, t) \in$ $\boldsymbol{H}_{0}^{1}(\Omega) \times L_{0}^{2}(\Omega) \times H^{1}(\Omega)$ with $\mathrm{t}=\mathrm{t}_{0}+\mathrm{t}_{1}$, and the following continuous dependency holds

$$
\|\mathbf{u}\|_{H^{1}(\Omega)} \leq \mathrm{C}_{\mathbf{u}}\left\|\mathrm{t}_{1}\right\|_{H^{1}(\Omega)} \quad \text { and } \quad\|\mathrm{t}\|_{H^{1}(\Omega)} \leq \mathrm{C}_{\mathrm{t}}\left\|\mathrm{t}_{1}\right\|_{H^{1}(\Omega)}
$$

where $C_{\mathbf{u}}$ y $C_{\mathrm{t}}$ are positive constants independent of the lifting $\mathrm{t}_{1}$. Furthermore, in [15, Theorem 2.3] a uniqueness result is proved under a smallness assumption on the data, relaxing some geometrical hypotheses over the domain required in [14].

Remark 1. The model problem presented in this work is not, strictly speaking, the Oberbeck-Boussinesq approximation. In fact, for such an approximation to be valid, the values of $\varepsilon$ and $\kappa$ would neeed to be constant in space, and the temperature differences need to be small. In the last decade work has been carried out exploring the limits of the Boussinesq approximation. An exhaustive study of this issue is beyond the scope of this work, but we refer to the works [26, 27] where extensive numerical studies are presented and a thorough discussion of this issue is presented.

\subsection{Stability properties}

This section is devoted to list a series of inf-sup, continuity and coercivity properties of all the forms given in (10).

Lemma 1. There exist $C_{c o, F}, C_{c o, T}>0$ such that, for all $\boldsymbol{\xi} \in \boldsymbol{H}_{0}^{1}(\Omega)$, and all $\varphi, \psi \in H^{1}(\Omega)$, the following ellipticities hold

$$
\mathcal{A}_{F}(\varphi ; \xi, \xi) \geq C_{c o, F}\|\boldsymbol{\xi}\|_{\boldsymbol{H}^{1}(\Omega)}^{2}, \quad \mathcal{A}_{T}(\varphi ; \psi, \psi) \geq C_{c o, T}\|\psi\|_{H^{1}(\Omega)}^{2} .
$$

Moreover, there exists $\beta>0$ such that the following inf-sup condition holds

$$
\sup _{\boldsymbol{\zeta} \in \boldsymbol{H}_{0}^{1}(\Omega) \backslash\{\boldsymbol{0}\}} \frac{\mathcal{B}_{F}(\boldsymbol{\zeta}, \phi)}{\|\zeta\|_{\boldsymbol{H}^{1}(\Omega)}} \geq \beta\|\phi\|_{L^{2}(\Omega)} \quad \forall \phi \in L_{0}^{2}(\Omega) .
$$

Proof. The ellipticity properties can be obtained by using (8) and the Poincaré inequality. The inf-sup condition is proven in [25, Section 5.1 in Chapter I]. 
Lemma 2. Let $\boldsymbol{\xi}, \zeta, \boldsymbol{\vartheta} \in \boldsymbol{H}^{1}(\Omega), \chi \in L^{2}(\Omega)$ and $\theta, \psi, \varphi \in H^{1}(\Omega)$. Then, it follows that

$$
\begin{array}{rlrl}
\left|\mathcal{A}_{F}(\theta ; \boldsymbol{\xi}, \boldsymbol{\zeta})\right| & \leq \varepsilon_{2}\|\boldsymbol{\xi}\|_{\boldsymbol{H}^{1}(\Omega)}\|\boldsymbol{\zeta}\|_{\boldsymbol{H}^{1}(\Omega)}, & \left|\mathcal{A}_{T}(\varphi ; \theta, \psi)\right| \leq \kappa_{2}\|\theta\|_{H^{1}(\Omega)}\|\psi\|_{H^{1}(\Omega)}, \\
\left|C_{F}(\boldsymbol{\vartheta} ; \boldsymbol{\xi}, \boldsymbol{\zeta})\right| \leq C_{C}\|\boldsymbol{\vartheta}\|_{\boldsymbol{H}^{1}(\Omega)}\|\boldsymbol{\xi}\|_{\boldsymbol{H}^{1}(\Omega)}\|\boldsymbol{\zeta}\|_{\boldsymbol{H}^{1}(\Omega)}, & \left|C_{T}(\boldsymbol{\vartheta} ; \theta, \psi)\right| \leq C_{\mathrm{c}}\|\boldsymbol{\vartheta}\|_{\boldsymbol{H}^{1}(\Omega)}\|\theta\|_{H^{1}(\Omega)}\|\psi\|_{H^{1}(\Omega)} \\
\left|\mathcal{B}_{F}(\boldsymbol{\xi}, \chi)\right| \leq d\|\boldsymbol{\xi}\|_{\boldsymbol{H}^{1}(\Omega)}\|\chi\|_{L^{2}(\Omega)}, & \left|\mathcal{G}_{F}(\theta, \boldsymbol{\xi})\right| \leq C_{g}\|\boldsymbol{g}\|_{\boldsymbol{L}^{2}(\Omega)}\|\theta\|_{H^{1}(\Omega)}\|\boldsymbol{\xi}\|_{\boldsymbol{H}^{1}(\Omega)},
\end{array}
$$

where $C_{C}, C_{\mathfrak{c}}, C_{g}$ are positive constants. Also, if $\theta_{1}, \theta_{2} \in H^{1}(\Omega), \zeta \in W^{1, \infty}(\Omega)$ and $\phi \in W^{1, \infty}(\Omega)$, then

$$
\begin{aligned}
\left|\mathcal{A}_{F}\left(\theta_{1} ; \boldsymbol{\zeta}, \boldsymbol{\xi}\right)-\mathcal{A}_{F}\left(\theta_{2} ; \boldsymbol{\zeta}, \boldsymbol{\xi}\right)\right| & \leq C_{\varepsilon, l i p}\|\zeta\|_{W^{1, \infty}(\Omega)}\left\|\theta_{1}-\theta_{2}\right\|_{L^{2}(\Omega)}\|\boldsymbol{\xi}\|_{\boldsymbol{H}^{1}(\Omega)}, \\
\left|\mathcal{A}_{T}\left(\theta_{1} ; \phi, \psi\right)-\mathcal{A}_{T}\left(\theta_{2} ; \phi, \psi\right)\right| & \leq C_{\kappa, l i p}\|\phi\|_{W^{1, \infty}(\Omega)}\left\|\theta_{1}-\theta_{2}\right\|_{L^{2}(\Omega)}\|\psi\|_{H^{1}(\Omega)}
\end{aligned}
$$

Proof. All the results are obtained using Hölder inequalities, the Lipchitz continuity of $\varepsilon$ and $\kappa$, and the fact that $H^{1}(\Omega)$ is continuously embedded in $L^{p}(\Omega)$ for $p \leq 6$.

Finally, defining the space $\boldsymbol{X}:=\{\boldsymbol{\xi} \in \boldsymbol{H}(\operatorname{div}, O): \operatorname{div} \boldsymbol{\xi}=0\}$, then integration by parts allows us to conclude that, for all $\xi \in X$,

$$
\begin{aligned}
& C_{F}(\boldsymbol{\xi} ; \boldsymbol{\zeta}, \boldsymbol{\vartheta})=-C_{F}(\boldsymbol{\xi} ; \boldsymbol{\vartheta}, \boldsymbol{\zeta}) \quad \forall \boldsymbol{\zeta} \in \boldsymbol{H}^{1}(\Omega), \forall \boldsymbol{\vartheta} \in \boldsymbol{H}_{0}^{1}(\Omega), \\
& \mathcal{C}_{F}(\boldsymbol{\xi} ; \boldsymbol{\zeta}, \boldsymbol{\zeta})=0 \quad \forall \boldsymbol{\zeta} \in \boldsymbol{H}_{0}^{1}(\Omega), \\
& C_{T}(\boldsymbol{\xi} ; \theta, \psi)=-C_{T}(\boldsymbol{\xi} ; \psi, \theta) \quad \forall \theta \in H^{1}(\Omega), \forall \psi \in H_{0}^{1}(\Omega), \\
& C_{T}(\boldsymbol{\xi} ; \psi, \psi)=0 \quad \forall \psi \in H_{0}^{1}(\Omega) .
\end{aligned}
$$

\section{Finite element discretization}

We start presenting the finite element method analyzed in this work: find $\left(\mathbf{u}_{\mathscr{T}}, \mathrm{p}_{\mathscr{T}}, \mathrm{t}_{\mathscr{T}}\right) \in V_{0}(\mathscr{T}) \times Q(\mathscr{T}) \times V(\mathscr{T})$ such that $\mathrm{t}_{\mathscr{T} \mid \Gamma}=i_{h}\left(\mathrm{t}_{D}\right)$ and that

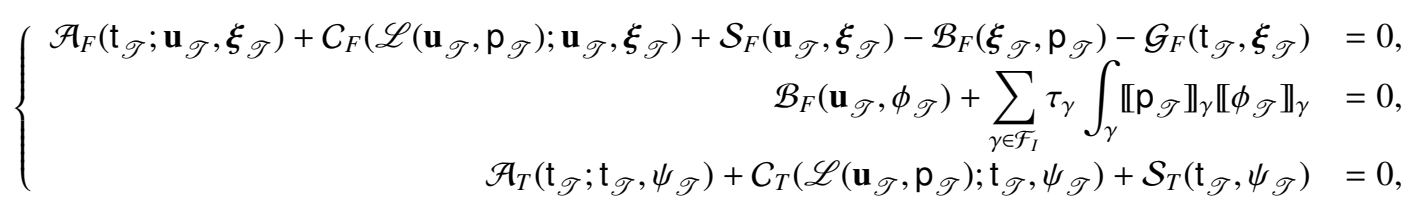

for all $\left(\xi_{\mathscr{T}}, \phi_{\mathscr{T}}, \psi_{\mathscr{T}}\right) \in \boldsymbol{V}_{0}(\mathscr{T}) \times Q(\mathscr{T}) \times V_{0}(\mathscr{T})$. For the stabilization parameter, we have followed the expression given in [19] (obtained as the result of an enrichment strategy) and we set

$$
\tau_{\gamma}=\frac{h_{\gamma}}{12} \quad \forall \gamma \in \mathcal{F}_{I}
$$

The operator $\mathscr{L}$ is defined as

$$
\mathscr{L}\left(\mathbf{u}_{\mathscr{T}}, \mathrm{p}_{\mathscr{T}}\right):=\mathbf{u}_{\mathscr{T}}+\sum_{\gamma \in \mathcal{F}_{I}} \tau_{\gamma} \llbracket \mathrm{p}_{\mathscr{T}} \rrbracket_{\gamma} \boldsymbol{\varphi}_{\gamma},
$$

where $\varphi_{\gamma}$ is defined in (1). For the moment, $\mathcal{S}_{F}$ and $\mathcal{S}_{T}$ are generic stabilizing bilinear forms that are supposed to satisfy

- $\mathcal{S}_{F}$ and $\mathcal{S}_{T}$ are symmetric;

- $\mathcal{S}_{F}(\boldsymbol{\xi}, \boldsymbol{\xi}) \geq 0$ and $\mathcal{S}_{T}(\psi, \psi) \geq 0$, for all $\boldsymbol{\xi}$ and $\psi$ regular enough such that these bilinear forms can be evaluated;

- $\mathcal{S}_{F}$ and $\mathcal{S}_{T}$ are such that, for all $\boldsymbol{\xi} \in \boldsymbol{H}^{2}(\Omega)$ and all $\psi \in H^{2}(\Omega)$,

$$
\mathcal{S}_{F}\left(\boldsymbol{\xi}-i_{h}(\boldsymbol{\xi}), \boldsymbol{\xi}-i_{h}(\boldsymbol{\xi})\right) \leq C h^{2}\|\boldsymbol{\xi}\|_{\boldsymbol{H}^{2}(\Omega)}^{2} \quad \text { and } \quad \mathcal{S}_{T}\left(\psi-i_{h}(\psi), \psi-i_{h}(\psi)\right) \leq C h^{2}\|\psi\|_{H^{2}(\Omega)}^{2}
$$

Moreover, we assume that, if the solution of (7) satisfies $\mathbf{u} \in \boldsymbol{H}^{2}(\Omega)$ and all $t \in H^{2}(\Omega)$, then the stabilizing forms satisfy

$$
\mathcal{S}_{F}(\mathbf{u}, \mathbf{u}) \leq C h^{2}\|\mathbf{u}\|_{H^{2}(\Omega)}^{2} \quad \text { and } \quad \mathcal{S}_{T}(\mathrm{t}, \mathrm{t}) \leq C h^{2}\|\mathrm{t}\|_{H^{2}(\Omega)}^{2} .
$$


From the previous hypotheses, the following Cauchy-Schwarz inequalities can be proven, for any $\boldsymbol{\xi}, \boldsymbol{\zeta}$ and $\theta, \psi$ regular enough so the bilinear forms can be evaluated:

$$
\left|\mathcal{S}_{F}(\boldsymbol{\xi}, \zeta)\right| \leq \mathcal{S}_{F}(\boldsymbol{\xi}, \boldsymbol{\xi})^{1 / 2} \mathcal{S}_{F}(\boldsymbol{\zeta}, \zeta)^{1 / 2} \quad \text { and } \quad\left|\mathcal{S}_{T}(\theta, \psi)\right| \leq \mathcal{S}_{T}(\theta, \theta)^{1 / 2} \mathcal{S}_{T}(\psi, \psi)^{1 / 2}
$$

Remark 2. To keep consistency in the physical units, the stabilization parameter given by (16) should depend on $\varepsilon^{-1}$. To simplify the presentation, and to avoid including a further nonlinearity in the method, we propose the following expression for

$$
\tau_{\gamma}:=\frac{h_{\gamma}}{12 \varepsilon_{0}},
$$

where $\varepsilon_{0}$ is a reference viscosity, chosen globally. In our numerical experiments below we have chosen $\varepsilon_{0}=1$, and thus we perform the analysis for this value. Nevertheless, we should keep in mind that (21) is the expression that should be considered for physical consistency.

As we mentioned in the introduction, one of the main properties of the method that we are proposing is that it delivers a divergence-free velocity field at virtually zero cost. This fact is stated in the next result.

Theorem 1. Let $\left(\mathbf{u}_{\mathscr{T}}, \mathrm{p}_{\mathscr{T}}, \mathrm{t}_{\mathscr{T}}\right)$ be a solution of (15) and let $\mathscr{L}$ be defined in (17). Then,

$$
\operatorname{div} \mathscr{L}\left(\mathbf{u}_{\mathscr{T}}, \mathrm{p}_{\mathscr{T}}\right)=0 \quad \text { in } \Omega
$$

Proof. Let $\phi_{\mathscr{T}} \in Q(\mathscr{T})$. From the definition of $\mathscr{L}$ in (17), and the second equation in (15) it follows that

$$
\int_{\Omega} \operatorname{div} \mathscr{L}\left(\mathbf{u}_{\mathscr{T}}, \mathrm{p}_{\mathscr{T}}\right) \phi_{\mathscr{T}}=-\sum_{\gamma \in \mathcal{F}_{I}} \tau_{\gamma} \int_{\gamma} \llbracket \mathrm{p}_{\mathscr{T}} \rrbracket_{\gamma} \llbracket \phi_{\mathscr{T}} \rrbracket_{\gamma}+\sum_{K \in \mathscr{T}} \int_{K} \sum_{\gamma \in \mathcal{F}_{I} \cap \mathcal{F}_{K}} \tau_{\gamma} \llbracket \mathrm{p}_{\mathscr{T}} \rrbracket_{\gamma} \operatorname{div} \boldsymbol{\varphi}_{\gamma} \phi_{\mathscr{T}}
$$

Now, using the fact that $\phi_{\mathscr{T} \mid K} \in \mathbb{P}_{0}(K)$, integration by parts, (1) and (2), allow us to conclude that

$$
\begin{aligned}
\sum_{K \in \mathscr{T}} \int_{K} \sum_{\gamma \in \mathcal{F}_{I} \cap \mathcal{F}_{K}} \tau_{\gamma} \llbracket p_{\mathscr{T}} \rrbracket_{\gamma} \operatorname{div} \boldsymbol{\varphi}_{\gamma} \phi_{\mathscr{T}} & =\sum_{K \in \mathscr{T}} \sum_{\gamma^{\prime} \in \mathcal{F}_{K}} \int_{\gamma^{\prime}} \sum_{\gamma \in \mathcal{F}_{I} \cap \mathcal{F}_{K}} \tau_{\gamma} \llbracket p_{\mathscr{T}} \rrbracket_{\gamma} \boldsymbol{\varphi}_{\gamma} \cdot \boldsymbol{n}_{\gamma^{\prime}}^{K} \phi_{\mathscr{T}} \\
& =\sum_{\gamma \in \mathcal{F}_{I}} \tau_{\gamma} \int_{\gamma} \llbracket p_{\mathscr{T}} \rrbracket_{\gamma} \llbracket \phi_{\mathscr{T}} \rrbracket_{\gamma} .
\end{aligned}
$$

Therefore, $\operatorname{div} \mathscr{L}\left(\mathbf{u}_{\mathscr{T}}, \mathrm{p}_{\mathscr{T}}\right) \in Q(\mathscr{T})^{\perp}$. Now, we will prove that $\operatorname{div} \mathscr{L}\left(\mathbf{u}_{\mathscr{T}}, \mathrm{p}_{\mathscr{T}}\right) \in Q(\mathscr{T})$. In fact, note that the enriched field $\left.\mathscr{L}\left(\mathbf{u}_{\mathscr{T}}, \mathrm{p}_{\mathscr{T}}\right)\right|_{K} \in \mathbb{P}_{1}(K)$ for all $K \in \mathscr{T}$ and that $\mathscr{L}\left(\mathbf{u}_{\mathscr{T}}, \mathrm{p}_{\mathscr{T}}\right) \in \boldsymbol{H}(\operatorname{div}, \Omega)$. Then, $\left.\operatorname{div} \mathscr{L}\left(\mathbf{u}_{\mathscr{T}}, \mathrm{p}_{\mathscr{T}}\right)\right|_{K} \in$ $\mathbb{P}_{0}(K)$. On the other hand, using the Divergence Theorem, the fact that $\mathbf{u}_{\mathscr{T}} \in \boldsymbol{V}_{0}(\mathscr{T})$, (1), and, for $\gamma \in \mathcal{F}_{I}, \boldsymbol{\varphi}_{\gamma} \cdot \boldsymbol{n}_{\Gamma}=0$ on $\Gamma$, we obtain

$$
\int_{\Omega} \operatorname{div} \mathscr{L}\left(\mathbf{u}_{\mathscr{T}}, \mathrm{p}_{\mathscr{T}}\right)=\int_{\Gamma} \mathscr{L}\left(\mathbf{u}_{\mathscr{T}}, \mathrm{p}_{\mathscr{T}}\right) \cdot \boldsymbol{n}_{\Gamma}=\int_{\Gamma} \mathbf{u}_{\mathscr{T}} \cdot \boldsymbol{n}_{\Gamma}+\int_{\Gamma} \sum_{K \in \mathscr{T}} \sum_{\gamma \in \mathscr{F}_{I} \cap \mathcal{F}_{K}} \tau_{\gamma} \llbracket \mathrm{p}_{\mathscr{T}} \mathbb{1}_{\gamma} \boldsymbol{\varphi}_{\gamma} \cdot \boldsymbol{n}_{\Gamma}=0
$$

where $\boldsymbol{n}_{\Gamma}$ is the unit exterior normal vector to $\Gamma$. Therefore, $\int_{\Omega} \operatorname{div} \mathscr{L}\left(\mathbf{u}_{\mathscr{T}}, \mathrm{p}_{\mathscr{T}}\right)=0$. Thus, $\operatorname{div} \mathscr{L}\left(\mathbf{u}_{\mathscr{T}}, \mathrm{p}_{\mathscr{T}}\right) \in$ $Q(\mathscr{T}) \cap Q(\mathscr{T})^{\perp}$, which implies that $\operatorname{div} \mathscr{L}\left(\mathbf{u}_{\mathscr{T}}, \mathrm{p}_{\mathscr{T}}\right)=0$ in $\Omega$.

Remark 3. The divergence-free velocity field $\mathscr{L}\left(\mathbf{u}_{\mathscr{T}}, \mathrm{p}_{\mathscr{T}}\right)$ given in (17) is non-conforming, in the sense that it does not belong to $\boldsymbol{H}^{1}(\Omega)$. Nevertheless, it belongs to $\boldsymbol{H}(\mathbf{d i v}, \Omega)$ since it is the sum of a continuous piecewise linear function and an element of the global Raviart-Thomas space, so the antisymmetry of the convective terms remain valid.

This construction for the velocity field has been proposed earlier in the context of stabilization of the lowest order pair for the Stokes problem, see [19]. In there, this field was presented as a post-process of the discrete solution that guaranteed local mass conservation at no cost. Thus, preserving the solenoidal character of the exact velocity using fewer degrees of freedom. In the present method, rather than appearing as a postprocess of the discrete solution, the field $\mathscr{L}\left(\mathbf{u}_{\mathscr{T}}, \mathrm{p}_{\mathscr{T}}\right)$ is hard-wired into the definition of the method itself, and is of fundamental importance in the stability analysis of (15). 


\subsection{Discrete stability and coercivity properties}

In this section we state the main stability properties of all the different forms appearing in (10), whose proofs will be postponed to the Appendix section 8 . The analysis will be done using the following mesh-dependent norms, defined for all $\boldsymbol{\xi}, \phi$ and $\psi$ smooth enough, as:

$$
\begin{aligned}
\|(\boldsymbol{\xi}, \phi)\|_{\mathcal{N}(\mathscr{T})}^{2} & =\varepsilon_{1}\|\nabla \boldsymbol{\xi}\|_{\boldsymbol{\sim}^{2}(\Omega)}^{2}+\mathcal{S}_{F}(\boldsymbol{\xi}, \boldsymbol{\xi})+\sum_{\gamma \in \mathcal{F}_{I}} \tau_{\gamma}\|\| \phi \rrbracket \|_{L^{2}(\gamma)}^{2}, \\
\|\psi\|_{\mathcal{T}(\mathscr{T})}^{2} & =\kappa_{1}\|\psi\|_{H^{1}(\Omega)}^{2}+\mathcal{S}_{T}(\psi, \psi),
\end{aligned}
$$

where the constants $\varepsilon_{1}$ and $\kappa_{1}$ are given in (8). To avoid unnecessary complications and diversions, from now on every time we evaluate one of these norms of a given function, we will suppose the function is regular enough for these norms to be evaluated. For example, the sentence "for every $\psi \in H^{1}(\Omega),\|\psi\|_{\mathcal{T}(\mathscr{T})}^{2}$ is bounded by..." supposes that $\psi \in H^{1}(\Omega)$ and, in addition, it is regular enough for $\mathcal{S}_{T}(\psi, \psi)$ to be finite.

Lemma 3. The following inequalities hold, for all $\boldsymbol{\xi} \in \boldsymbol{H}_{0}^{1}(\Omega), \phi_{\mathscr{T}} \in Q(\mathscr{T})$ and $q \in[1,6]$,

$$
\begin{aligned}
\left(\sum_{K \in \mathscr{T}}\left\|\nabla \mathscr{L}\left(\boldsymbol{\xi}, \phi_{\mathscr{T}}\right)\right\|_{\underset{\sim}{L^{2}(K)}}^{2}\right)^{1 / 2} \leq C\left(\|\nabla \boldsymbol{\xi}\|_{\underset{\sim}{L^{2}(\Omega)}}^{2}+\sum_{\gamma \in \mathcal{F}_{I}} \tau_{\gamma}\left\|\llbracket \phi_{\mathscr{T}} \rrbracket\right\|_{L^{2}(\gamma)}^{2}\right)^{1 / 2} . \\
\left\|\mathscr{L}\left(\boldsymbol{\xi}, \phi_{\mathscr{T}}\right)\right\|_{L^{q}(\Omega)} \leq \bar{C}_{q}\left\|\left(\boldsymbol{\xi}, \phi_{\mathscr{T}}\right)\right\|_{\mathcal{N}(\mathscr{T})},
\end{aligned}
$$

Proof. See the Appendix.

Lemma 4. The following inequalities hold, for all $\boldsymbol{\xi} \in \boldsymbol{H}_{0}^{1}(\Omega) \cap \boldsymbol{W}^{1, \infty}(\Omega),(\boldsymbol{\vartheta}, \chi) \in\left\{(\boldsymbol{\zeta}, \phi) \in \boldsymbol{H}_{0}^{1}(\Omega) \times\left[H^{1}(\mathscr{T}) \times\right.\right.$ $\left.\left.L_{0}^{2}(\Omega)\right]: \operatorname{div} \mathscr{L}(\boldsymbol{\zeta}, \phi)=0\right\}, \theta \in W^{1, \infty}(\Omega), \psi \in H^{1}(\Omega), \boldsymbol{\xi}_{\mathscr{T}}, \boldsymbol{\zeta}_{\mathscr{T}} \in \boldsymbol{V}_{0}(\Omega), \phi_{\mathscr{T}} \in Q(\mathscr{T}), \vartheta_{\mathscr{T}}, \theta_{\mathscr{T}}, \psi_{\mathscr{T}} \in V(\mathscr{T})$, $\left(\boldsymbol{\vartheta}_{\mathscr{T}}, \chi_{\mathscr{T}}\right) \in\left\{\left(\omega_{\mathscr{T}}, \pi_{\mathscr{T}}\right) \in \boldsymbol{V}_{0}(\mathscr{T}) \times Q(\mathscr{T}): \operatorname{div} \mathscr{L}\left(\omega_{\mathscr{T}}, \pi_{\mathscr{T}}\right)=0\right\}:$

$$
\begin{aligned}
\left|\mathcal{G}_{F}\left(\theta, \boldsymbol{\xi}_{\mathscr{T}}\right)\right| & \leq \tilde{C}_{g}\|\mathbf{g}\|_{L^{2}(\Omega)}\|\theta\|_{\mathcal{T}(\mathscr{T})}\left\|\left(\boldsymbol{\xi}_{\mathscr{T}}, \phi_{\mathscr{T}}\right)\right\|_{\mathcal{N}(\mathscr{T})}, \\
\left|\mathcal{A}_{T}\left(\psi ; \theta_{\mathscr{T}}, \psi_{\mathscr{T}}\right)+S_{T}\left(\theta_{\mathscr{T}}, \psi_{\mathscr{T}}\right)\right| & \leq \tilde{C}_{\mathcal{A}_{T}}\left\|\theta_{\mathscr{T}}\right\|_{\mathcal{T}(\mathscr{T})}\left\|\psi_{\mathscr{T}}\right\|_{\mathcal{T}(\mathscr{T})}, \\
\left.\mid C_{F}\left(\mathscr{L}_{\left(\boldsymbol{\vartheta}_{\mathscr{T}}, \chi\right.}\right) ; \boldsymbol{\xi}_{\mathscr{T}}, \boldsymbol{\zeta}_{\mathscr{T}}\right) \mid & \leq \tilde{C}_{C}\left\|\left(\boldsymbol{\vartheta}_{\mathscr{T}}, \chi_{\mathscr{T}}\right)\right\|_{\mathcal{N}(\mathscr{T})}\left\|\boldsymbol{\xi}_{\mathscr{T}}\right\|_{L^{3}(\Omega)}\left\|\left(\boldsymbol{\zeta}_{\mathscr{T}}, \phi_{\mathscr{T}}\right)\right\|_{\mathcal{N}(\mathscr{T})}, \\
\left|C_{T}\left(\mathscr{L}\left(\boldsymbol{\vartheta}_{\mathscr{T}}, \chi_{\mathscr{T}}\right) ; \theta_{\mathscr{T}}, \psi_{\mathscr{T}}\right)\right| & \leq \tilde{C}_{\mathrm{c}}\left\|\left(\boldsymbol{\vartheta}_{\mathscr{T}}, \chi_{\mathscr{T}}\right)\right\|_{\mathcal{N}(\mathscr{T})}\left\|\theta_{\mathscr{T}}\right\|_{L^{3}(\Omega)}\left\|\psi_{\mathscr{T}}\right\|_{\mathcal{T}(\mathscr{T})} . \\
\left|C_{F}\left(\mathscr{L}(\boldsymbol{\vartheta}, \chi) ; \boldsymbol{\xi}, \boldsymbol{\zeta}_{\mathscr{T}}\right)\right| & \leq \tilde{C}_{C 2}\|(\boldsymbol{\vartheta}, \chi)\|_{\mathcal{N}(\mathscr{T})}\|\boldsymbol{\xi}\|_{W^{1, \infty}(\Omega)}\left\|\left(\boldsymbol{\zeta}_{\mathscr{T}}, \phi_{\mathscr{T}}\right)\right\|_{\mathcal{N}(\mathscr{T})}, \\
\left|C_{T}\left(\mathscr{L}(\boldsymbol{\vartheta}, \chi) ; \theta, \psi_{\mathscr{T}}\right)\right| & \leq \tilde{C}_{\mathrm{c} 2}\|(\boldsymbol{\vartheta}, \chi)\|_{\mathcal{N}(\mathscr{T})}\|\theta\|_{W^{1, \infty}(\Omega)}\left\|\psi_{\mathscr{T}}\right\|_{\mathcal{T}(\mathscr{T})} .
\end{aligned}
$$

where $\tilde{C}_{g}, \tilde{C}_{\mathcal{A}_{T}}, \tilde{C}_{C}, \tilde{C}_{c}, \tilde{C}_{C 2}, \tilde{C}_{\mathrm{c} 2}$ are positive constants. It also holds, for any $\psi_{1}, \psi_{2} \in H^{1}(\Omega)$, that

$$
\begin{aligned}
& \left|\mathcal{A}_{F}\left(\psi_{1} ; \boldsymbol{\xi}, \boldsymbol{\zeta}_{\mathscr{T}}\right)-\mathcal{A}_{F}\left(\psi_{2} ; \boldsymbol{\xi}, \boldsymbol{\zeta}_{\mathscr{T}}\right)\right| \leq C_{\varepsilon} C_{\varepsilon, l i p}\left\|\psi_{1}-\psi_{2}\right\|_{\mathcal{T}(\mathscr{T})}\|\boldsymbol{\xi}\|_{\boldsymbol{W}^{1, \infty}(\Omega)}\left\|\left(\boldsymbol{\zeta}_{\mathscr{T}}, \phi_{\mathscr{T}}\right)\right\|_{\mathcal{N}(\mathscr{T})}, \\
& \left|\mathcal{A}_{T}\left(\psi_{1} ; \theta, \psi_{\mathscr{T}}\right)-\mathcal{A}_{T}\left(\psi_{2} ; \theta, \psi_{\mathscr{T}}\right)\right| \leq C_{\kappa} C_{\kappa, l i p}\left\|\psi_{1}-\psi_{2}\right\|_{\mathcal{T}(\mathscr{T})}\|\theta\|_{W^{1, \infty}(\Omega)}\left\|\psi_{\mathscr{T}}\right\|_{\mathcal{T}(\mathscr{T})},
\end{aligned}
$$

where $C_{\varepsilon}, C_{\kappa}$ are positive constants. Moreover, the following coercivity properties holds, for all $\iota_{\mathscr{T}} \in V_{0}(\mathscr{T})$,

$$
\begin{gathered}
\mathcal{A}_{F}\left(\psi ; \zeta_{\mathscr{T}}, \zeta_{\mathscr{T}}\right)+S_{F}\left(\boldsymbol{\zeta}_{\mathscr{T}}, \zeta_{\mathscr{T}}\right)+\sum_{\gamma \in \mathcal{F}_{I}} \tau_{\gamma} \int_{\gamma} \llbracket \phi_{\mathscr{T}}\left\|^{2} \geq\right\|\left(\boldsymbol{\zeta}_{\mathscr{T}}, \phi_{\mathscr{T}}\right) \|_{\mathcal{N}(\mathscr{T})}^{2}, \\
\mathcal{A}_{T}\left(\psi ; \iota_{\mathscr{T}}, \iota_{\mathscr{T}}\right)+S_{T}\left(\iota_{\mathscr{T}}, \iota_{\mathscr{T}}\right) \geq \tilde{C}_{c o, T}\left\|\iota_{\mathscr{T}}\right\|_{\mathcal{T}(\mathscr{T})}^{2},
\end{gathered}
$$

where $\tilde{C}_{c o, T}$ is a positive constant.

Proof. See the Appendix. 


\subsection{Existence of a discrete solution}

We start this section with an a priori estimate for any solution of (15). The existence of solutions of (15) that, in addition, satisfy the a priori estimate (35), will be given in Theorem 2 below.

Lemma 5. Let $\left(\mathbf{u}_{\mathscr{T}}, \mathrm{p}_{\mathscr{T}}, \mathrm{t}_{\mathscr{T}}\right)$ be a solution of (15). Let us write $\mathrm{t}_{\mathscr{T}}=\mathrm{t}_{\mathscr{T}, 0}+\mathrm{t}_{\mathscr{T}, 1}$, where $\mathrm{t}_{\mathscr{T}, 0} \in V_{0}(\mathscr{T})$ and $\mathrm{t}_{\mathscr{T}, 1} \in V(\mathscr{T})$ is such that $\left.\mathrm{t}_{\mathscr{T}, 1}\right|_{\Gamma}=i_{h}\left(\mathrm{t}_{D}\right)$. In addition, let us suppose that

$$
\tilde{C}\|\mathbf{g}\|_{L^{2}(\Omega)}\left\|t_{\mathscr{T}, 1}\right\|_{L^{3}(\Omega)} \leq \frac{1}{2} \quad \text { with } \quad \tilde{C}=\frac{\tilde{C}_{g} \tilde{C}_{c}}{\tilde{C}_{c o, T}} .
$$

Then, there exist positive constants $C_{F}$ and $C_{T}$ only depending on $\|\mathbf{g}\|_{L^{2}(\Omega)}$ and the constants defined in Section 4.1 such that

$$
\left\|\left(\mathbf{u}_{\mathscr{T}}, \mathrm{p}_{\mathscr{T}}\right)\right\|_{\mathcal{N}(\mathscr{T})} \leq C_{F}\left\|\mathrm{t}_{\mathscr{T}, 1}\right\|_{\mathcal{T}(\mathscr{T})}, \quad\left\|\mathrm{t}_{\mathscr{T}}\right\|_{\mathcal{T}(\mathscr{T})} \leq C_{T}\left\|\mathrm{t}_{\mathscr{T}, 1}\right\|_{\mathcal{T}(\mathscr{T})} .
$$

Proof. Considering as test function $\left(\boldsymbol{\xi}_{\mathscr{T}}, \phi_{\mathscr{T}}, \psi_{\mathscr{T}}\right)=\left(\mathbf{u}_{\mathscr{T}}, \mathrm{p}_{\mathscr{T}}, \mathrm{t}_{\mathscr{T}, 0}\right)$ in (15) and adding the first two equations gives

$$
\begin{aligned}
\mathcal{A}_{F}\left(\mathrm{t}_{\mathscr{T}} ; \mathbf{u}_{\mathscr{T}}, \mathbf{u}_{\mathscr{T}}\right)+\mathcal{C}_{F}\left(\mathscr{L}\left(\mathbf{u}_{\mathscr{T}}, \mathrm{p}_{\mathscr{T}}\right) ; \mathbf{u}_{\mathscr{T}}, \mathbf{u}_{\mathscr{T}}\right)+\mathcal{S}_{F}\left(\mathbf{u}_{\mathscr{T}}, \mathbf{u}_{\mathscr{T}}\right)+\sum_{\gamma \in \mathscr{F}_{I}} \tau_{\gamma} \int_{\gamma} \llbracket \mathrm{p}_{\mathscr{T}} \mathbb{1}_{\gamma}^{2}=\mathcal{G}_{F}\left(\mathrm{t}_{\mathscr{T}}, \mathbf{u}_{\mathscr{T}}\right), \\
\mathcal{A}_{T}\left(\mathrm{t}_{\mathscr{T}} ; \mathrm{t}_{\mathscr{T}}, \mathrm{t}_{\mathscr{T}, 0}\right)+\mathcal{C}_{T}\left(\mathscr{L}\left(\mathbf{u}_{\mathscr{T}}, \mathrm{p}_{\mathscr{T}}\right) ; \mathrm{t}_{\mathscr{T}}, \mathrm{t}_{\mathscr{T}, 0}\right)+\mathcal{S}_{T}\left(\mathrm{t}_{\mathscr{T}}, \mathrm{t}_{\mathscr{T}, 0}\right)=0 .
\end{aligned}
$$

Next, expressing $\mathrm{t}_{\mathscr{T}}$ as $\mathrm{t}_{\mathscr{T}}=\mathrm{t}_{\mathscr{T}, 0}+\mathrm{t}_{\mathscr{T}, 1}$ and using (12) and (14) we obtain

$$
\begin{aligned}
& \mathcal{A}_{F}\left(\mathrm{t}_{\mathscr{T}} ; \mathbf{u}_{\mathscr{T}}, \mathbf{u}_{\mathscr{T}}\right)+\mathcal{S}_{F}\left(\mathbf{u}_{\mathscr{T}}, \mathbf{u}_{\mathscr{T}}\right)+\sum_{\gamma \in \mathscr{F}_{I}} \tau_{\gamma} \int_{\gamma} \llbracket \mathrm{p}_{\mathscr{T}} \rrbracket_{\gamma}^{2}=\mathcal{G}_{F}\left(\mathrm{t}_{\mathscr{T}, 0}, \mathbf{u}_{\mathscr{T}}\right)+\mathcal{G}_{F}\left(\mathrm{t}_{\mathscr{T}, 1}, \mathbf{u}_{\mathscr{T}}\right), \\
& \mathcal{A}_{T}\left(\mathrm{t}_{\mathscr{T}} ; \mathrm{t}_{\mathscr{T}, 0}, \mathrm{t}_{\mathscr{T}, 0}\right)+\mathcal{S}_{T}\left(\mathrm{t}_{\mathscr{T}, 0}, \mathrm{t}_{\mathscr{T}, 0}\right)=-\mathcal{A}_{T}\left(\mathrm{t}_{\mathscr{T}} ; \mathrm{t}_{\mathscr{T}, 1}, \mathrm{t}_{\mathscr{T}, 0}\right)-C_{T}\left(\mathscr{L}\left(\mathbf{u}_{\mathscr{T}}, \mathrm{p}_{\mathscr{T}}\right) ; \mathrm{t}_{\mathscr{T}, 1}, \mathrm{t}_{\mathscr{T}, 0}\right)-\mathcal{S}_{T}\left(\mathrm{t}_{\mathscr{T}, 1}, \mathrm{t}_{\mathscr{T}, 0}\right) .
\end{aligned}
$$

Using, in the first equation of (36), (32) and (24), and dividing through by $\left\|\left(\mathbf{u}_{\mathscr{T}}, \mathrm{p}_{\mathscr{T}}\right)\right\|_{\mathcal{N}(\mathscr{T})}$ we arrive at

$$
\left\|\left(\mathbf{u}_{\mathscr{T}}, \mathrm{p}_{\mathscr{T}}\right)\right\|_{\mathcal{N}(\mathscr{T})} \leq \tilde{C}_{g}\|\mathbf{g}\|_{L^{2}(\Omega)}\left(\left\|\mathrm{t}_{\mathscr{T}, 0}\right\|_{\mathcal{T}(\mathscr{T})}+\left\|\mathrm{t}_{\mathscr{T}, 1}\right\|_{\mathcal{T}(\mathscr{T})}\right) .
$$

Proceeding analogously on the second equation of (36), i.e., using (33), (25), (27), and next simplifying the term $\tilde{C}_{c o, T}\left\|t_{\mathscr{T}, 0}\right\|_{\mathcal{T}(\mathscr{T})}$, we get

$$
\left\|\mathrm{t}_{\mathscr{T}, 0}\right\|_{\mathcal{T}(\mathscr{T})} \leq \tilde{C}_{c o, T}^{-1}\left(\tilde{C}_{\mathcal{H}_{T}}\left\|\mathrm{t}_{\mathscr{T}, 1}\right\|_{\mathcal{T}(\mathscr{T})}+\tilde{C}_{\mathrm{c}}\left\|\left(\mathbf{u}_{\mathscr{T}}, \mathrm{p}_{\mathscr{T}}\right)\right\|_{\mathcal{N}(\mathscr{T})}\left\|\mathrm{t}_{\mathscr{T}, 1}\right\|_{L^{3}(\Omega)}\right) .
$$

Replacing (38) in (37), and reordering terms, it follows that

$$
\begin{aligned}
& \left\|\left(\mathbf{u}_{\mathscr{T}}, \mathrm{p}_{\mathscr{T}}\right)\right\|_{\mathcal{N}(\mathscr{T})} \\
& \leq \tilde{C}_{g}\|\mathbf{g}\|_{L^{2}(\Omega)}\left(\tilde{C}_{c o, T}^{-1} \tilde{C}_{\mathcal{F}_{T}}\left\|\mathrm{t}_{\mathscr{T}, 1}\right\|_{\mathcal{T}(\mathscr{T})}+\tilde{C}_{c o, T}^{-1} \tilde{C}_{c}\left\|\left(\mathbf{u}_{\mathscr{T}}, \mathrm{p}_{\mathscr{T}}\right)\right\|_{\mathcal{N}(\mathscr{T})}\left\|\mathrm{t}_{\mathscr{T}, 1}\right\|_{L^{3}(\Omega)}+\left\|\mathrm{t}_{\mathscr{T}, 1}\right\|_{\mathcal{T}(\mathscr{T})}\right) \\
& =\tilde{C}_{g}\|\mathbf{g}\|_{L^{2}(\Omega)}\left(\tilde{C}_{c o, T}^{-1} \tilde{C}_{\mathcal{F}_{T}}+1\right)\left\|\mathrm{t}_{\mathscr{T}, 1}\right\|_{\mathcal{T}(\mathscr{T})}+\tilde{C}_{c o, T}^{-1} \tilde{C}_{g} \tilde{C}_{\mathrm{c}}\|\mathbf{g}\|_{L^{2}(\Omega)}\left\|\left(\mathbf{u}_{\mathscr{T}}, \mathrm{p}_{\mathscr{T}}\right)\right\|_{\mathcal{N}(\mathscr{T})}\left\|\mathrm{t}_{\mathscr{T}, 1}\right\|_{L^{3}(\Omega)}
\end{aligned}
$$

Therefore, using assumption (34) we conclude that

$$
\frac{1}{2}\left\|\left(\mathbf{u}_{\mathscr{T}}, \mathrm{p}_{\mathscr{T}}\right)\right\|_{\mathcal{N}(\mathscr{T})} \leq \tilde{C}_{g}\|\mathbf{g}\|_{L^{2}(\Omega)}\left(\tilde{C}_{c o, T}^{-1} \tilde{C}_{\mathcal{H}_{T}}+1\right)\left\|\mathrm{t}_{\mathscr{T}, 1}\right\|_{\mathcal{T}(\mathscr{T})} .
$$

Thus, we have proved that

$$
\left\|\left(\mathbf{u}_{\mathscr{T}}, \mathrm{p}_{\mathscr{T}}\right)\right\|_{\mathcal{N}(\mathscr{T})} \leq C_{F}\left\|\mathrm{t}_{\mathscr{T}, 1}\right\|_{\mathcal{T}(\mathscr{T})} \quad \text { with } \quad C_{F}=2 \tilde{C}_{c o, T}^{-1} \tilde{C}_{g}\|\mathbf{g}\|_{L^{2}(\Omega)}\left(\tilde{C}_{\mathcal{H}_{T}}+\tilde{C}_{c o, T}\right) .
$$


On the other hand, using the triangle inequality, (38), (39), reordering terms and considering the assumption given in (34), we obtain that

$$
\begin{aligned}
& \left\|\mathrm{t}_{\mathscr{T}}\right\|_{\mathcal{T}(\mathscr{T})} \\
& \leq\left\|\mathrm{t}_{\mathscr{T}, 0}\right\|_{\mathcal{T}(\mathscr{T})}+\left\|\mathrm{t}_{\mathscr{T}, 1}\right\|_{\mathcal{T}(\mathscr{T})} \\
& \leq\left(\tilde{C}_{c o, T}^{-1} \tilde{C}_{\mathcal{A}_{T}}+1\right)\left\|\mathrm{t}_{\mathscr{T}, 1}\right\|_{\mathcal{T}(\mathscr{T})}+\tilde{C}_{c o, T}^{-1} \tilde{C}_{\mathrm{c}}\left\|\left(\mathbf{u}_{\mathscr{T}}, \mathrm{p}_{\mathscr{T}}\right)\right\|_{\mathcal{N}(\mathscr{T})}\left\|\mathrm{t}_{\mathscr{T}, 1}\right\|_{L^{3}(\Omega)} \\
& \leq\left(\tilde{C}_{c o, T}^{-1} \tilde{C}_{\mathcal{A}_{T}}+1\right)\left\|\mathrm{t}_{\mathscr{T}, 1}\right\|_{\mathcal{T}(\mathscr{T})}+\tilde{C}_{c o, T}^{-1} \tilde{C}_{\mathrm{c}} 2 \tilde{C}_{c o, T}^{-1} \tilde{C}_{g}\|\mathbf{g}\|_{L^{2}(\Omega)}\left(\tilde{C}_{\mathcal{A}_{T}}+\tilde{C}_{c o, T}\right)\left\|\mathrm{t}_{\mathscr{T}, 1}\right\|_{\mathcal{T}(\mathscr{T})}\left\|\mathrm{t}_{\mathscr{T}, 1}\right\|_{L^{3}(\Omega)} \\
& =\left(\tilde{C}_{c o, T}^{-1} \tilde{C}_{\mathcal{A}_{T}}+1\right)\left\|\mathrm{t}_{\mathscr{T}, 1}\right\|_{\mathcal{T}(\mathscr{T})}+2 \tilde{C}_{c o, T}^{-1}\left(\tilde{C}_{\mathcal{A}_{T}}+\tilde{C}_{c o, T}\right)\left(\frac{\tilde{C}_{g} \tilde{C}_{c}}{\tilde{C}_{c o, T}}\|\mathbf{g}\|_{L^{2}(\Omega)}\left\|\mathrm{t}_{\mathscr{T}, 1}\right\|_{L^{3}(\Omega)}\right)\left\|\mathrm{t}_{\mathscr{T}, 1}\right\|_{\mathcal{T}(\mathscr{T})} \\
& \leq\left(\tilde{C}_{c o, T}^{-1} \tilde{C}_{\mathcal{A}_{T}}+1\right)\left\|\mathrm{t}_{\mathscr{T}, 1}\right\|_{\mathcal{T}(\mathscr{T})}+\tilde{C}_{c o, T}^{-1}\left(\tilde{C}_{\mathcal{A}_{T}}+\tilde{C}_{c o, T}\right)\left\|\mathrm{t}_{\mathscr{T}, 1}\right\|_{\mathcal{T}(\mathscr{T})} .
\end{aligned}
$$

Therefore,

$$
\left\|\mathrm{t}_{\mathscr{T}}\right\|_{\mathcal{T}(\mathscr{T})} \leq C_{T}\left\|_{\mathscr{T}, 1}\right\|_{\mathcal{T}(\mathscr{T})} \quad \text { with } \quad C_{T}=2 \tilde{C}_{c o, T}^{-1}\left(\tilde{C}_{\mathcal{H}_{T}}+\tilde{C}_{c o, T}\right)
$$

This completes the proof.

Remark 4. Since the lift $\mathrm{t}_{\mathscr{T}, 1}$ needs to have a $L^{3}(\Omega)$-norm small enough, its existence can be shown for a mesh that is refined enough around the boundary. For an in depth discussion of this, and other alternatives to build $\mathrm{t}_{\mathscr{T}, 1}$, see [15, Section 4].

Theorem 2. Let us assume all hypotheses of Lemma 5. Then there exists $\left(\mathbf{u}_{\mathscr{T}}, \mathrm{p}_{\mathscr{T}}, \mathrm{t}_{\mathscr{T}}\right) \in \boldsymbol{V}_{0}(\mathscr{T}) \times Q(\mathscr{T}) \times V(\mathscr{T})$ solution of (15).

Proof. To prove the result, we will use Brouwer's fixed point Theorem. We recall that we fix the lift $\mathrm{t}_{\mathscr{T}, 1}$ satisfying (34). Therefore, we define the following mapping:

$$
\begin{array}{cccc}
\mathbb{T}: & \tilde{M} & \rightarrow & \boldsymbol{V}_{0}(\mathscr{T}) \times Q(\mathscr{T}) \times V(\mathscr{T}) \\
\left(\zeta_{\mathscr{T}}, \chi \mathscr{T}, \theta_{\mathscr{T}}\right) & \mapsto & \mathbb{T}\left(\zeta_{\mathscr{T}}, \chi \mathscr{T}, \theta \mathscr{T}\right)=\left(\mathbf{u}_{\mathscr{T}}, \mathrm{p}_{\mathscr{T}}, \mathrm{t}_{\mathscr{T}}\right)
\end{array}
$$

where the vector space $\tilde{M}$ is defined as

$$
\tilde{M}=\left\{\left(\zeta_{\mathscr{T}}, \chi_{\mathscr{T}}, \theta_{\mathscr{T}}\right) \in \boldsymbol{V}_{0}(\mathscr{T}) \times Q(\mathscr{T}) \times V(\mathscr{T}): \operatorname{div} \mathscr{L}\left(\zeta_{\mathscr{T}}, \chi \mathscr{T}\right)=0\right\}
$$

and $\left(\mathbf{u}_{\mathscr{T}}, \mathrm{p}_{\mathscr{T}}, \mathrm{t}_{\mathscr{T}}\right)$ is the solution to

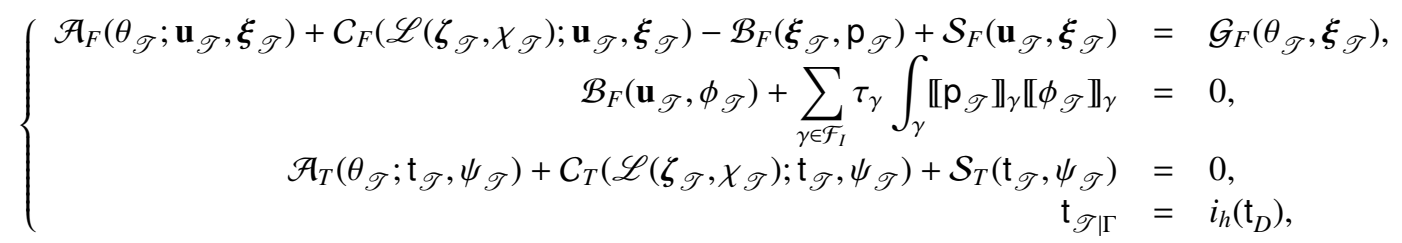

for all $\left(\boldsymbol{\xi}_{\mathscr{T}}, \phi_{\mathscr{T}}, \psi_{\mathscr{T}}\right) \in \boldsymbol{V}_{0}(\mathscr{T}) \times Q(\mathscr{T}) \times V_{0}(\mathscr{T})$. Also we define the convex, bounded, and closed set $M$ given by

$$
M=\left\{\left(\zeta_{\mathscr{T}}, \chi_{\mathscr{T}}, \theta_{\mathscr{T}}\right) \in \tilde{M}:\left\|\left(\zeta_{\mathscr{T}}, \chi_{\mathscr{T}}\right)\right\|_{\mathcal{N}(\mathscr{T})} \leq C_{F}\left\|\mathrm{t}_{\mathscr{T}, 1}\right\|_{\mathcal{T}(\mathscr{T})}, \quad\left\|\theta_{\mathscr{T}}\right\|_{\mathcal{T}(\mathscr{T})} \leq C_{T}\left\|_{\mathrm{T}_{, 1}}\right\|_{\mathcal{T}(\mathscr{T})}\right\}
$$

where $C_{F}$ and $C_{T}$ are given in (39) and (40), respectively. With the purpose of using Brouwer's fixed point Theorem, we will divide the proof into 3 steps.

Step 1, $\mathbb{T}$ is well-defined: First, notice that (41) is a decoupled problem. The first two equations correspond to a stabilized finite element formulation of an Oseen equation (related to the one presented, e.g., in [28]), with a convective term $\mathcal{L}\left(\zeta_{\mathscr{T}}, \chi_{\mathscr{T}}\right)$ that is solenoidal for any $\left(\boldsymbol{\zeta}_{\mathscr{T}}, \chi_{\mathscr{T}}, \theta_{\mathscr{T}}\right) \in \tilde{M}$, which, as has been shown in [28], has a unique solution due to all the assumptions previously stated for $\varepsilon$ and $\mathcal{S}_{F}$. Similarly, the last equation of (41) corresponds to a stabilized finite element formulation of a convection-diffusion equation that is well posed based on 
the restrictions on $\kappa, \mathcal{S}_{T}$ and the fact that $\mathcal{L}\left(\zeta_{\mathscr{T}}, \chi_{\mathscr{T}}\right)$ is solenoidal for any $\left(\boldsymbol{\zeta}_{\mathscr{T}}, \chi_{\mathscr{T}}, \theta_{\mathscr{T}}\right) \in \tilde{M}$ (see, e.g., [29] for a detailed analysis for the case of a CIP method).

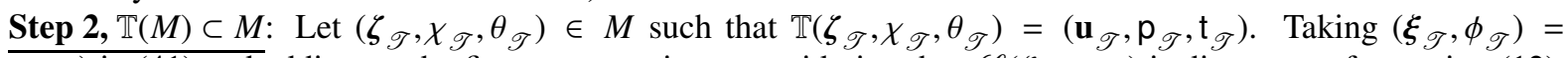
$\left(\mathbf{u}_{\mathscr{T}}, \mathrm{p}_{\mathscr{T}}\right)$ in (41) and adding up the first two equations, considering that $\mathscr{L}\left(\zeta_{\mathscr{T}}, \chi \mathscr{T}\right)$ is divergence-free, using (12), and the properties given in (32) and (24), it follows that

$$
\left\|\left(\mathbf{u}_{\mathscr{T}}, \mathbf{p}_{\mathscr{T}}\right)\right\|_{\mathcal{N}(\mathscr{T})}^{2} \leq \tilde{C}_{g}\|\mathbf{g}\|_{L^{2}(\Omega)}\left\|\left(\mathbf{u}_{\mathscr{T}}, \mathbf{p}_{\mathscr{T}}\right)\right\|_{\mathcal{N}(\mathscr{T})}\left\|\theta_{\mathscr{T}}\right\|_{\mathcal{T}(\mathscr{T})}
$$

Considering that $\left(\zeta_{\mathscr{T}}, \chi_{\mathscr{T}}, \theta_{\mathscr{T}}\right) \in M$ and the definition of the constant $C_{T}$ in (40) we get

$$
\left\|\left(\mathbf{u}_{\mathscr{T}}, \mathrm{p}_{\mathscr{T}}\right)\right\|_{\mathcal{N}(\mathscr{T})} \leq \tilde{C}_{g} 2 \tilde{C}_{c o, T}^{-1}\left(\tilde{C}_{\mathcal{A}_{T}}+\tilde{C}_{c o, T}\right)\|\mathbf{g}\|_{L^{2}(\Omega)}\left\|\mathbf{t}_{\mathscr{T}, 1}\right\|_{\mathcal{T}(\mathscr{T})} .
$$

Therefore, from the definition of the constant $C_{F}$ in (39), we obtain that $\left\|\left(\mathbf{u}_{\mathscr{T}}, \mathrm{p}_{\mathscr{T}}\right)\right\|_{\mathcal{N}(\mathscr{T})} \leq C_{F}\left\|_{\mathfrak{T}_{1}, 1}\right\|_{\mathcal{T}(\mathscr{T})}$.

On the other hand, taking $\psi_{\mathscr{T}}=\mathrm{t}_{\mathscr{T}, 0}$ in (41), writing $\mathrm{t}_{\mathscr{T}}$ as $\mathrm{t}_{\mathscr{T}}=\mathrm{t}_{\mathscr{T}, 0}+\mathrm{t}_{\mathscr{T}, 1}$ and reordering terms, it follows that

$$
\begin{aligned}
& \mathcal{A}_{T}\left(\theta_{\mathscr{T}} ; \mathrm{t}_{\mathscr{T}, 0}, \mathrm{t}_{\mathscr{T}, 0}\right)+\mathcal{C}_{T}\left(\mathscr{L}\left(\zeta_{\mathscr{T}}, \chi \mathscr{T}\right) ; \mathrm{t}_{\mathscr{T}, 0}, \mathrm{t}_{\mathscr{T}, 0}\right)+\mathcal{S}_{T}\left(\mathrm{t}_{\mathscr{T}, 0}, \mathrm{t}_{\mathscr{T}, 0}\right) \\
& \leq-\mathcal{A}_{T}\left(\theta \mathscr{T} ; \mathrm{t}_{\mathscr{T}, 1}, \mathrm{t}_{\mathscr{T}, 0}\right)-\mathcal{C}_{T}\left(\mathscr{L}_{(}\left(\boldsymbol{\zeta}_{\mathscr{T}}, \chi \mathscr{T}\right) ; \mathrm{t}_{\mathscr{T}, 1}, \mathrm{t}_{\mathscr{T}, 0}\right)-\mathcal{S}_{T}\left(\mathrm{t}_{\mathscr{T}, 1}, \mathrm{t}_{\mathscr{T}, 0}\right) .
\end{aligned}
$$

On the left hand side on the last inequality we use (33) and (14). On the right hand side we bound each term using (25), (27), and the fact that $\left(\zeta_{\mathscr{T}}, \chi_{\mathscr{T}}, \theta_{\mathscr{T}}\right) \in M$. We thus get the following bound

$$
\begin{aligned}
\tilde{C}_{c o, T}\left\|\mathrm{t}_{\mathscr{T}, 0}\right\|_{\mathcal{T}_{(\mathscr{T})}}^{2} & \leq\left|\mathcal{A}_{T}\left(\theta_{\mathscr{T}} ; \mathrm{t}_{\mathscr{T}, 1}, \mathrm{t}_{\mathscr{T}, 0}\right)+\mathcal{S}_{T}\left(\mathrm{t}_{\mathscr{T}, 1}, \mathrm{t}_{\mathscr{T}, 0}\right)\right|+\mid \mathcal{C}_{T}\left(\mathscr{L}\left(\zeta_{\mathscr{T}}, \chi \mathscr{T}\right) ; \mathrm{t}_{\mathscr{T}, 1}, \mathrm{t}_{\mathscr{T}, 0} \mid\right. \\
& \leq \tilde{C}_{\mathcal{F}_{T}}\left\|\mathrm{t}_{\mathscr{T}, 1}\right\|_{\mathcal{T}(\mathscr{T})}\left\|\mathrm{t}_{\mathscr{T}, 0}\right\|_{\mathcal{T}(\mathscr{T})}+\tilde{C}_{\mathrm{c}}\left\|\left(\zeta_{\mathscr{T}}, \chi \mathscr{T}\right)\right\|_{\mathcal{N}(\mathscr{T})}\left\|\mathrm{t}_{\mathscr{T}, 0}\right\|_{\mathcal{T}(\mathscr{T})}\left\|\mathrm{t}_{\mathscr{T}, 1}\right\|_{L^{3}(\Omega)} \\
& \leq \tilde{C}_{\mathcal{F}_{T}}\left\|\mathrm{t}_{\mathscr{T}, 1}\right\|_{\mathcal{T}(\mathscr{T})}\left\|\mathrm{t}_{\mathscr{T}, 0}\right\|_{\mathcal{T}(\mathscr{T})}+\tilde{C}_{\mathrm{c}} C_{F}\left\|\mathrm{t}_{\mathscr{T}, 1}\right\|_{\mathcal{T}(\mathscr{T})}\left\|\mathrm{t}_{\mathscr{T}, 0}\right\|_{\mathcal{T}(\mathscr{T})}\left\|\mathrm{t}_{\mathscr{T}, 1}\right\|_{L^{3}(\Omega)}
\end{aligned}
$$

Next, we simplify the term $\left\|\mathrm{t}_{\mathscr{T}, 0}\right\|_{\mathcal{T}(\mathscr{T})}$, reorder terms, use the definition of the constant $C_{F}$ in (39), and (34) to obtain

$$
\begin{aligned}
\tilde{C}_{c o, T}\left\|\mathrm{t}_{\mathscr{T}, 0}\right\|_{\mathcal{T}(\mathscr{T})} & \leq\left(\tilde{C}_{\mathcal{A}_{T}}+\tilde{C}_{\mathrm{c}} C_{F}\left\|\mathrm{t}_{\mathscr{T}, 1}\right\|_{L^{3}(\Omega)}\right)\left\|\mathrm{t}_{\mathscr{T}, 1}\right\|_{\mathcal{T}(\mathscr{T})} \\
& =\left(\tilde{C}_{\mathcal{H}_{T}}+\tilde{C}_{\mathrm{c}} 2 \tilde{C}_{c o, T}^{-1} \tilde{C}_{g}\|\mathbf{g}\|_{L^{2}(\Omega)}\left(\tilde{C}_{\mathcal{A}_{T}}+\tilde{C}_{c o, T}\right)\left\|\mathrm{t}_{\mathscr{T}, 1}\right\|_{L^{3}(\Omega)}\right)\left\|\mathrm{t}_{\mathscr{T}, 1}\right\|_{\mathcal{T}(\mathscr{T})} \\
& \leq\left(\tilde{C}_{\mathcal{F}_{T}}+2\left(\tilde{C}_{\mathcal{H}_{T}}+\tilde{C}_{c o, T}\right)\left(\frac{\tilde{C}_{c} \tilde{C}_{g}}{\tilde{C}_{c o, T}}\|\mathbf{g}\|_{L^{2}(\Omega)}\left\|\mathrm{t}_{\mathscr{T}, 1}\right\|_{L^{3}(\Omega)}\right)\right)\left\|\mathrm{t}_{\mathscr{T}, 1}\right\|_{\mathcal{T}(\mathscr{T})} \\
& \leq\left(\tilde{C}_{\mathcal{H}_{T}}+\left(\tilde{C}_{\mathcal{A}_{T}}+\tilde{C}_{c o, T}\right)\right)\left\|\mathrm{t}_{\mathscr{T}, 1}\right\|_{\mathcal{T}(\mathscr{T})} .
\end{aligned}
$$

Hence,

$$
\left\|\mathrm{t}_{\mathscr{T}, 0}\right\|_{\mathcal{T}(\mathscr{T})} \leq\left(2 \tilde{C}_{c o, T}^{-1} \tilde{C}_{\mathcal{A}_{T}}+1\right)\left\|\mathrm{t}_{\mathscr{T}, 1}\right\|_{\mathcal{T}(\mathscr{T})} .
$$

Finally, using the triangle inequality, (42) and the constant $C_{T}$ from (40), it follows that

$$
\begin{aligned}
\left\|\mathrm{t}_{\mathscr{T}}\right\|_{\mathcal{T}(\mathscr{T})} \leq\left\|\mathrm{t}_{\mathscr{T}, 0}\right\|_{\mathcal{T}(\mathscr{T})}+\left\|\mathrm{t}_{\mathscr{T}, 1}\right\|_{\mathcal{T}(\mathscr{T})} \leq\left(2 \tilde{C}_{c o, T}^{-1} \tilde{C}_{\mathcal{F}_{T}}+1\right)\left\|\mathrm{t}_{\mathscr{T}, 1}\right\|_{\mathcal{T}(\mathscr{T})}+\left\|\mathrm{t}_{\mathscr{T}, 1}\right\|_{\mathcal{T}(\mathscr{T})} \\
\leq C_{T}\left\|\mathrm{t}_{\mathscr{T}, 1}\right\|_{\mathcal{T}(\mathscr{T}) .}
\end{aligned}
$$

To show that $\left(\mathbf{u}_{\mathscr{T}}, \mathrm{p}_{\mathscr{T}}, \mathrm{t}_{\mathscr{T}}\right) \in M$, it only remains to prove that $\operatorname{div} \mathscr{L}\left(\mathbf{u}_{\mathscr{T}}, \mathrm{p}_{\mathscr{T}}\right)=0$ in $\Omega$, which follows from the fact that $\left(\mathbf{u}_{\mathscr{T}}, \mathrm{p}_{\mathscr{T}}\right)$ satisfies (41), for which the same arguments presented in the proof of Theorem 1 hold.

Step 3, T is continuous: Let $\left\{\left(\boldsymbol{\zeta}_{\mathscr{T}, m}, \chi_{\mathscr{T}, m}, \theta_{\mathscr{T}, m}\right)\right\}_{m} \subseteq \boldsymbol{V}_{0}(\mathscr{T}) \times Q(\mathscr{T}) \times V(\mathscr{T})$ be a sequence converging to $\left(\boldsymbol{\zeta}_{\mathscr{T}}, \chi_{\mathscr{T}}, \theta_{\mathscr{T}}\right) \in \boldsymbol{V}_{0}(\mathscr{T}) \times Q(\mathscr{T}) \times V(\mathscr{T})$ and let $\left\{\left(\mathbf{u}_{\mathscr{T}, m}, \mathrm{p}_{\mathscr{T}, m}, \mathrm{t}_{\mathscr{T}, m}\right)\right\}_{m} \subseteq \boldsymbol{V}_{0}(\mathscr{T}) \times Q(\mathscr{T}) \times V(\mathscr{T})$ and $\left(\mathbf{u}_{\mathscr{T}}, \mathrm{p}_{\mathscr{T}}, \mathrm{t}_{\mathscr{T}}\right) \in$ $\boldsymbol{V}_{0}(\mathscr{T}) \times Q(\mathscr{T}) \times V(\mathscr{T})$ be given by

$$
\mathbb{T}\left(\boldsymbol{\zeta}_{\mathscr{T}, m}, \chi_{\mathscr{T}, m}, \theta_{\mathscr{T}, m}\right)=\left(\mathbf{u}_{\mathscr{T}, m}, \mathbf{p}_{\mathscr{T}, m}, \mathrm{t}_{\mathscr{T}, m}\right) \quad \forall m \in \mathbb{N} \quad \text { and } \quad \mathbb{T}\left(\boldsymbol{\zeta}_{\mathscr{T}}, \chi_{\mathscr{T}}, \theta_{\mathscr{T}}\right)=\left(\mathbf{u}_{\mathscr{T}}, \mathbf{p}_{\mathscr{T}}, \mathrm{t}_{\mathscr{T}}\right)
$$

We will show that $\left(\mathbf{u}_{\mathscr{T}, m}, \mathbf{p}_{\mathscr{T}, m}, \mathbf{t}_{\mathscr{T}, m}\right) \stackrel{m \rightarrow \infty}{\longrightarrow}\left(\mathbf{u}_{\mathscr{T}}, \mathbf{p}_{\mathscr{T}}, \mathbf{t}_{\mathscr{T}}\right)$. Letting $m \in \mathbb{N}$, substracting the equations appearing in (43), considering (41) taking as test functions $\boldsymbol{\xi}_{\mathscr{T}}=\mathbf{u}_{\mathscr{T}}-\mathbf{u}_{\mathscr{T}, m}$ and $\phi_{\mathscr{T}}=\mathrm{p}_{\mathscr{T}}-\mathrm{p}_{\mathscr{T}, m}$, we get

$$
\begin{aligned}
& \left.\mathcal{A}_{F}\left(\theta \mathscr{T} ; \mathbf{u}_{\mathscr{T}}, \mathbf{u}_{\mathscr{T}}-\mathbf{u}_{\mathscr{T}, m}\right)-\mathcal{A}_{F}\left(\theta_{\mathscr{T}, m} ; \mathbf{u}_{\mathscr{T}, m}, \mathbf{u}_{\mathscr{T}}-\mathbf{u}_{\mathscr{T}, m}\right)+\mathcal{C}_{F}\left(\mathscr{L}_{\left(\zeta_{\mathscr{T}}, \chi \mathscr{T}\right.}\right) ; \mathbf{u}_{\mathscr{T}}, \mathbf{u}_{\mathscr{T}}-\mathbf{u}_{\mathscr{T}, m}\right) \\
& -\mathcal{C}_{F}\left(\mathscr{L}\left(\boldsymbol{\zeta}_{\mathscr{T}, m}, \chi \mathscr{T}, m\right) ; \mathbf{u}_{\mathscr{T}, m}, \mathbf{u}_{\mathscr{T}}-\mathbf{u}_{\mathscr{T}, m}\right)+\mathcal{S}_{F}\left(\mathbf{u}_{\mathscr{T}}-\mathbf{u}_{\mathscr{T}, m}, \mathbf{u}_{\mathscr{T}}-\mathbf{u}_{\mathscr{T}, m}\right) \\
& +\sum_{\gamma \in \mathscr{T}_{I}} \tau_{\gamma} \int_{\gamma} \llbracket \mathrm{p}_{\mathscr{T}}-\mathrm{p}_{\mathscr{T}, m} \rrbracket_{\gamma} \llbracket \mathbf{p}_{\mathscr{T}}-\mathrm{p}_{\mathscr{T}, m} \rrbracket_{\gamma}=\mathcal{G}_{F}\left(\theta_{\mathscr{T}}-\theta_{\mathscr{T}, m}, \mathbf{u}_{\mathscr{T}}-\mathbf{u}_{\mathscr{T}, m}\right) .
\end{aligned}
$$


We add and subtract the terms $\mathcal{A}_{F}\left(\theta_{\mathscr{T}, m} ; \mathbf{u}_{\mathscr{T}}, \mathbf{u}_{\mathscr{T}}-\mathbf{u}_{\mathscr{T}, m}\right)$ and $\mathcal{C}_{F}\left(\mathscr{L}\left(\boldsymbol{\zeta}_{\mathscr{T}, m}, \chi \mathscr{T}, m\right) ; \mathbf{u}_{\mathscr{T}}, \mathbf{u}_{\mathscr{T}}-\mathbf{u}_{\mathscr{T}, m}\right)$, we consider the fact that $\mathscr{L}(\cdot)$ is linear and the definition of $\mathcal{A}_{F}$ given in (10) to obtain

$$
\begin{aligned}
& \mathcal{A}_{F}\left(\theta_{\mathscr{T}} ; \mathbf{u}_{\mathscr{T}}, \mathbf{u}_{\mathscr{T}}-\mathbf{u}_{\mathscr{T}, m}\right)-\mathcal{A}_{F}\left(\theta_{\mathscr{T}, m} ; \mathbf{u}_{\mathscr{T}}, \mathbf{u}_{\mathscr{T}}-\mathbf{u}_{\mathscr{T}, m}\right)+\mathcal{A}_{F}\left(\theta_{\mathscr{T}, m} ; \mathbf{u}_{\mathscr{T}}-\mathbf{u}_{\mathscr{T}, m}, \mathbf{u}_{\mathscr{T}}-\mathbf{u}_{\mathscr{T}, m}\right) \\
& \left.\left.+\mathcal{C}_{F}\left(\mathscr{L}_{\left(\zeta_{\mathscr{T}}\right.}-\boldsymbol{\zeta}_{\mathscr{T}, m}, \chi \mathscr{T}-\chi \mathcal{T}_{, m}\right) ; \mathbf{u}_{\mathscr{T}}, \mathbf{u}_{\mathscr{T}}-\mathbf{u}_{\mathscr{T}, m}\right)+C_{F}\left(\mathscr{L}_{\left(\zeta_{\mathscr{T}, m}, \chi \mathscr{T}, m\right.}\right) ; \mathbf{u}_{\mathscr{T}}-\mathbf{u}_{\mathscr{T}, m}, \mathbf{u}_{\mathscr{T}}-\mathbf{u}_{\mathscr{T}, m}\right) \\
& +\mathcal{S}_{F}\left(\mathbf{u}_{\mathscr{T}}-\mathbf{u}_{\mathscr{T}, m}, \mathbf{u}_{\mathscr{T}}-\mathbf{u}_{\mathscr{T}, m}\right)+\sum_{\gamma \in \mathcal{F}_{I}} \tau_{\gamma} \int_{\gamma} \llbracket \mathrm{p}_{\mathscr{T}}-\mathrm{p}_{\mathscr{T}, m} \rrbracket_{\gamma}^{2}=\mathcal{G}_{F}\left(\theta_{\mathscr{T}}-\theta_{\mathscr{T}, m}, \mathbf{u}_{\mathscr{T}}-\mathbf{u}_{\mathscr{T}, m}\right)
\end{aligned}
$$

Using (12) and reordering terms it follows that

$$
\begin{aligned}
\mathcal{A}_{F}\left(\theta_{\mathscr{T}, m} ; \mathbf{u}_{\mathscr{T}}-\mathbf{u}_{\mathscr{T}, m}, \mathbf{u}_{\mathscr{T}}-\mathbf{u}_{\mathscr{T}, m}\right)+\mathcal{S}_{F}\left(\mathbf{u}_{\mathscr{T}}-\mathbf{u}_{\mathscr{T}, m}, \mathbf{u}_{\mathscr{T}}-\mathbf{u}_{\mathscr{T}, m}\right)+\sum_{\gamma \in \mathscr{F}_{I}} \tau_{\gamma} \int_{\gamma} \llbracket \mathrm{p}_{\mathscr{T}}-\mathrm{p}_{\mathscr{T}, m} \rrbracket_{\gamma}^{2} \\
=-\left(\mathcal{A}_{F}\left(\theta \mathscr{T} ; \mathbf{u}_{\mathscr{T}}, \mathbf{u}_{\mathscr{T}}-\mathbf{u}_{\mathscr{T}, m}\right)-\mathcal{A}_{F}\left(\theta_{\mathscr{T}, m} ; \mathbf{u}_{\mathscr{T}}, \mathbf{u}_{\mathscr{T}}-\mathbf{u}_{\mathscr{T}, m}\right)\right) \\
\quad-C_{F}\left(\mathscr{L}\left(\boldsymbol{\zeta}_{\mathscr{T}}-\zeta_{\mathscr{T}, m}, \chi \mathscr{T}-\chi \mathscr{T}, m\right) ; \mathbf{u}_{\mathscr{T}}, \mathbf{u}_{\mathscr{T}}-\mathbf{u}_{\mathscr{T}, m}\right)+\mathcal{G}_{F}\left(\theta_{\mathscr{T}}-\theta_{\mathscr{T}, m}, \mathbf{u}_{\mathscr{T}}-\mathbf{u}_{\mathscr{T}, m}\right) .
\end{aligned}
$$

We apply (32) in the left hand side of the above equality. The terms on the right hand side are bounded using the triangle inequality, (30), (26), and (24). We then divide by $\left\|\left(\mathbf{u}_{\mathscr{T}}-\mathbf{u}_{\mathscr{T}, m}, \mathrm{p}_{\mathscr{T}}-\mathrm{p}_{\mathscr{T}, m}\right)\right\|_{\mathcal{N}(\mathscr{T})}$ and get

$$
\begin{aligned}
& \left\|\left(\mathbf{u}_{\mathscr{T}}-\mathbf{u}_{\mathscr{T}, m}, \mathrm{p}_{\mathscr{T}}-\mathrm{p}_{\mathscr{T}, m}\right)\right\|_{\mathcal{N}(\mathscr{T})} \leq C_{\varepsilon} C_{\varepsilon, l i p}\left\|\theta_{\mathscr{T}}-\theta_{\mathscr{T}, m}\right\|_{\mathcal{T}(\mathscr{T})}\left\|\mathbf{u}_{\mathscr{T}}\right\|_{W^{1, \infty}(\Omega)} \\
& +\tilde{C}_{C}\left\|\left(\zeta_{\mathscr{T}}-\zeta_{\mathscr{T}, m}, \chi \mathscr{T}-\chi \mathscr{T}, m\right)\right\|_{\mathcal{N}(\mathscr{T})}\left\|\mathbf{u}_{\mathscr{T}}\right\|_{\boldsymbol{L}^{3}(\Omega)}+\tilde{C}_{g}\|\mathbf{g}\|_{L^{2}(\Omega)}\left\|\theta_{\mathscr{T}}-\theta_{\mathscr{T}, m}\right\|_{\mathcal{T}(\mathscr{T})}
\end{aligned}
$$

Since $\left\|\left(\boldsymbol{\zeta}_{\mathscr{T}}-\zeta_{\mathscr{T}, m}, \chi \mathscr{T}-\chi \chi_{\mathscr{T}, m}\right)\right\|_{\mathcal{N}(\mathscr{T})} \stackrel{m \rightarrow \infty}{\longrightarrow} 0$ and $\left\|\theta_{\mathscr{T}}-\theta_{\mathscr{T}, m}\right\|_{\mathcal{T}(\mathscr{T})} \stackrel{m \rightarrow \infty}{\longrightarrow} 0$, then $\left(\mathbf{u}_{\mathscr{T}, m}, \mathrm{p}_{\mathscr{T}, m}\right) \stackrel{m \rightarrow \infty}{\longrightarrow}\left(\mathbf{u}_{\mathscr{T}}, \mathrm{p}_{\mathscr{T}}\right)$.

To prove that $\mathrm{t}_{\mathscr{T}, m}$ converges to $\mathrm{t}_{\mathscr{T}}$, we subtract the equations appearing in (43), considering the third equation of (41), then we take as test function $\psi_{\mathscr{T}}=\mathrm{t}_{\mathscr{T}}-\mathrm{t}_{\mathscr{T}, m}$ to obtain

$$
\begin{aligned}
& \left.\mathcal{A}_{T}\left(\theta_{\mathscr{T}} ; \mathrm{t}_{\mathscr{T}}, \mathrm{t}_{\mathscr{T}}-\mathrm{t}_{\mathscr{T}, m}\right)-\mathcal{A}_{T}\left(\theta_{\mathscr{T}, m} ; \mathrm{t}_{\mathscr{T}, m}, \mathrm{t}_{\mathscr{T}}-\mathrm{t}_{\mathscr{T}, m}\right)+C_{T}\left(\mathscr{L}_{\left(\zeta_{\mathscr{T}}, \chi \mathscr{T}\right)}\right) \mathrm{t}_{\mathscr{T}}, \mathrm{t}_{\mathscr{T}}-\mathrm{t}_{\mathscr{T}, m}\right) \\
& -C_{T}\left(\mathscr{L}\left(\boldsymbol{\zeta}_{\mathscr{T}, m}, \chi \mathscr{T}_{, m}\right) ; \mathrm{t}_{\mathscr{T}, m}, \mathrm{t}_{\mathscr{T}}-\mathrm{t}_{\mathscr{T}, m}\right)+\mathcal{S}_{T}\left(\mathrm{t}_{\mathscr{T}}-\mathrm{t}_{\mathscr{T}, m}, \mathrm{t}_{\mathscr{T}}-\mathrm{t}_{\mathscr{T}, m}\right)=0
\end{aligned}
$$

We add and subtract the terms $\mathcal{A}_{T}\left(\theta_{\mathscr{T}, m} ; \mathrm{t}_{\mathscr{T}}, \mathrm{t}_{\mathscr{T}}-\mathrm{t}_{\mathscr{T}, m}\right)$ and $C_{T}\left(\mathscr{L}\left(\zeta_{\mathscr{T}, m}, \chi \mathscr{T}_{, m}\right) ; \mathrm{t}_{\mathscr{T}}, \mathrm{t}_{\mathscr{T}}-\mathrm{t}_{\mathscr{T}, m}\right)$, we consider the fact that $\mathscr{L}(\cdot)$ is linear and the definition of $\mathcal{A}_{T}$ given in (10) to obtain

$$
\begin{aligned}
& \mathcal{A}_{T}\left(\theta_{\mathscr{T}} ; \mathrm{t}_{\mathscr{T}}, \mathrm{t}_{\mathscr{T}}-\mathrm{t}_{\mathscr{T}, m}\right)-\mathcal{A}_{T}\left(\theta_{\mathscr{T}, m} ; \mathrm{t}_{\mathscr{T}}, \mathrm{t}_{\mathscr{T}}-\mathrm{t}_{\mathscr{T}, m}\right)+\mathcal{A}_{T}\left(\theta_{\mathscr{T}, m} ; \mathrm{t}_{\mathscr{T}}-\mathrm{t}_{\mathscr{T}, m}, \mathrm{t}_{\mathscr{T}}-\mathrm{t}_{\mathscr{T}, m}\right) \\
& \left.\left.+C_{T}\left(\mathscr{L}_{\left(\zeta_{\mathscr{T}}\right.}-\zeta_{\mathscr{T}, m}, \chi \mathscr{T}-\chi \mathscr{T}, m\right) ; \mathrm{t}_{\mathscr{T}}, \mathrm{t}_{\mathscr{T}}-\mathrm{t}_{\mathscr{T}, m}\right)+C_{T}\left(\mathscr{L}_{\left(\zeta_{\mathscr{T}, m}, \chi \mathscr{T}, m\right.}\right) ; \mathrm{t}_{\mathscr{T}}-\mathrm{t}_{\mathscr{T}, m}, \mathrm{t}_{\mathscr{T}}-\mathrm{t}_{\mathscr{T}, m}\right) \\
& +\mathcal{S}_{T}\left(\mathrm{t}_{\mathscr{T}}-\mathrm{t}_{\mathscr{T}, m}, \mathrm{t}_{\mathscr{T}}-\mathrm{t}_{\mathscr{T}, m}\right)=0 .
\end{aligned}
$$

Using (14) and reordering terms it follows that

$$
\begin{aligned}
\mathcal{A}_{T}\left(\theta_{\mathscr{T}, m} ; \mathrm{t}_{\mathscr{T}}-\mathrm{t}_{\mathscr{T}, m}, \mathrm{t}_{\mathscr{T}}-\mathrm{t}_{\mathscr{T}, m}\right)+\mathcal{S}_{T}\left(\mathrm{t}_{\mathscr{T}}-\mathrm{t}_{\mathscr{T}, m}, \mathrm{t}_{\mathscr{T}}-\mathrm{t}_{\mathscr{T}, m}\right) \\
=-\left(\mathcal{A}_{T}\left(\theta_{\mathscr{T}} ; \mathrm{t}_{\mathscr{T}}, \mathrm{t}_{\mathscr{T}}-\mathrm{t}_{\mathscr{T}, m}\right)-\mathcal{A}_{T}\left(\theta_{\mathscr{T}, m} ; \mathrm{t}_{\mathscr{T}}, \mathrm{t}_{\mathscr{T}}-\mathrm{t}_{\mathscr{T}, m}\right)\right) \\
\left.-C_{T}\left(\mathscr{L}_{\left(\zeta_{\mathscr{T}}\right.}-\zeta_{\mathscr{T}, m}, \chi \mathscr{T}-\chi \mathscr{T}, m\right) ; \mathrm{t}_{\mathscr{T}}, \mathrm{t}_{\mathscr{T}}-\mathrm{t}_{\mathscr{T}, m}\right) .
\end{aligned}
$$

Using (33) on the left-hand side and, (31) and (27) on the right-hand side, and simplifying the term $\left\|\mathrm{t}_{\mathscr{T}}-\mathrm{t}_{\mathscr{T}, m}\right\|_{\mathcal{T}(\mathscr{T})}$ we arrive at

$$
\begin{aligned}
\tilde{C}_{c o, T}\left\|\mathrm{t}_{\mathscr{T}}-\mathrm{t}_{\mathscr{T}, m}\right\|_{\mathcal{T}(\mathscr{T})} \leq C_{K} C_{\kappa, l i p}\left\|\theta_{\mathscr{T}}-\theta_{\mathscr{T}, m}\right\|_{\mathcal{T}(\mathscr{T})}\left\|\mathrm{t}_{\mathscr{T}}\right\|_{W^{1, \infty}(\Omega)} \\
+\tilde{C}_{\mathrm{c}}\left\|\left(\zeta_{\mathscr{T}}-\zeta_{\mathscr{T}, m}, \chi \mathscr{T}-\chi \mathscr{T}, m\right)\right\|_{\mathcal{N}(\mathscr{T})}\left\|\mathrm{t}_{\mathscr{T}}\right\|_{L^{3}(\Omega)} .
\end{aligned}
$$

Since $\left\|\left(\zeta_{\mathscr{T}}-\zeta_{\mathscr{T}, m}, \chi \mathscr{T}-\chi \mathscr{T}_{, m}\right)\right\|_{\mathcal{N}(\mathscr{T})} \stackrel{m \rightarrow \infty}{\longrightarrow} 0$ and $\left\|\theta_{\mathscr{T}}-\theta_{\mathscr{T}, m}\right\|_{\mathcal{T}(\mathscr{T})} \stackrel{m \rightarrow \infty}{\longrightarrow} 0$, then $\mathrm{t}_{\mathscr{T}, m} \stackrel{m \rightarrow \infty}{\longrightarrow} \mathrm{t}_{\mathscr{T}}$. This completes the proof. 


\section{A priori error estimation}

This section is devoted to proving the convergence of Method (15), and its rate of convergence. We start by remarking that, thanks to the hypotheses made on the stabilizing terms $\mathcal{S}_{F}$ and $\mathcal{S}_{T}$, they satisfy the following estimates:

$$
\begin{cases}\left|\mathcal{S}_{F}(\mathbf{u}, \boldsymbol{\xi})\right| \leq C h\|\mathbf{u}\|_{\boldsymbol{H}^{2}(\Omega)}\|(\boldsymbol{\xi}, \phi)\|_{\mathcal{N}(\mathscr{T})}, & \forall(\boldsymbol{\xi}, \phi) \in \boldsymbol{V}(\mathscr{T}) \times Q(\mathscr{T}), \\ \left|\mathcal{S}_{T}(\mathrm{t}, \psi)\right| \leq C h\|t\|_{H^{2}(\Omega)}\|\psi\|_{\mathcal{T}(\mathscr{T})}, & \forall \psi \in V(\mathscr{T}) \times Q(\mathscr{T}),\end{cases}
$$

whenever the exact solution $\mathbf{u}, t$ of (7) is regular enough.

Theorem 3. Let $(\mathbf{u}, \mathrm{p}, \mathrm{t}) \in\left[\boldsymbol{H}_{0}^{1}(\Omega) \cap \boldsymbol{H}^{2}(\Omega) \cap \boldsymbol{W}^{1, \infty}(\Omega)\right] \times\left[H^{1}(\Omega) \cap L_{0}^{2}(\Omega)\right] \times\left[H^{2}(\Omega) \cap W^{1, \infty}(\Omega)\right]$ be a solution of (9) and let $\left(\mathbf{u}_{\mathscr{T}}, \mathrm{p}_{\mathscr{T}}, \mathrm{t}_{\mathscr{T}}\right) \in \boldsymbol{V}_{0}(\mathscr{T}) \times Q(\mathscr{T}) \times V(\mathscr{T})$ be a solution of $(15)$. Let us suppose that

$$
\max \left\{\|\mathbf{g}\|_{L^{2}(\Omega)},\|\mathbf{u}\|_{W^{1, \infty}(\Omega)},\|t\|_{W^{1, \infty}(\Omega)}\right\} \leq L,
$$

where $L$ is small enough (a more especific expression for $L$ is given in (70)). Then, there exists a constant $C>0$ such that

$$
\left\|\left(\mathbf{u}-\mathbf{u}_{\mathscr{T}}, \mathrm{p}-\mathrm{p}_{\mathscr{T}}\right)\right\|_{\mathcal{N}(\mathscr{T})}+\left\|\mathrm{t}-\mathrm{t}_{\mathscr{T}}\right\|_{\mathcal{T}(\mathscr{T})} \leq C h\left(\left\|\mathbf{u}_{H^{2}(\Omega)}+\right\| \mathrm{p}\left\|_{H^{1}(\Omega)}+\right\| \mathrm{t} \|_{H^{2}(\Omega)}\right) .
$$

Proof. We denote $\boldsymbol{e}^{\mathbf{u}}=\mathbf{u}-\mathbf{u}_{\mathscr{T}}, e^{\mathrm{p}}=\mathrm{p}-\mathrm{p}_{\mathscr{T}}$ and $e^{\mathrm{t}}=\mathrm{t}-\mathrm{t}_{\mathscr{T}}$. These errors terms will be split as

$$
\left\{\begin{array}{lllll}
e^{\mathbf{u}}=\eta^{\mathbf{u}}+e_{\mathscr{T}}^{\mathbf{u}} & \text { with } & \eta^{\mathbf{u}}:=\mathbf{u}-i_{h}(\mathbf{u}) & \text { and } & \boldsymbol{e}_{\mathscr{T}}^{\mathbf{u}}:=i_{h}(\mathbf{u})-\mathbf{u}_{\mathscr{T}}, \\
e^{\mathrm{p}}=\eta^{\mathrm{p}}+e_{\mathscr{T}}^{\mathrm{p}} & \text { with } & \eta^{\mathrm{p}}:=\mathrm{p}-\Pi_{\mathscr{T}}(p) & \text { and } \quad e_{\mathscr{p}}^{\mathrm{p}}:=\Pi_{\mathscr{T}}(\mathrm{p})-\mathrm{p}_{\mathscr{T}}, \\
e^{\mathrm{t}}=\eta^{\mathrm{t}}+e_{\mathscr{T}}^{\mathrm{t}} & \text { with } & \eta^{\mathrm{t}}:=\mathrm{t}-i_{h}(\mathrm{t}) & \text { and } \quad e_{\mathscr{T}}^{\mathrm{t}}:=i_{h}(\mathrm{t})-\mathrm{t}_{\mathscr{T}},
\end{array}\right.
$$

for which it is easy to see that $\left(\boldsymbol{e}_{\mathscr{T}}^{\mathbf{u}}, e_{\mathscr{T}}^{\mathrm{p}}, e_{\mathscr{T}}^{\mathrm{t}}\right) \in \boldsymbol{V}_{0}(\mathscr{T}) \times Q(\mathscr{T}) \times V_{0}(\mathscr{T})$. Now, using (32), adding and subtracting the term $\mathcal{B}_{F}\left(\boldsymbol{e}_{\mathscr{T}}^{\mathbf{u}}, e_{\mathscr{T}}^{\mathrm{p}}\right)$, next using (12) and (47), we obtain

$$
\begin{aligned}
\left\|\left(\boldsymbol{e}_{\mathscr{T}}^{\mathbf{u}}, e_{\mathscr{T}}^{\mathrm{p}}\right)\right\|_{\mathcal{N}(\mathscr{T})}^{2} & \leq \mathcal{A}_{F}\left(\mathrm{t}_{\mathscr{T}} ; \boldsymbol{e}_{\mathscr{T}}^{\mathbf{u}}, \boldsymbol{e}_{\mathscr{T}}^{\mathbf{u}}\right)+\mathcal{S}_{F}\left(\boldsymbol{e}_{\mathscr{T}}^{\mathbf{u}}, \boldsymbol{e}_{\mathscr{T}}^{\mathbf{u}}\right)+\sum_{\gamma \in \mathcal{F}_{I}} \tau_{\gamma} \int_{\gamma} \llbracket e_{\mathscr{T}}^{\mathrm{p}} \mathbb{1}_{\gamma} \llbracket e_{\mathscr{T}}^{\mathrm{p}} \rrbracket_{\gamma} \\
& +\mathcal{C}_{F}\left(\mathscr{L}\left(\mathbf{u}_{\mathscr{T}}, \mathrm{p}_{\mathscr{T}}\right) ; \boldsymbol{e}_{\mathscr{T}}^{\mathbf{u}}, \boldsymbol{e}_{\mathscr{T}}^{\mathbf{u}}\right)-\mathcal{B}_{F}\left(\boldsymbol{e}_{\mathscr{T}}^{\mathbf{u}}, e_{\mathscr{T}}^{\mathrm{p}}\right)+\mathcal{B}_{F}\left(\boldsymbol{e}_{\mathscr{T}}^{\mathbf{u}}, e_{\mathscr{T}}^{\mathrm{p}}\right)=T_{1}-T_{2},
\end{aligned}
$$

where $T_{1}$ and $T_{2}$ are given by

$$
\begin{aligned}
T_{1}= & \mathcal{A}_{F}\left(\mathrm{t}_{\mathscr{T}} ; \boldsymbol{e}^{\mathbf{u}}, \boldsymbol{e}_{\mathscr{T}}^{\mathbf{u}}\right)+\mathcal{S}_{F}\left(\boldsymbol{e}^{\mathbf{u}}, \boldsymbol{e}_{\mathscr{T}}^{\mathbf{u}}\right)+\sum_{\gamma \in \mathcal{F}_{I}} \tau_{\gamma} \int_{\gamma} \llbracket e^{\mathrm{p}} \rrbracket_{\gamma} \llbracket e_{\mathscr{T}}^{\mathrm{p}} \rrbracket_{\gamma} \\
& +\mathcal{C}_{F}\left(\mathscr{L}\left(\mathbf{u}_{\mathscr{T}}, \mathrm{p}_{\mathscr{T}}\right) ; \boldsymbol{e}^{\mathbf{u}}, \boldsymbol{e}_{\mathscr{T}}^{\mathbf{u}}\right)-\mathcal{B}_{F}\left(\boldsymbol{e}_{\mathscr{T}}^{\mathbf{u}}, e^{\mathrm{p}}\right)+\mathcal{B}_{F}\left(\boldsymbol{e}^{\mathbf{u}}, e_{\mathscr{T}}^{\mathrm{p}}\right), \\
T_{2}= & \mathcal{A}_{F}\left(\mathrm{t}_{\mathscr{T}} ; \boldsymbol{\eta}^{\mathbf{u}}, \boldsymbol{e}_{\mathscr{T}}^{\mathbf{u}}\right)+\mathcal{S}_{F}\left(\boldsymbol{\eta}^{\mathbf{u}}, \boldsymbol{e}_{\mathscr{T}}^{\mathbf{u}}\right)+\sum_{\gamma \in \mathscr{F}_{I}} \tau_{\gamma} \int_{\gamma} \llbracket \eta^{\mathrm{p}} \rrbracket_{\gamma} \llbracket e_{\mathscr{T}}^{\mathrm{p}} \rrbracket_{\gamma} \\
& +\mathcal{C}_{F}\left(\mathscr{L}\left(\mathbf{u}_{\mathscr{T}}, \mathrm{p}_{\mathscr{T}}\right) ; \boldsymbol{\eta}^{\mathbf{u}}, \boldsymbol{e}_{\mathscr{T}}^{\mathbf{u}}\right)-\mathcal{B}_{F}\left(\boldsymbol{e}_{\mathscr{T}}^{\mathbf{u}}, \eta^{\mathrm{p}}\right)+\mathcal{B}_{F}\left(\boldsymbol{\eta}^{\mathbf{u}}, e_{\mathscr{T}}^{\mathrm{p}}\right)
\end{aligned}
$$

Hence,

$$
\left\|\left(\boldsymbol{e}_{\mathscr{T}}^{\mathbf{u}}, e_{\mathscr{T}}^{\mathrm{p}}\right)\right\|_{\mathcal{N}(\mathscr{T})}^{2} \leq\left|T_{1}\right|+\left|T_{2}\right| .
$$

We first rewrite the term $T_{1}$ as follows,

$$
\begin{aligned}
& T_{1}=\mathcal{A}_{F}\left(\mathrm{t}_{\mathscr{T}} ; \mathbf{u}, \boldsymbol{e}_{\mathscr{T}}^{\mathbf{u}}\right)+\mathcal{C}_{F}\left(\mathscr{L}\left(\mathbf{u}_{\mathscr{T}}, \mathrm{p} \mathscr{T}\right) ; \mathbf{u}, \boldsymbol{e}_{\mathscr{T}}^{\mathbf{u}}\right)+\mathcal{S}_{F}\left(\mathbf{u}, \boldsymbol{e}_{\mathscr{T}}^{\mathbf{u}}\right)+\sum_{\gamma \in \mathcal{F}_{I}} \tau_{\gamma} \int_{\gamma} \llbracket \mathrm{p} \rrbracket_{\gamma} \llbracket e_{\mathscr{T}}^{\mathrm{p}} \rrbracket_{\gamma} \\
& -\mathcal{B}_{F}\left(\boldsymbol{e}_{\mathscr{T}}^{\mathbf{u}}, \mathrm{p}\right)+\mathcal{B}_{F}\left(\mathbf{u}, e_{\mathscr{T}}^{\mathrm{p}}\right) \\
& -\left[\mathcal{A}_{F}\left(\mathrm{t}_{\mathscr{T}} ; \mathbf{u}_{\mathscr{T}}, \boldsymbol{e}_{\mathscr{T}}^{\mathbf{u}}\right)+\mathcal{C}_{F}\left(\mathscr{L}\left(\mathbf{u}_{\mathscr{T}}, \mathrm{p}_{\mathscr{T}}\right) ; \mathbf{u}_{\mathscr{T}}, \boldsymbol{e}_{\mathscr{T}}^{\mathbf{u}}\right)+\mathcal{S}_{F}\left(\mathbf{u}_{\mathscr{T}}, \boldsymbol{e}_{\mathscr{T}}^{\mathbf{u}}\right)+\sum_{\gamma \in \mathcal{F}_{I}} \tau_{\gamma} \int_{\gamma} \llbracket p_{\mathscr{T}} \rrbracket_{\gamma} \llbracket e_{\mathscr{T}}^{\mathrm{p}} \rrbracket_{\gamma}\right. \\
& \left.-\mathcal{B}_{F}\left(\boldsymbol{e}_{\mathscr{T}}^{\mathbf{u}}, \mathrm{p}_{\mathscr{T}}\right)+\mathcal{B}_{F}\left(\mathbf{u}_{\mathscr{T}}, e_{\mathscr{T}}^{\mathrm{p}}\right)\right] .
\end{aligned}
$$


Note that $\mathcal{B}_{F}\left(\mathbf{u}, e_{\mathscr{T}}^{\mathrm{p}}\right)=0, \mathcal{A}_{F}\left(\mathrm{t}_{\mathscr{T}} ; \mathbf{u}_{\mathscr{T}}, \boldsymbol{e}_{\mathscr{T}}^{\mathbf{u}}\right)+\mathcal{C}_{F}\left(\mathscr{L}\left(\mathbf{u}_{\mathscr{T}}, \mathrm{p}_{\mathscr{T}}\right) ; \mathbf{u}_{\mathscr{T}}, \boldsymbol{e}_{\mathscr{T}}^{\mathbf{u}}\right)+\mathcal{S}_{F}\left(\mathbf{u}_{\mathscr{T}}, \boldsymbol{e}_{\mathscr{T}}^{\mathbf{u}}\right)-\mathcal{B}_{F}\left(\boldsymbol{e}_{\mathscr{T}}^{\mathbf{u}}, \mathrm{p}_{\mathscr{T}}\right)=\mathcal{G}_{F}\left(\mathrm{t}_{\mathscr{T}}, \boldsymbol{e}_{\mathscr{T}}^{\mathbf{u}}\right)$ and $\mathcal{B}_{F}\left(\mathbf{u}_{\mathscr{T}}, e_{\mathscr{T}}^{\mathrm{p}}\right)+\sum_{\gamma \in \mathcal{F}_{I}} \tau_{\gamma} \int_{\gamma} \llbracket \mathrm{p}_{\mathscr{T}} \mathbb{1}_{\gamma} \llbracket e_{\mathscr{T}}^{\mathrm{p}} \rrbracket_{\gamma}=0$, since $\left(\mathbf{u}_{\mathscr{T}}, \mathrm{p}_{\mathscr{T}}, \mathrm{t}_{\mathscr{T}}\right)$ solves $(15)$. Considering this, we add and subtract the terms $\mathcal{A}_{F}\left(\mathrm{t} ; \mathbf{u}, \boldsymbol{e}_{\mathscr{T}}^{\mathbf{u}}\right)$ and $\mathcal{C}_{F}\left(\mathscr{L}(\mathbf{u}, \mathrm{p}) ; \mathbf{u}, \boldsymbol{e}_{\mathscr{T}}^{\mathbf{u}}\right)$ and reorder terms to obtain

$$
\begin{aligned}
T_{1} & =\mathcal{A}_{F}\left(\mathrm{t} ; \mathbf{u}, \boldsymbol{e}_{\mathscr{T}}^{\mathbf{u}}\right)+\mathcal{C}_{F}\left(\mathscr{L}(\mathbf{u}, \mathrm{p}) ; \mathbf{u}, \boldsymbol{e}_{\mathscr{T}}^{\mathbf{u}}\right)+\mathcal{S}_{F}\left(\mathbf{u}, \boldsymbol{e}_{\mathscr{T}}^{\mathbf{u}}\right)-\mathcal{B}_{F}\left(\boldsymbol{e}_{\mathscr{T}}^{\mathbf{u}}, \mathrm{p}\right)-\mathcal{G}_{F}\left(\mathrm{t}_{\mathscr{T}}, \boldsymbol{e}_{\mathscr{T}}^{\mathbf{u}}\right)+\sum_{\gamma \in \mathcal{F}_{I}} \tau_{\gamma} \int_{\gamma} \llbracket \mathrm{p} \rrbracket_{\gamma} \llbracket\left[e_{\mathscr{T}}^{\mathrm{p}} \mathbb{\rrbracket}_{\gamma}\right. \\
& -\left[\mathcal{A}_{F}\left(\mathrm{t} ; \mathbf{u}, \boldsymbol{e}_{\mathscr{T}}^{\mathbf{u}}\right)-\mathcal{A}_{F}\left(\mathrm{t}_{\mathscr{T}} ; \mathbf{u}, \boldsymbol{e}_{\mathscr{T}}^{\mathbf{u}}\right)+\mathcal{C}_{F}\left(\mathscr{L}\left(\mathbf{u}-\mathbf{u}_{\mathscr{T}}, \mathrm{p}-\mathrm{p}_{\mathscr{T}}\right) ; \mathbf{u}, \boldsymbol{e}_{\mathscr{T}}^{\mathbf{u}}\right)\right] .
\end{aligned}
$$

Now, since $\mathrm{p} \in H^{1}(\Omega)$ we have that $\llbracket p \rrbracket=0$ a.e. in $\gamma$, for all $\gamma \in \mathcal{F}_{I}$. Moreover, since $(\mathbf{u}, \mathrm{p}, \mathrm{t})$ solves $(9)$ it follows that

$$
T_{1}=\mathcal{G}_{F}\left(\mathrm{t}-\mathrm{t}_{\mathscr{T}}, \boldsymbol{e}_{\mathscr{T}}^{\mathbf{u}}\right)-\left(\mathcal{A}_{F}\left(\mathrm{t} ; \mathbf{u}, \boldsymbol{e}_{\mathscr{T}}^{\mathbf{u}}\right)-\mathcal{A}_{F}\left(\mathrm{t}_{\mathscr{T}} ; \mathbf{u}, \boldsymbol{e}_{\mathscr{T}}^{\mathbf{u}}\right)\right)-C_{F}\left(\mathscr{L}\left(\mathbf{u}-\mathbf{u}_{\mathscr{T}}, \mathrm{p}-\mathrm{p}_{\mathscr{T}}\right) ; \mathbf{u}, \boldsymbol{e}_{\mathscr{T}}^{\mathbf{u}}\right)+\mathcal{S}_{F}\left(\mathbf{u}, \boldsymbol{e}_{\mathscr{T}}^{\mathbf{u}}\right) .
$$

Applying (24), (30), (28), and (44) to (50) we obtain

$$
\begin{aligned}
\left|T_{1}\right| & \leq\left(\tilde{C}_{g}\|\mathbf{g}\|_{L^{2}(\Omega)}\left\|e^{\mathrm{t}}\right\|_{\mathcal{T}(\mathscr{T})}+C_{\varepsilon, l i p} C_{\varepsilon}\|\mathbf{u}\|_{W^{1, \infty}(\Omega)}\left\|e^{\mathrm{t}}\right\|_{\mathcal{T}(\mathscr{T})}\right. \\
& \left.+\tilde{C}_{C 2}\|\mathbf{u}\|_{W^{1, \infty}(\Omega)}\left\|\left(\boldsymbol{e}^{\mathbf{u}}, e^{\mathrm{p}}\right)\right\|_{\mathcal{N}(\mathscr{T})}+C h\|\|_{H^{2}(\Omega)}\right)\left\|\left(\boldsymbol{e}_{\mathscr{T}}^{\mathbf{u}}, e_{\mathscr{T}}^{\mathrm{p}}\right)\right\|_{\mathcal{N}(\mathscr{T})} .
\end{aligned}
$$

We next bound every term appearing in $T_{2}$. Using (8), the Cauchy-Schwarz inequality and the definition of the $\boldsymbol{H}^{1}(\Omega)$ norm, the fact that $\left\|\boldsymbol{\eta}^{\mathbf{u}}\right\|_{H^{1}(\Omega)} \leq C h\|\mathbf{u}\|_{H^{2}(\Omega)}$ (as a consecuence of (3)) and the definition of $\|(\cdot, \cdot)\|_{\mathcal{N}(\mathscr{T})}$ we get

$$
\left|\mathcal{A}_{F}\left(\mathrm{t}_{\mathscr{T}} ; \boldsymbol{\eta}^{\mathbf{u}}, \boldsymbol{e}_{\mathscr{T}}^{\mathbf{u}}\right)\right| \leq \varepsilon_{1}^{-1 / 2} \varepsilon_{2} \tilde{C}_{1} h\|\mathbf{u}\|_{\boldsymbol{H}^{2}(\Omega)}\left\|\left(\boldsymbol{e}_{\mathscr{T}}^{\mathbf{u}}, e_{\mathscr{T}}^{\mathrm{p}}\right)\right\|_{\mathcal{N}(\mathscr{T})} .
$$

Now, using the Cauchy-Schwarz inequality, the definition of $\|(\cdot, \cdot)\|_{\mathcal{N}(\mathscr{T})},(20),(18),(6)$ and (3), it follows that

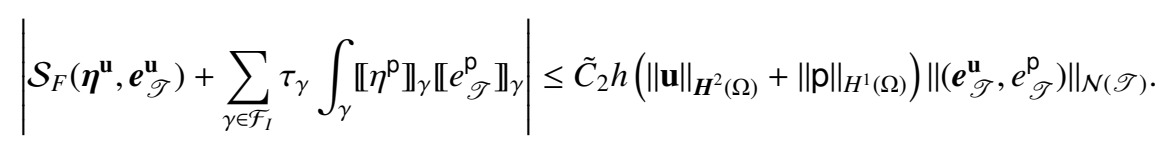

Next,

$$
\mathcal{B}_{F}\left(\boldsymbol{e}_{\mathscr{T}}^{\mathbf{u}}, \eta^{\mathrm{p}}\right)=0,
$$

since $\Pi_{\mathscr{T}}$ is the orthogonal $L^{2}$-projection onto the space of piecewise constant functions and $\operatorname{div} \boldsymbol{e}_{\mathscr{T}}^{\mathbf{u}} \in Q(\mathscr{T})$. To bound the term $\mathcal{B}_{F}\left(\boldsymbol{\eta}^{\mathbf{u}}, e_{\mathscr{T}}^{\mathrm{p}}\right)$, we integrate by parts, consider that $\left.e_{\mathscr{T}}^{\mathrm{p}}\right|_{K} \in \mathbb{P}_{0}(K)$ for all $K \in \mathscr{T}$ and that $\left.\boldsymbol{\eta}^{\mathbf{u}}\right|_{\Gamma}=0$, multiply and divide by the term $\tau_{\gamma}^{1 / 2}$, use the Cauchy-Scwharz inequality, and get to

$$
\left|\mathcal{B}_{F}\left(\boldsymbol{\eta}^{\mathbf{u}}, e_{\mathscr{T}}^{\mathrm{p}}\right)\right|=\left|\sum_{\gamma \in \mathcal{F}_{I}} \int_{\gamma} \llbracket e_{\mathscr{T}}^{\mathrm{p}} \mathbb{I}_{\gamma} \boldsymbol{\eta}^{\mathbf{u}} \cdot \boldsymbol{n}_{\gamma}\right| \leq\left\|\left(\boldsymbol{e}_{\mathscr{T}}^{\mathbf{u}}, e_{\mathscr{T}}^{\mathrm{p}}\right)\right\|_{\mathcal{N}(\mathscr{T})}\left(\sum_{\gamma \in \mathcal{F}_{I}} \frac{1}{\tau_{\gamma}}\left\|\boldsymbol{\eta}^{\mathbf{u}}\right\|_{\boldsymbol{L}^{2}(\gamma)}^{2}\right)^{1 / 2} .
$$

Now, using (5), (3) and $\tau_{\gamma}=h_{\gamma} / 12$, we obtain $\frac{1}{\tau_{\gamma}}\left\|\boldsymbol{\eta}^{\mathbf{u}}\right\|_{\mathbf{L}^{2}(\gamma)}^{2} \leq C h_{K}^{2}\|\mathbf{u}\|_{\boldsymbol{H}^{2}(K)}^{2}$, for all $\gamma \in \mathcal{F}_{I} \cap \mathcal{F}_{K}$. Therefore,

$$
\left|\mathcal{B}_{F}\left(\boldsymbol{\eta}^{\mathbf{u}}, e_{\mathscr{T}}^{\mathrm{p}}\right)\right| \leq \tilde{C}_{3} h\left\|\left(\boldsymbol{e}_{\mathscr{T}}^{\mathbf{u}}, e_{\mathscr{T}}^{\mathrm{p}}\right)\right\|_{\mathcal{N}(\mathscr{T})}\|\mathbf{u}\|_{\boldsymbol{H}^{2}(\Omega)} .
$$

To bound the term $C_{F}\left(\mathscr{L}\left(\mathbf{u}_{\mathscr{T}}, \mathrm{p}_{\mathscr{T}}\right) ; \boldsymbol{\eta}^{\mathbf{u}}, \boldsymbol{e}_{\mathscr{T}}^{\mathbf{u}}\right)$ we use Hölder's inequality, (23) with $q=4$, (35), the Sobolev embedding and the Poincaré inequalities given in (4), and the fact that $\left(\mathbf{u}_{\mathscr{T}}, \mathrm{p}_{\mathscr{T}}\right)$ satisfy (35), we get

$$
\left|C_{F}\left(\mathscr{L}\left(\mathbf{u}_{\mathscr{T}}, \mathbf{p} \mathscr{T}_{\mathscr{T}}\right) ; \boldsymbol{\eta}^{\mathbf{u}}, \boldsymbol{e}_{\mathscr{T}}^{\mathbf{u}}\right)\right| \leq C_{F} \bar{C}_{4} C_{e m b} \tilde{C}_{P}\left\|\mathbf{t}_{\mathscr{T}, 1}\right\|_{\mathcal{T}(\mathscr{T})}\left\|\boldsymbol{\eta}^{\mathbf{u}}\right\|_{H^{1}(\Omega)}\left\|\nabla \boldsymbol{e}_{\mathscr{T}}^{\mathbf{u}}\right\|_{\tilde{L}^{2}(\Omega)},
$$

where $\bar{C}_{4}$ is given in (23). Then, using the definition of the $\boldsymbol{H}^{1}(\Omega)$ norm and (3), we obtain

$$
\left|C_{F}\left(\mathscr{L}\left(\mathbf{u}_{\mathscr{T}}, \mathrm{p}_{\mathscr{T}}\right) ; \boldsymbol{\eta}^{\mathbf{u}}, \boldsymbol{e}_{\mathscr{T}}^{\mathbf{u}}\right)\right| \leq \tilde{C}_{4} h \varepsilon_{1}^{-1 / 2}\left\|\mathrm{t}_{\mathscr{T}, 1}\right\|_{\mathcal{T}(\mathscr{T})}\|\mathbf{u}\|_{\boldsymbol{H}^{2}(\Omega)}\left\|\left(\boldsymbol{e}_{\mathscr{T}}^{\mathbf{u}}, e_{\mathscr{T}}^{\mathrm{p}}\right)\right\|_{\mathcal{N}(\mathscr{T})}
$$

Thus, replacing (52)-(56) in (48) it follows that

$$
\left|T_{2}\right| \leq C h\left(\|\mathbf{u}\|_{\boldsymbol{H}^{2}(\Omega)}+\|\mathrm{p}\|_{H^{1}(\Omega)}\right)\left\|\left(\boldsymbol{e}_{\mathscr{T}}^{\mathbf{u}}, e_{\mathscr{T}}^{\mathrm{p}}\right)\right\|_{\mathcal{N}(\mathscr{T})} .
$$


Hence, replacing (51) and (57) in (49) and simplifying the term $\left\|\left(\boldsymbol{e}_{\mathscr{T}}^{\mathbf{u}}, e_{\mathscr{T}}^{\mathrm{p}}\right)\right\|_{\mathcal{N}(\mathscr{T})}$ we arrive at

$$
\begin{aligned}
\left\|\left(\boldsymbol{e}_{\mathscr{T}}^{\mathbf{u}}, e_{\mathscr{T}}^{\mathrm{p}}\right)\right\|_{\mathcal{N}(\mathscr{T})} & \leq C h\left(\|\mathbf{u}\|_{H^{2}(\Omega)}+\|\mathrm{p}\|_{H^{1}(\Omega)}\right)+\left(C_{1}\|\mathbf{g}\|_{L^{2}(\Omega)}+C_{2}\|\mathbf{u}\|_{W^{1, \infty}(\Omega)}\right)\left\|e^{\mathrm{t}}\right\|_{\mathcal{T}(\mathscr{T})} \\
& +C_{3}\|\mathbf{u}\|_{W^{1, \infty}(\Omega)}\left\|\left(\boldsymbol{e}^{\mathbf{u}}, e^{\mathrm{p}}\right)\right\|_{\mathcal{N}(\mathscr{T})},
\end{aligned}
$$

where

$$
C_{1}=\tilde{C}_{g}, C_{2}=C_{\varepsilon, l i p} C_{\varepsilon} \text {, and } C_{3}=\tilde{C}_{C 2} .
$$

On the other hand, using (33), (14), and (47), we get

$$
\begin{aligned}
\tilde{C}_{c o, T}\left\|e_{\mathscr{T}}^{\mathrm{t}}\right\|_{\mathcal{T}(\mathscr{T})}^{2} & \leq \mathcal{A}_{T}\left(\mathrm{t} \mathscr{T} ; e_{\mathscr{T}}^{\mathrm{t}}, e_{\mathscr{T}}^{\mathrm{t}}\right)+\mathcal{S}_{T}\left(e_{\mathscr{T}}^{\mathrm{t}}, e_{\mathscr{T}}^{\mathrm{t}}\right)+C_{T}\left(\mathscr{L}\left(\mathbf{u}_{\mathscr{T}}, \mathrm{p}_{\mathscr{T}}\right) ; e_{\mathscr{T}}^{\mathrm{t}}, e_{\mathscr{T}}^{\mathrm{t}}\right) \\
& =T_{3}-T_{4},
\end{aligned}
$$

where $T_{3}$ and $T_{4}$ are given by

$$
T_{3}=\mathcal{A}_{T}\left(\mathrm{t} \mathscr{T} ; e^{\mathrm{t}}, e_{\mathscr{T}}^{\mathrm{t}}\right)+\mathcal{S}_{T}\left(e^{\mathrm{t}}, e_{\mathscr{T}}^{\mathrm{t}}\right)+C_{T}\left(\mathscr{L}\left(\mathbf{u}_{\mathscr{T}}, \mathrm{p}_{\mathscr{T}}\right) ; e^{\mathrm{t}}, e_{\mathscr{T}}^{\mathrm{t}}\right)
$$

and

$$
T_{4}=\mathcal{A}_{T}\left(\mathrm{t} \mathscr{T} ; \eta^{\dagger}, e_{\mathscr{T}}^{\mathrm{t}}\right)+\mathcal{S}_{T}\left(\eta^{\mathrm{t}}, e_{\mathscr{T}}^{\mathrm{t}}\right)+C_{T}\left(\mathscr{L}\left(\mathbf{u}_{\mathscr{T}}, \mathrm{p}_{\mathscr{T}}\right) ; \eta^{\mathrm{t}}, e_{\mathscr{T}}^{\mathrm{t}}\right)
$$

Hence,

$$
\left\|e_{\mathscr{T}}^{\mathrm{t}}\right\|_{\mathcal{T}(\mathscr{T})}^{2} \leq \tilde{C}_{c o, T}^{-1}\left(\left|T_{3}\right|+\left|T_{4}\right|\right) \text {. }
$$

First we bound the term $T_{3}$. To do so, we consider that $\left(\mathbf{u}_{\mathscr{T}}, \mathrm{p}_{\mathscr{T}}, \mathrm{t}_{\mathscr{T}}\right)$ solves $(15)$, add and substract the terms $\mathcal{A}_{T}\left(\mathrm{t} ; \mathrm{t}, e_{\mathscr{T}}^{\mathrm{t}}\right)$ and $C_{T}\left(\mathscr{L}(\mathbf{u}, \mathrm{p}) ; \mathrm{t}, e_{\mathscr{T}}^{\mathrm{t}}\right)$, and finally we consider that $(\mathbf{u}, \mathrm{p}, \mathrm{t})$ solves $(9)$ to obtain

$$
\begin{aligned}
T_{3}= & \mathcal{A}_{T}\left(\mathrm{t} \mathscr{T} ; \mathrm{t}, e_{\mathscr{T}}^{\mathrm{t}}\right)+\mathcal{S}_{T}\left(\mathrm{t}, e_{\mathscr{T}}^{\mathrm{t}}\right)+C_{T}\left(\mathscr{L}\left(\mathbf{u}_{\mathscr{T}}, \mathrm{p} \mathscr{T}\right) ; \mathrm{t}, e_{\mathscr{T}}^{\mathrm{t}}\right) \\
= & \mathcal{A}_{T}\left(\mathrm{t} ; \mathrm{t}, e_{\mathscr{T}}^{\mathrm{t}}\right)+C_{T}\left(\mathscr{L}(\mathbf{u}, \mathrm{p}) ; \mathrm{t}, e_{\mathscr{T}}^{\mathrm{t}}\right)+\mathcal{S}_{T}\left(\mathrm{t}, e_{\mathscr{T}}^{\mathrm{t}}\right)-\mathcal{A}_{T}\left(\mathrm{t} ; \mathrm{t}, e_{\mathscr{T}}^{\mathrm{t}}\right)+\mathcal{A}_{T}\left(\mathrm{t} \mathscr{T}_{\mathscr{T}} ; \mathrm{t}, e_{\mathscr{T}}^{\mathrm{t}}\right) \\
& \quad-C_{T}\left(\mathscr{L}\left(\mathbf{u}-\mathbf{u}_{\mathscr{T}}, \mathrm{p}-\mathrm{p} \mathscr{T}\right) ; \mathrm{t}, e_{\mathscr{T}}^{\mathrm{t}}\right) \\
= & -\mathcal{A}_{T}\left(\mathrm{t} ; \mathrm{t}, e_{\mathscr{T}}^{\mathrm{t}}\right)+\mathcal{A}_{T}\left(\mathrm{t}{ }_{\mathscr{T}} ; \mathrm{t}, e_{\mathscr{T}}^{\mathrm{t}}\right)-C_{T}\left(\mathscr{L}\left(\mathbf{u}-\mathbf{u}_{\mathscr{T}}, \mathrm{p}-\mathrm{p}{ }_{\mathscr{T}}\right) ; \mathrm{t}, e_{\mathscr{T}}^{\mathrm{t}}\right)+\mathcal{S}_{T}\left(\mathrm{t}, e_{\mathscr{T}}^{\mathrm{t}}\right) .
\end{aligned}
$$

From (31), (29) and (44) it follows that

$$
\left|T_{3}\right| \leq\left(C_{\kappa, l i p} C_{\kappa}\left\|e^{\mathrm{t}}\right\|_{\mathcal{T}(\mathscr{T})}\|\mathrm{t}\|_{W^{1, \infty}(\Omega)}+\tilde{C}_{\mathrm{c} 2}\|t\|_{W^{1, \infty}(\Omega)}\left\|\left(\boldsymbol{e}^{\mathbf{u}}, e^{\mathrm{p}}\right)\right\|_{\mathcal{N}(\mathscr{T})}+C h\|t\|_{H^{2}(\Omega)}\right)\left\|e_{\mathscr{T}}^{\mathrm{t}}\right\|_{\mathcal{T}(\mathscr{T})} .
$$

Now, we will bound every term appearing in $T_{4}$. From (8), the Cauchy-Schwarz inequality (3) and the definition of $\|\cdot\|_{\mathcal{T}(\mathscr{T})}$ it follows that

$$
\left|\mathcal{A}_{T}\left(\mathrm{t} \mathscr{T}_{F} ; \eta^{\mathrm{t}}, e_{\mathscr{T}}^{\mathrm{t}}\right)\right| \leq \overline{\bar{C}}_{1} \kappa_{2} \kappa_{1}^{-1 / 2} h\|\mathrm{t}\|_{H^{2}(\Omega)}\left\|e_{\mathscr{T}}^{\mathrm{t}}\right\|_{\mathcal{T}(\mathscr{T})} .
$$

From (20), (18) and (3) and the definition of the $\|\cdot\|_{\mathcal{T}(\mathscr{T})}$ norm, it follows that

$$
\left|\mathcal{S}_{T}\left(\eta^{\mathrm{t}}, e_{\mathscr{T}}^{\mathrm{t}}\right)\right| \leq \overline{\bar{C}}_{2} h\|t\|_{H^{2}(\Omega)}\left\|e_{\mathscr{T}}^{\mathrm{t}}\right\|_{\mathcal{T}(\mathscr{T})} .
$$

To bound the term $C_{T}\left(\mathscr{L}\left(\mathbf{u}_{\mathscr{T}}, \mathrm{p}_{\mathscr{T}}\right) ; \eta^{\mathrm{t}}, e_{\mathscr{T}}^{\mathrm{t}}\right)$ we follow the same steps used to get (56), and we obtain

$$
\left|C_{T}\left(\mathscr{L}\left(\mathbf{u}_{\mathscr{T}}, \mathrm{p}, \mathscr{T}\right) ; \eta^{\dagger}, e_{\mathscr{T}}^{\mathrm{t}}\right)\right| \leq \overline{\bar{C}}_{3} h \kappa_{1}^{-1 / 2}\left\|\mathrm{t}_{\mathscr{T}, 1}\right\|_{\mathcal{T}(\mathscr{T})}\|\mathrm{t}\|_{H^{2}(\Omega)}\left\|e_{\mathscr{T}}^{\mathrm{t}}\right\|_{\mathcal{T}(\mathscr{T})} .
$$

Thus, replacing (63)-(65) in (60) we get

$$
\left|T_{4}\right| \leq C h|| t\left\|_{H^{2}(\Omega)}\right\| e_{\mathscr{T}}^{\mathrm{t}} \|_{\mathcal{T}(\mathscr{T})} .
$$

Hence, replacing (62) and (66) in (61), and simplifying the term $\left\|e_{\mathscr{T}}^{\mathrm{t}}\right\|_{\mathcal{T}(\mathscr{T})}$, it follows that

$$
\left\|e_{\mathscr{T}}^{\mathrm{t}}\right\|_{\mathcal{T}(\mathscr{T})} \leq C h\|t\|_{H^{2}(\Omega)}+C_{4}\left\|e^{\mathrm{t}}\right\|_{\mathcal{T}(\mathscr{T})}\|t\|_{W^{1, \infty}(\Omega)}+C_{5}\|t\|_{W^{1, \infty}(\Omega)}\left\|\left(e^{\mathbf{u}}, e^{\mathrm{p}}\right)\right\|_{\mathcal{N}(\mathscr{T})},
$$


where

$$
C_{4}=\tilde{C}_{c o, T}^{-1} C_{\kappa, l i p} C_{K} \quad \text { and } \quad C_{5}=\tilde{C}_{c o, T}^{-1} \tilde{C}_{\mathrm{c} 2} .
$$

We have bounded the $\boldsymbol{e}_{\mathscr{T}}^{\mathbf{u}}, e_{\mathscr{T}}^{\mathrm{p}}$ and $e_{\mathscr{T}}^{\mathrm{t}}$ components of the total error. In addition, using (3),(4) and (44), we get

$$
\left\|\left(\boldsymbol{\eta}^{\mathbf{u}}, \eta^{\mathrm{p}}\right)\right\|_{\mathcal{N}(\mathscr{T})} \leq C h\left(\|\mathbf{u}\|_{\boldsymbol{H}^{2}(\Omega)}+\|\mathrm{p}\|_{H^{1}(\Omega)}\right) \text { and }\left\|\eta^{\mathrm{t}}\right\|_{\mathcal{T}(\mathscr{T})} \leq C h\|t\|_{H^{2}(\Omega)} .
$$

Therefore, from the triangle inequality, (58), (67) and (69), we obtain

$$
\begin{aligned}
\left\|\left(\boldsymbol{e}^{\mathbf{u}}, e^{\mathrm{p}}\right)\right\|_{\mathcal{N}(\mathscr{T})}+\left\|e^{\mathrm{t}}\right\|_{\mathcal{T}(\mathscr{T})} \leq C h\left(\|\mathbf{u}\|_{H^{2}(\Omega)}+\|\mathrm{p}\|_{H^{1}(\Omega)}+\|t\|_{H^{2}(\Omega)}\right) \\
+\left(C_{1}\|\mathbf{g}\|_{L^{2}(\Omega)}+C_{2}\|\mathbf{u}\|_{W^{1, \infty}(\Omega)}\right)\left\|e^{\mathrm{t}}\right\|_{\mathcal{T}(\mathscr{T})} \\
+C_{3}\|\mathbf{u}\|_{W^{1, \infty}(\Omega)}\left\|\left(e^{\mathbf{u}}, e^{\mathfrak{p}}\right)\right\|_{\mathcal{N}(\mathscr{T})} \\
+C_{4}\|t\|\left\|_{W^{1, \infty}(\Omega)}\right\| e^{\mathrm{t}}\left\|_{\mathcal{T}(\mathscr{T})}+C_{5}\right\| t\left\|_{W^{1, \infty}(\Omega)}\right\|\left(\boldsymbol{e}^{\mathbf{u}}, e^{\mathfrak{p}}\right) \|_{\mathcal{N}(\mathscr{T})} .
\end{aligned}
$$

Finally, from (45) it follows that

$$
\begin{aligned}
\left\|\left(\boldsymbol{e}^{\mathbf{u}}, e^{\mathrm{p}}\right)\right\|_{\mathcal{N}(\mathscr{T})}+\left\|e^{\mathrm{t}}\right\|_{\mathcal{T}(\mathscr{T})} \leq C h\left(\|\mathbf{u}\|_{H^{2}(\Omega)}+\|\mathrm{p}\|_{H^{1}(\Omega)}+\|\mathrm{t}\|_{H^{2}(\Omega)}\right) \\
+L\left(C_{1}+C_{2}+C_{4}\right)\left\|e^{\mathrm{t}}\right\|_{\mathcal{T}(\mathscr{T})}+L\left(C_{3}+C_{5}\right)\left\|\left(\boldsymbol{e}^{\mathbf{u}}, e^{\mathrm{p}}\right)\right\|_{\mathcal{N}(\mathscr{T}) .}
\end{aligned}
$$

Now, if

$$
L<\min \left\{\frac{1}{C_{1}+C_{2}+C_{4}}, \frac{1}{C_{3}+C_{5}}\right\},
$$

where $C_{1}, C_{2}, C_{3}, C_{4}$ and $C_{5}$ are given in (59) and (68), then (46) follows.

This last result proved the optimal convergence of the discrete solution $\left(\mathbf{u}_{\mathscr{T}}, \mathrm{p}_{\mathscr{T}}, \mathrm{t}_{\mathscr{T}}\right)$ provided by (15) to the exact solution $(\mathbf{u}, \mathrm{p}, \mathrm{t})$ of (7). Now, as was mentioned earlier, the method also provides a divergence-free velocity field, given by $\mathscr{L}\left(\mathbf{u}_{\mathscr{T}}, \mathrm{p}_{\mathscr{T}}\right)$, which also converges optimally to $\mathbf{u}$. This is proven in the next result.

Theorem 4. There exist a positive constant $C$, such that

$$
\left(\sum_{K \in \mathscr{T}}\left\|\nabla\left(\mathbf{u}-\mathscr{L}\left(\mathbf{u}_{\mathscr{T}}, \mathrm{p}_{\mathscr{T}}\right)\right)\right\|_{L^{L^{2}(K)}}^{2}\right)^{1 / 2} \leq C\left(\left\|\nabla\left(\mathbf{u}-\mathbf{u}_{\mathscr{T}}\right)\right\|_{L^{2}(K)}^{2}+\sum_{\gamma \in \mathcal{F}_{I}} \tau_{\gamma}\left\|\llbracket \mathrm{p}-\mathrm{p}_{\mathscr{T}}\right\|_{\gamma} \|_{L^{2}(\gamma)}^{2}\right)^{1 / 2} .
$$

Proof. The result follows directly from considering that since $\mathrm{p} \in H^{1}(\Omega)$, then $\llbracket \mathrm{p} \rrbracket_{\gamma}=0$ for all $\gamma \in \mathcal{F}_{I}$, which implies that $\mathbf{u}=\mathscr{L}(\mathbf{u}, \mathrm{p})$. Then, since $\mathscr{L}(\cdot)$ is linear, and using (22), we get

$$
\begin{aligned}
& \sum_{K \in \mathscr{T}}\left\|\nabla\left(\mathbf{u}-\mathscr{L}\left(\mathbf{u}_{\mathscr{T}}, \mathrm{p}_{\mathscr{T}}\right)\right)\right\|_{\tilde{L}^{2}(K)}^{2}=\sum_{K \in \mathscr{T}}\left\|\nabla\left(\mathscr{L}(\mathbf{u}, \mathrm{p})-\mathscr{L}\left(\mathbf{u}_{\mathscr{T}}, \mathrm{p}_{\mathscr{T}}\right)\right)\right\|_{\tilde{\sim}}^{2}{ }_{\tilde{L}}^{2}(K) \\
& =\sum_{K \in \mathscr{T}}\left\|\nabla \mathscr{L}\left(\mathbf{u}-\mathbf{u}_{\mathscr{T}}, \mathrm{p}-\mathrm{p}_{\mathscr{T}}\right)\right\|_{\tilde{\sim}^{2}(K)}^{2} \\
& \leq C\left(\left\|\nabla\left(\mathbf{u}-\mathbf{u}_{\mathscr{T}}\right)\right\|_{\underline{\sim}^{2}(\Omega)}^{2}+\sum_{\gamma \in \mathcal{F}_{I}} \tau_{\gamma}\left\|\llbracket \mathrm{p}-\mathrm{p}_{\mathscr{T}} \mathbb{1}_{\gamma}\right\|_{L^{2}(\gamma)}^{2}\right),
\end{aligned}
$$

which proves the result.

As it has been stated earlier, $\mathscr{L}\left(\mathbf{u}_{\mathscr{T}}, \mathrm{p}_{\mathscr{T}}\right)$ belongs to $\boldsymbol{H}_{0}(\mathbf{d i v}, \Omega)$, but its tangential component at the boundary is not zero. So, it does not satisfy the non-slip boundary condition exactly. Fortunately, in the next result we show that its tangential component converges to zero with a higher rate than the method. For simplicity we limit ourselves to showing the error in the violation of the boundary condition on $\mathbf{u}$, but the proof can be extended without major difficulty to show an analogous result for the jump of the tangential component of the velocity $\mathscr{L}\left(\mathbf{u}_{\mathscr{T}}, \mathrm{p}_{\mathscr{T}}\right)$, in all the interior edges. 
Lemma 6. Let, for every $\gamma \in \mathcal{F} \cap \Gamma, \boldsymbol{t}_{\gamma}$ be any unit vector parallel to $\gamma$. Then, there exists $C>0$, independent of $h$, such that

$$
\left(\sum_{\gamma \in \mathcal{F} \cap \Gamma}\left\|\mathscr{L}\left(\mathbf{u}_{\mathscr{T}}, \mathrm{p}_{\mathscr{T}}\right) \cdot \boldsymbol{t}_{\gamma}\right\|_{L^{2}(\gamma)}^{2}\right)^{\frac{1}{2}} \leq C h^{\frac{1}{2}}\left(\sum_{\gamma \in \mathcal{F}_{I}} \tau_{\gamma}\left\|\llbracket \mathrm{p}-\mathrm{p}_{\mathscr{T}}\right\|_{\gamma} \|_{L^{2}(\gamma)}^{2}\right)^{1 / 2} .
$$

Proof. To every face (edge) $\gamma \in \mathcal{F} \cap \Gamma$ we associate a unique simplex $K_{\gamma} \in \mathscr{T}$ such that $\gamma \in \mathcal{F}_{K_{\gamma}}$. We then notice that, since $\mathbf{u}_{\mathscr{T}}$ vanishes at the boundary, then

$$
\left.\mathscr{L}\left(\mathbf{u}_{\mathscr{T}}, \mathrm{p}_{\mathscr{T}}\right)\right|_{K_{\gamma}} \cdot \boldsymbol{t}_{\gamma}=\sum_{\gamma^{\prime} \in \mathcal{F}_{K_{\gamma}} \cap \mathcal{F}_{I}} \tau_{\gamma^{\prime}} \llbracket p_{\mathscr{T}} \rrbracket_{\gamma^{\prime}} \boldsymbol{\varphi}_{\gamma^{\prime}} \cdot \boldsymbol{t}_{\gamma},
$$

where $\varphi_{\gamma^{\prime}}$ is the Raviart-Thomas basis function associated to $\gamma^{\prime}$, defined in (1). Moreover, thanks to the mesh regularity we have $\left|\boldsymbol{\varphi}_{\gamma^{\prime}} \cdot \boldsymbol{t}_{\gamma}\right| \leq C$, where $C$ does not depend on $h$. This, together with the mesh regularity and the definition of $\tau_{\gamma^{\prime}}$, imply that

$$
\left\|\mathscr{L}\left(\mathbf{u}_{\mathscr{T}}, \mathrm{p}_{\mathscr{T}}\right) \cdot \boldsymbol{t}_{\gamma}\right\|_{L^{2}(\gamma)}^{2} \leq C \sum_{\gamma^{\prime} \in \mathcal{F}_{K_{\gamma}} \cap \mathcal{F}_{I}} \tau_{\gamma^{\prime}}^{2} \llbracket p_{\mathscr{T}} \mathbb{1}_{\gamma^{\prime}}^{2}\left|\gamma^{\prime}\right| \leq C h \sum_{\gamma^{\prime} \in \mathcal{F}_{K_{\gamma}} \cap \mathcal{F}_{I}} \tau_{\gamma^{\prime}}\left\|\llbracket p p_{\mathscr{T}}\right\| \|_{L^{2}\left(\gamma^{\prime}\right)}^{2} .
$$

The proof then follows adding over all $\gamma \in \mathcal{F} \cap \Gamma$.

Remark 5. We finish this section by mentioning that a qualitatively similar error estimate to the one obtained in this section can be derived by using an alternative error analysis, this time based on the theory developed in [30] (see also [25] for its application to the Navier-Stokes equation), can be carried out. Nevertheless, in that case, the error estimates necessarily need the mesh to be fine enough, and the assumption of smallness of the data and solutions would also be needed.

\section{Numerical experiments}

In this section we conduct a series of numerical examples that illustrate the performance of the proposed low-order stabilized finite element scheme. These have been carried out with the help of a home built $\mathrm{C}++$ code. All matrices, right hand sides, and approximation errors are computed by a quadrature formula which is exact for polynomials of degree 19. All linear systems were solved using the multifrontal massively parallel sparse direct solver (MUMPS) $[31,32]$.

To implement our method, first we make the following choices for stabilizing terms:

$$
\forall \boldsymbol{\xi}_{\mathscr{T}}, \boldsymbol{\zeta}_{\mathscr{T}} \in \boldsymbol{V}(\mathscr{T}): \quad \mathcal{S}_{F}\left(\boldsymbol{\xi}_{\mathscr{T}}, \boldsymbol{\zeta}_{\mathscr{T}}\right)=\delta\left\{\begin{array}{cl}
\sum_{K \in \mathscr{T}} h_{K} \int_{K} \nabla \cdot \boldsymbol{\xi}_{\mathscr{T}} \nabla \cdot \boldsymbol{\zeta}_{\mathscr{T}} & \begin{array}{c}
0 \text {-type, } \\
\text { div-div-type, }
\end{array} \\
\sum_{\gamma \in \mathcal{F}_{I}} h_{\gamma}^{2} \int_{\gamma} \llbracket \partial_{\boldsymbol{n}} \boldsymbol{\xi}_{\mathscr{T}} \rrbracket_{\gamma} \llbracket \partial_{\boldsymbol{n}} \boldsymbol{\zeta}_{\mathscr{T}} \rrbracket_{\gamma} & \text { CIP-type, }
\end{array}\right.
$$

where $\delta>0$ and, for any $\gamma \in \mathcal{F}_{K} \cap \mathcal{F}_{K^{\prime}}$,

$$
\llbracket \partial_{\boldsymbol{n}} \boldsymbol{\xi}_{\mathscr{T}} \rrbracket_{\gamma}=\nabla \boldsymbol{\xi}_{\mathscr{T} \mid K} \boldsymbol{n}_{\gamma}^{K}+\nabla \boldsymbol{\xi}_{\mathscr{T} \mid K^{\prime}} \boldsymbol{n}_{\gamma}^{K^{\prime}}
$$

Clearly, the first choice (no stabilization) satisfies the assumptions automatically. For the other two choices, we notice that for the div-div-type stabilization, such properties can be obtained by using a Cauchy-Schwarz inequality and (3). For a CIP-type choice, the results follows by using again a Cauchy-Schwarz inequality and the results appearing in [29].

For the stabilization term for the temperature equation, we consider the following choices:

$$
\forall \theta_{\mathscr{T}}, \psi_{\mathscr{T}} \in V(\mathscr{T}): \quad \mathcal{S}_{T}\left(\theta_{\mathscr{T}}, \psi_{\mathscr{T}}\right)=\tilde{\delta}\left\{\begin{array}{cl}
0 & 0 \text {-type, } \\
\sum_{\gamma \in \mathcal{F}_{I}} h_{\gamma}^{2} \int_{\gamma} \llbracket \partial_{\boldsymbol{n}} \theta_{\mathscr{T}} \rrbracket_{\gamma} \llbracket \partial_{\boldsymbol{n}} \psi_{\mathscr{T}} \rrbracket_{\gamma}, & \text { CIP-type, }
\end{array}\right.
$$


where $\tilde{\delta}>0$, and $\llbracket \partial_{\boldsymbol{n}} \theta_{\mathscr{T}} \rrbracket_{\gamma}$ is defined as in (75). Here, assumptions (19)-(20) can be obtained by using the CauchySchwarz inequality and the results appearing in [29].

Remark 6. In the above stabilizing forms, the parameters $\delta, \tilde{\delta}$ are just supposed to be positive constants. We have chosen them to be equal to 1 in our numerical experiments, and that choice has proven sufficient. Now, from a dimensional point of view, these two parameters should depend on the convective field. So, they should be the product of a dimensionless constant and some norm of the convective field, as it is presented in, e.g., [33]. We have avoided to make this dependence explicit in our analysis (and our numerical experiments) not to introduce one further nonlinearity in these terms.

The convergence will be measured using the following norms:

$$
\begin{gathered}
\|\xi\|_{\mathcal{N}(\Omega)}^{2}:=\varepsilon_{1}\|\nabla \xi\|_{L^{2^{(}(\Omega)}}^{2}+\|\xi\|_{L^{2}(\Omega)}^{2}, \quad\|\theta\|_{\mathcal{T}(\Omega)}^{2}:=\kappa_{1}\|\nabla \theta\|_{L^{2}(\Omega)}^{2}+\|\theta\|_{L^{2}(\Omega)}^{2}, \\
\|(\xi, \phi, \theta)\|_{\mathscr{T}}^{2}:=\|(\xi, \phi)\|_{\mathcal{N}(\mathscr{T})}^{2}+\|\theta\|_{\mathcal{T}(\mathscr{T})}^{2} .
\end{gathered}
$$

We also introduce the error for the divergence-free discrete velocity:

$$
e^{\mathbf{u}, \mathrm{p}}:=\mathbf{u}-\mathscr{L}\left(\mathbf{u}_{\mathscr{T}}, \mathrm{p}_{\mathscr{T}}\right)
$$

In order to obtain analytical solutions of the model problem, we will consider the following slightly generalized situation, where non-homogeneous right-hand sides are considered:

$$
\left\{\begin{array}{rlll}
-\operatorname{div}(\varepsilon(\mathrm{t}) \nabla \mathbf{u})+\mathbf{u} \cdot(\nabla \mathbf{u})+\nabla \mathrm{p}-\mathbf{g t} & =\mathbf{f} & \text { in } \Omega, \\
\operatorname{div} \mathbf{u} & =0 & & \text { in } \Omega, \\
-\operatorname{div}(\kappa(\mathrm{t}) \nabla \mathrm{t})+\mathbf{u} \cdot \nabla \mathrm{t} & =\mathrm{h} & & \text { in } \Omega, \\
\mathbf{u} & =\mathbf{0} & \text { on } \Gamma, \\
\mathrm{t} & =\mathrm{t}_{D} & & \text { on } \Gamma,
\end{array}\right.
$$

where $\mathbf{f} \in \boldsymbol{L}^{2}(\Omega)$ and $\mathrm{h} \in L^{2}(\Omega)$.

The discrete problem (15) is solved using a damped fixed point algorithm. More precisely, the algorithm is started with any initial guess $\mathrm{t}_{\mathscr{T}}^{0}$ that satisfies the boundary conditions. Then, an initial guess for the velocity and pressure, $\left(\mathbf{u}_{\mathscr{T}}^{0}, \mathrm{p}_{\mathscr{T}}^{0}\right)$, is computed solving a discrete Stokes problem, and the divergence-free field $\mathscr{L}\left(\mathbf{u}_{\mathscr{T}}^{0}, \mathrm{p}_{\mathscr{T}}^{0}\right)$ is built. Then, for any $n=0,1,2, \ldots$, the intermediate step $(\tilde{\mathbf{u}}, \tilde{p}, \tilde{\mathrm{t}}) \in V_{0}(\mathscr{T}) \times Q(\mathscr{T}) \times V(\mathscr{T})$ is computed solving the following decoupled problems: $\tilde{\mathrm{t}}_{\mid \Gamma}=i_{h}\left(\mathrm{t}_{D}\right)$, and

$$
\left\{\begin{aligned}
\mathcal{A}_{F}\left(\mathrm{t}_{\mathscr{T}}^{n} ; \tilde{\mathbf{u}}, \boldsymbol{\xi}_{\mathscr{T}}\right)+\mathcal{C}_{F}\left(\mathscr{L}\left(\mathbf{u}_{\mathscr{T}}^{n}, \mathrm{p}_{\mathscr{T}}^{n}\right) ; \tilde{\mathbf{u}}, \boldsymbol{\xi}_{\mathscr{T}}\right)+\mathcal{S}_{F}\left(\tilde{\mathbf{u}}, \boldsymbol{\xi}_{\mathscr{T}}\right)-\mathcal{B}_{F}\left(\boldsymbol{\xi}_{\mathscr{T}}, \tilde{\mathrm{p}}\right)-\mathcal{G}_{F}\left(\mathrm{t}_{\mathscr{T}}^{n}, \boldsymbol{\xi}_{\mathscr{T}}\right) & =\int_{\Omega} \mathbf{f} \cdot \boldsymbol{\xi}_{\mathscr{T}}, \\
\mathcal{B}_{F}\left(\tilde{\mathbf{u}}, \phi_{\mathscr{T}}\right)+\sum_{\gamma \in \mathcal{F}_{I}} \tau_{\gamma} \int_{\gamma} \llbracket \tilde{\mathrm{p}} \rrbracket_{\gamma} \llbracket \phi_{\mathscr{T}} \rrbracket_{\gamma} & =0, \\
\mathcal{A}_{T}\left(\mathrm{t}_{\mathscr{T}}^{n} ; \tilde{\mathrm{t}}, \psi_{\mathscr{T}}\right)+C_{T}\left(\mathscr{L}\left(\mathbf{u}_{\mathscr{T}}^{n}, \mathrm{p}_{\mathscr{T}}^{n}\right) ; \tilde{\mathrm{t}}, \psi_{\mathscr{T}}\right)+\mathcal{S}_{T}\left(\tilde{\mathrm{t}}, \psi_{\mathscr{T}}\right) & =\int_{\Omega} \mathrm{h} \psi_{\mathscr{T}},
\end{aligned}\right.
$$

for all $\left(\xi_{\mathscr{T}}, \phi_{\mathscr{T}}\right) \in V_{0}(\mathscr{T}) \times Q(\mathscr{T})$ and all $\psi_{\mathscr{T}} \in V_{0}(\mathscr{T})$, respectively. Then, for $\omega \in(0,1]$, the next iterate is built as follows

$$
\left(\begin{array}{c}
\mathbf{u}_{\mathscr{T}}^{n+1} \\
\mathbf{p}_{\mathscr{T}}^{n+1} \\
\mathbf{t}_{\mathscr{T}}^{n+1}
\end{array}\right)=\left(\begin{array}{c}
\mathbf{u}_{\mathscr{T}}^{n} \\
\mathbf{p}_{\mathscr{T}}^{n} \\
\mathbf{t}_{\mathscr{T}}^{n}
\end{array}\right)+\omega\left(\begin{array}{c}
\tilde{\mathbf{u}}-\mathbf{u}_{\mathscr{T}}^{n} \\
\tilde{p^{\prime}}-\mathbf{p}_{\mathscr{T}}^{n} \\
\tilde{\mathrm{t}}-\mathbf{t}_{\mathscr{T}}^{n}
\end{array}\right) .
$$

For the cases in which the Rayleigh number is large (see Examples 2 and 3, below), a parameter continuation strategy has been used as well.

Finally, when measuring convergence rates with respect the total number of degreees of freedom, we adopt the notation $\operatorname{Ndof}=\operatorname{dim}\left(\boldsymbol{V}_{0}(\mathscr{T})\right)+\operatorname{dim}(Q(\mathscr{T}))+\operatorname{dim}(V(\mathscr{T}))$. 
Example 1 (a problem with an analytical interior temperature layer): We consider $\Omega=(0,1)^{2}$, and we choose $\mathbf{f}$, $\mathrm{h}$ and $\mathrm{t}_{D}$ such that

$$
\begin{gathered}
\mathbf{u}(x, y)=70 \operatorname{curl}(x y(1-x)(1-y))^{2}, \quad \mathrm{p}(x, y)=x^{2} y^{2}(1-x)(1-y)-1 / 144, \quad \varepsilon(\mathrm{t})=\varepsilon=1, \\
\mathrm{t}(x, y)=x y(1-x)(1-y) \tan ^{-1}((x-0.5) / \kappa), \quad \kappa(\mathrm{t})=\kappa=10^{-4} .
\end{gathered}
$$

For this problem we have solved (78) and (79) on a fixed mesh, containing 82691 degrees of freedom. We have chosen to fix a 0 -type stabilization as $\mathcal{S}_{F}=0$ in (74), and consider the two alternatives for $\mathcal{S}_{T}$ given in (76).

The approximate temperatures are depicted in Figure 2. We can observe in there that, as expected, not including the stabilizing term $\mathcal{S}_{T}$ leads to a highly oscillatory solution. So, from now on, we always consider $\mathcal{S}_{T}$ to be the CIP-type considered in (76).

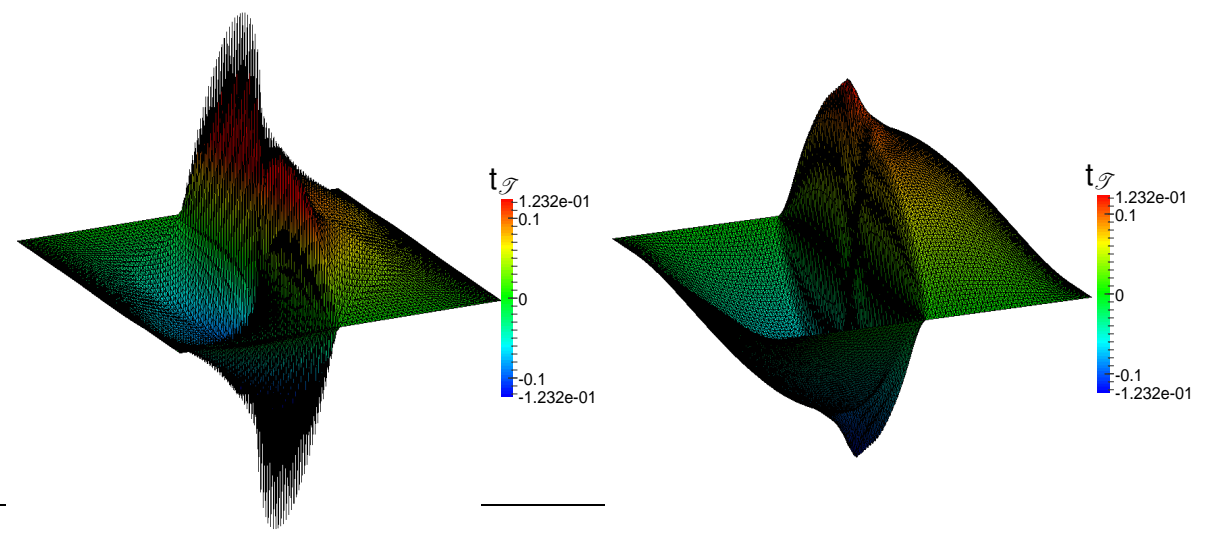

Figure 2: Finite element solutions for Example 1: approximation of the temperature variable by using a 0 -type stabilization in $\mathcal{S}_{T}$ (left), and a CIP-type stabilization in $\mathcal{S}_{T}$ (right), on a mesh with 32768 elements.

Remark 7. In addition to the result just showed, in our experience adding a non-zero stabilizing term $\mathcal{S}_{T}$ is also of importance from the linear algebra perspective. In fact, we observed that not including this stabilizing term led, in some cases, to non-convergence of the fixed point algorithm.

Example 2 (a problem with an analytical solution and temperature-dependent viscosity and thermal conductivity): We consider $\Omega=(-1,1)^{2}$, and we choose $\mathbf{f}, \mathrm{h}$ and $\mathrm{t}_{D}$ such that

$$
\mathbf{u}(x, y)=\left(\begin{array}{l}
\sin (\pi y) \\
\sin (\pi x)
\end{array}\right), \quad \mathrm{p}(x, y)=\sin (x y), \quad \mathrm{t}(x, y)=5 \cos (\pi x y), \quad \varepsilon(\mathrm{t})=e^{-\mathrm{t}}, \quad \kappa(\mathrm{t})=e^{\mathrm{t}} .
$$

In this case we have that $\varepsilon_{1}=e^{-5}, \varepsilon_{2}=e^{5}, \kappa_{1}=e^{-5}$ and $\kappa_{2}=e^{5}$. Due to the ranges of values for $\varepsilon$ and $\kappa$, this problem has regions in which convection dominates, and others in which the problem is diffusion dominated.

We have solved (78) and (79) in a sequence of meshes refined uniformly. We computed the errors for all variables by using the three possible choices for stabilizing form $\mathcal{S}_{F}$ in (74), and a CIP-type stabilization for $\mathcal{S}_{T}$. The errors are depicted, for each norm and the total norm in Figure 3. We have depicted, for each error, the three curves obtained by each one of the three choices for $\mathcal{S}_{F}$ form (74). We can observe that, in addition to converging optimally, for all norms the three curves are almost indistinguishable. This has also been the case for subsequent numerical experiments, and hints that the modification of the convective term has a stabilizing effect by itself. In Figure 4 we plot the discrete solution obtained for the finest mesh of the sequence, for the case $\mathcal{S}_{F}=\mathbf{0}$ and the CIP-type stabilization for $\mathcal{S}_{T}$.

Next, to assess the difference between the discrete solutions $\mathbf{u}_{\mathscr{T}}$ and $\mathscr{L}\left(\mathbf{u}_{\mathscr{T}}, \mathrm{p}_{\mathscr{T}}\right)$ we have computed the divergence of both these fields over a sequence of meshes. The results are reported in Table 1, where we can observe that while the divergence of $\mathbf{u}_{\mathscr{T}}$ is significant, $\mathscr{L}\left(\mathbf{u}_{\mathscr{T}}, \mathrm{p}_{\mathscr{T}}\right)$ is divergence-free up to machine precision for all meshes, which confirms Theorem 1. It is interesting to notice that for this example the impact of the div-div term is negligible in the value of the divergence of $\mathbf{u}_{\mathscr{T}}$. 


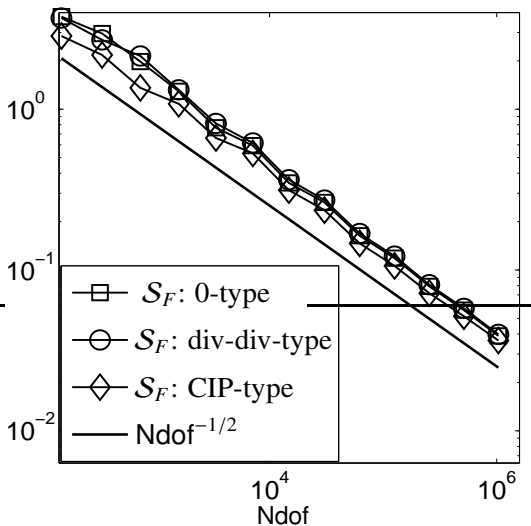

(a)

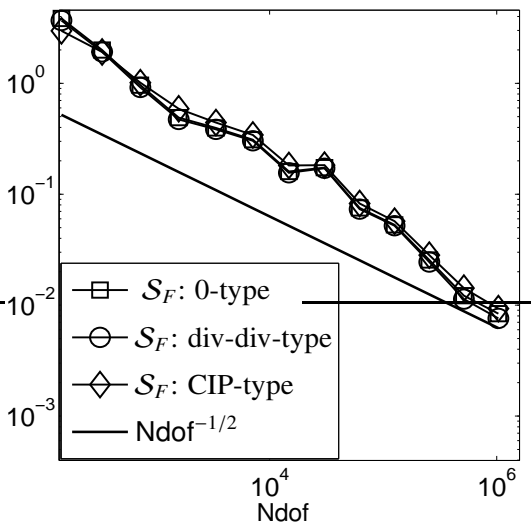

(d)

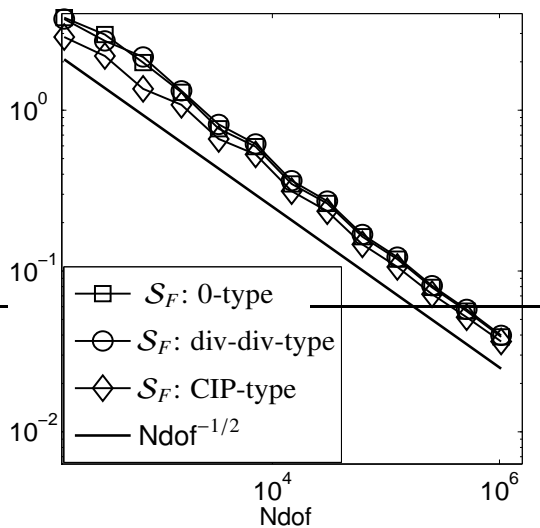

(b)

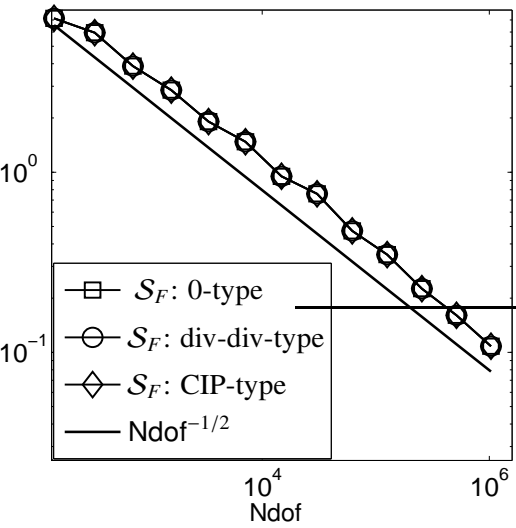

(e)

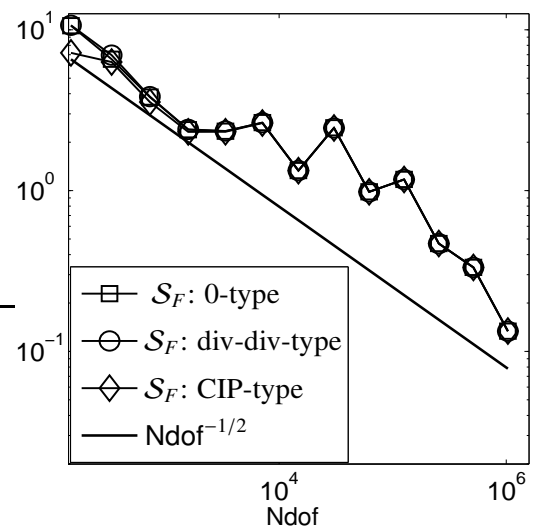

(c)

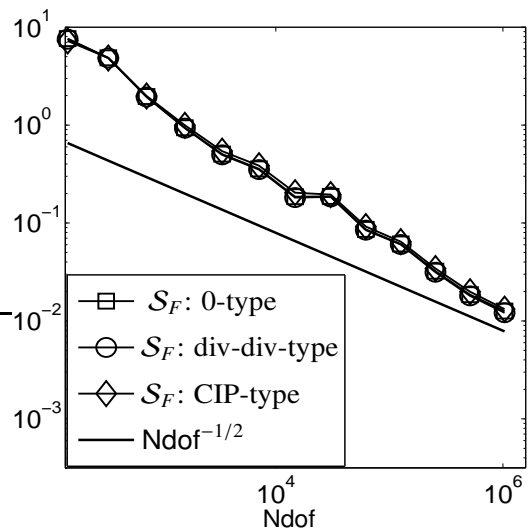

(f)

Figure 3: Convergence rates for Example 2 with respect to the total number of degrees of freedom for every choice of the stabilization term $\mathcal{S}_{F}$ : (a) shows the convergence of $\left\|\nabla \boldsymbol{e}^{u}\right\|_{L^{2}(\Omega)}$ and (b) shows the convergence of $\left\|\nabla \boldsymbol{e}^{u, p}\right\|_{\boldsymbol{L}^{2}(\Omega)}$, (c) shows the convergence of $\left\|e^{p}\right\|_{L^{2}(\Omega)}$, (d) shows the convergence of $\left(\sum \tau_{\gamma} \|\left[e^{\mathrm{p}} \rrbracket_{\gamma} \|_{L^{2}(\gamma)}^{2}\right)^{1 / 2}\right.$, (e) shows the convergence of $\left\|\nabla e^{t}\right\|_{L^{2}(\Omega)}$ and (f) shows the total convergence of the discrete solution in the $\left\|\left(\boldsymbol{e}^{u}, e^{p}, e^{t}\right)\right\|_{\mathscr{T}}$ norm. In all the different norms we can observe optimal convergence rates.

\begin{tabular}{|c|c|c|c|c|c|c|}
\hline & \multicolumn{2}{|c|}{$\mathcal{S}_{F}(\boldsymbol{\xi}, \zeta)=0$} & \multicolumn{2}{|c|}{$\mathcal{S}_{F}(\xi, \zeta)=\sum_{K \in \mathscr{T}} h_{K} \int_{K} \nabla \cdot \xi \nabla \cdot \zeta$} & \multicolumn{2}{|c|}{$\mathcal{S}_{F}(\xi, \zeta)=\sum_{\gamma \in \mathcal{F}_{I}} h_{\gamma}^{2} \int_{\gamma} \llbracket \partial_{\boldsymbol{n}} \xi_{\mathscr{T}} \mathbb{1}_{\gamma} \llbracket \partial_{\boldsymbol{n}} \zeta_{\mathscr{T}} \mathbb{1}_{\gamma}$} \\
\hline Ndof & $\max _{K \in \mathscr{T}}|\operatorname{div} \mathbf{u} \mathscr{T}|_{K}$ & $\max _{K \in \mathscr{T}}\left|\operatorname{div} \mathscr{L}\left(\mathbf{u}_{\mathscr{T}}, \mathrm{p}_{\mathscr{T}}\right)\right|_{K} \mid$ & $\max _{K \in \mathscr{T}}\left|\operatorname{div} \mathbf{u}_{\mathscr{T}}\right|_{K}$ & $\max _{K \in \mathscr{T}}\left|\operatorname{div} \mathscr{L}\left(\mathbf{u}_{\mathscr{T}}, \mathrm{p}_{\mathscr{T}}\right)\right|_{K} \mid$ & $\max _{K \in \mathscr{T}}|\operatorname{div} \mathbf{u} \mathscr{T}|_{K}$ & $\max _{K \in \mathscr{T}}\left|\operatorname{div} \mathscr{L}\left(\mathbf{u}_{\mathscr{T}}, \mathrm{p}_{\mathscr{T}}\right)\right|_{K} \mid$ \\
\hline 147 & 0.147 & $1.39 \mathrm{e}-16$ & 0.141 & $2.01 \mathrm{e}-16$ & 0.114 & $1.8 \mathrm{e}-16$ \\
\hline 335 & 0.0533 & $1.8 \mathrm{e}-16$ & 0.042 & $1.11 \mathrm{e}-16$ & 0.0376 & $2.5 \mathrm{e}-16$ \\
\hline 727 & 0.0122 & $1.67 \mathrm{e}-16$ & 0.012 & $1.48 \mathrm{e}-16$ & 0.0118 & $2.78 \mathrm{e}-16$ \\
\hline 1583 & 0.00393 & $5.72 \mathrm{e}-16$ & 0.00338 & $9.02 \mathrm{e}-17$ & 0.00413 & $2.5 \mathrm{e}-16$ \\
\hline 3367 & 0.00164 & $1.32 \mathrm{e}-16$ & 0.00158 & $1.39 \mathrm{e}-16$ & 0.00169 & $1.87 \mathrm{e}-16$ \\
\hline 7119 & 0.000554 & $1.67 \mathrm{e}-16$ & 0.000549 & $1.18 \mathrm{e}-16$ & 0.000659 & $1.18 \mathrm{e}-16$ \\
\hline 14767 & 0.000178 & $1.01 \mathrm{e}-16$ & 0.000175 & $7.98 \mathrm{e}-17$ & 0.000178 & $1.01 \mathrm{e}-16$ \\
\hline 30411 & $6.87 \mathrm{e}-05$ & $1.7 \mathrm{e}-16$ & $6.82 \mathrm{e}-05$ & $5.9 \mathrm{e}-17$ & $7.6 e-05$ & $1.08 \mathrm{e}-16$ \\
\hline 62027 & $2 \mathrm{e}-05$ & $5.9 \mathrm{e}-17$ & $1.93 \mathrm{e}-05$ & $5.9 \mathrm{e}-17$ & $2.08 \mathrm{e}-05$ & $1.06 \mathrm{e}-16$ \\
\hline 125995 & $6.36 \mathrm{e}-06$ & $8.63 \mathrm{e}-17$ & $5.33 \mathrm{e}-06$ & $3.64 \mathrm{e}-17$ & $7.73 e-06$ & $1.18 \mathrm{e}-16$ \\
\hline 254627 & $2.58 \mathrm{e}-06$ & $7.63 \mathrm{e}-17$ & $2.32 \mathrm{e}-06$ & $2.95 \mathrm{e}-17$ & $2.56 \mathrm{e}-06$ & $9.97 \mathrm{e}-17$ \\
\hline 513403 & $8.72 \mathrm{e}-07$ & $1.2 \mathrm{e}-16$ & $6.76 \mathrm{e}-07$ & $3.12 \mathrm{e}-17$ & $9.38 \mathrm{e}-07$ & $6.14 \mathrm{e}-17$ \\
\hline 1032387 & $3.24 \mathrm{e}-07$ & $4.16 \mathrm{e}-17$ & $2.93 \mathrm{e}-07$ & $2.17 \mathrm{e}-17$ & $3.23 \mathrm{e}-7$ & $7.46 \mathrm{e}-17$ \\
\hline
\end{tabular}

Table 1: Maximum absolute value of the divergence of the solution $\mathbf{u}_{\mathscr{T}}$ and its divergence-free counterpart $\mathscr{L}\left(\mathbf{u}_{\mathscr{T}}, \mathrm{p}_{\mathscr{T}}\right)$ for Example 2 , computed with all the different choices for the stabilizing term $\mathcal{S}_{F}$ and fixing a CIP-type stabilization for $\mathcal{S}_{T}$. 


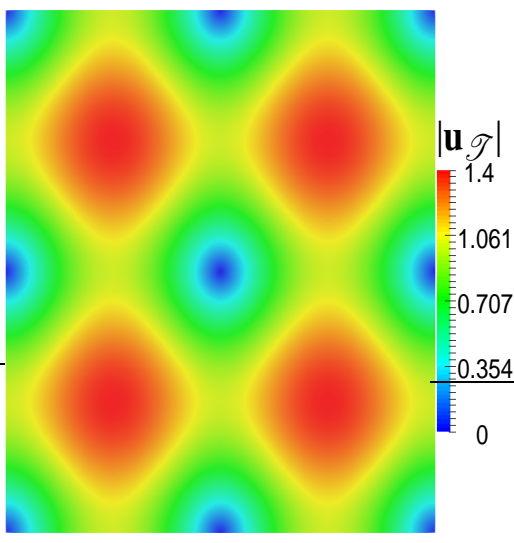

(a)

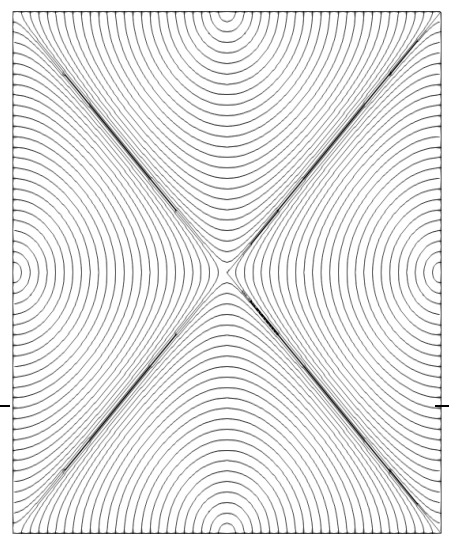

(d)

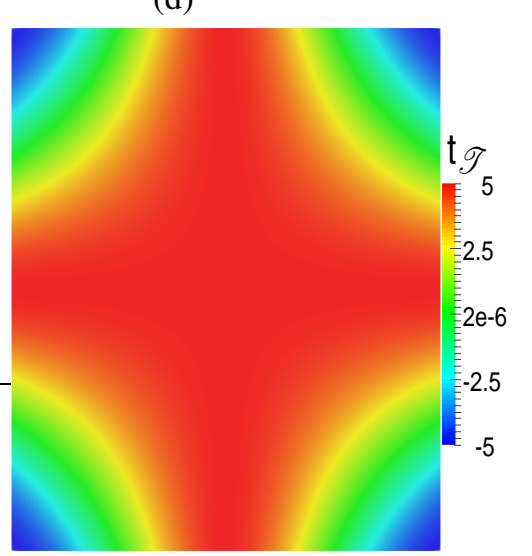

(g)

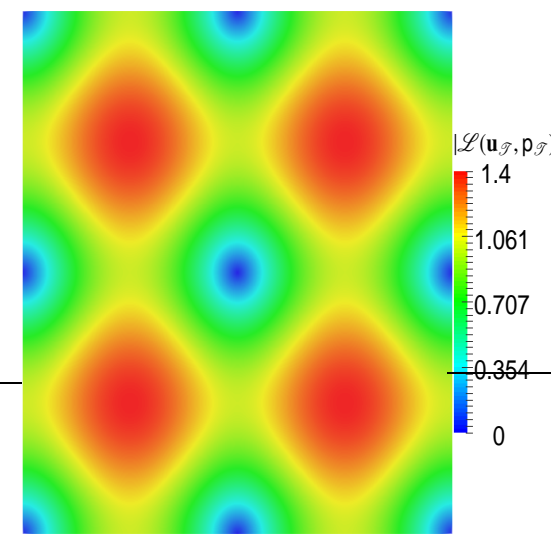

(b)

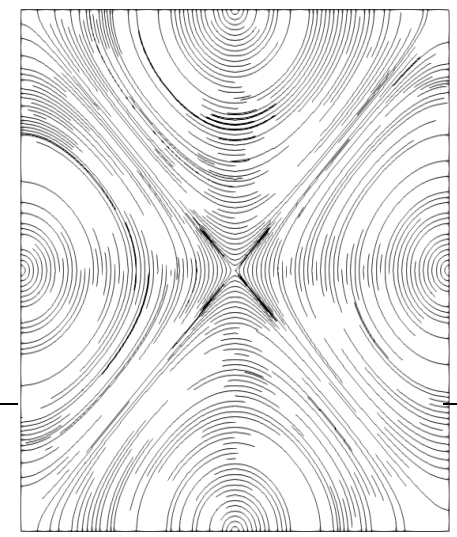

(e)

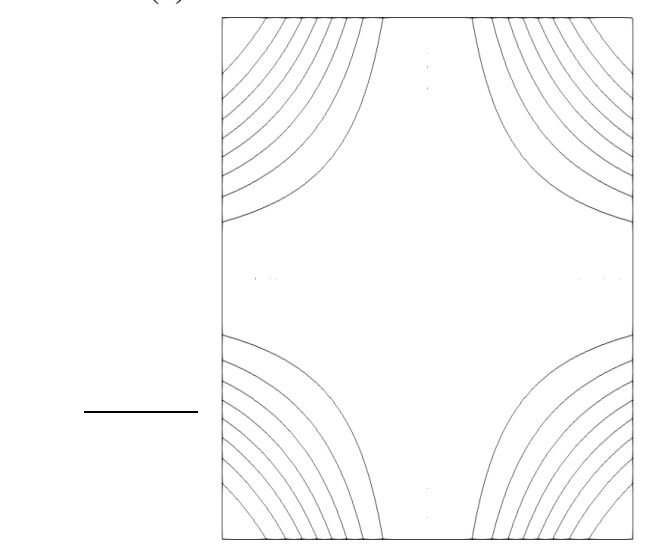

(h)

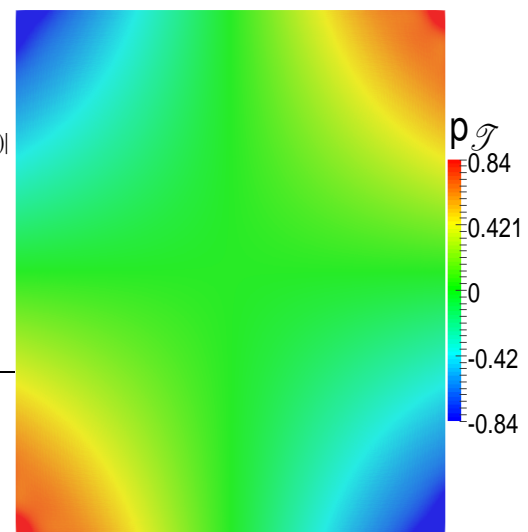

(c)

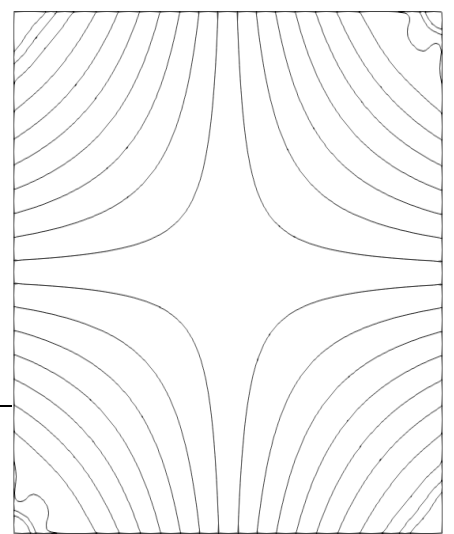

(f)
$P_{0.84}$

0.421 


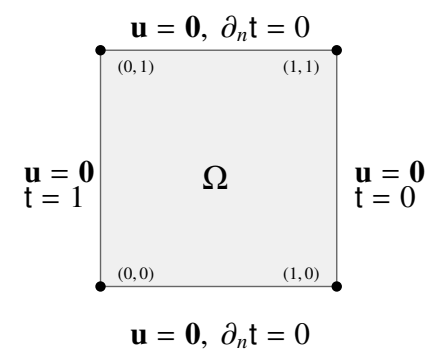

Figure 5: Boundary conditions for Example 3 and 4.

Example 3 (Differentially heated cavity flow: the Stokes case): This example has been considered previously in [34]. We set $\Omega=(0,1)^{2}$, and consider the following problem

$$
\left\{\begin{aligned}
-\Delta \mathbf{u}+\nabla \mathrm{p} & =(0 R a)^{T} \mathrm{t} & & \text { in } \Omega, \\
\operatorname{div} \mathbf{u} & =0 & & \text { in } \Omega, \\
-\Delta \mathrm{t}+\mathbf{u} \cdot \nabla \mathrm{t} & =0 & & \text { in } \Omega,
\end{aligned}\right.
$$

where the boundary conditions are given in Figure 5. Here $R a$ is Rayleigh's number, which is associated with the heat transfer inside the fluid; a low value for $R a$ implies that the conduction transfer is dominant, a high value $(R a>1000)$ implies that the convection transfer is dominant. For our computations, we have chosen a range of values and report the results for $R a=10^{4}$ and $R a=10^{6}$, and we have performed computations using the three possible choices given in (74) for $\mathcal{S}_{F}$, and the CIP-type in (76) for $\mathcal{S}_{T}$.

First, for $R a=10^{4}$, the divergences of both $\mathbf{u}_{\mathscr{T}}$ and $\mathscr{L}\left(\mathbf{u}_{\mathscr{T}}, \mathrm{p}_{\mathscr{T}}\right)$ are given in Table 2, comparing results for the mentioned three choices for $\mathcal{S}_{F}$, where the same conclusions as for the previous example can be drawn. For $R a=10^{6}$, we just show results for the 0-type stabilization for $\mathcal{S}_{F}$ and a CIP-type for $\mathcal{S}_{T}$; a highly resolved solution is depicted in Figure 6 (d.2), where we depict the streamlines of the velocity. This solution is very similar to the one presented in [34, Fig.9]. In Figure 6 (a.1) to (h.1) we depict the discrete solution, including isovalues for the temperature and streamlines for both the discrete velocities $\mathbf{u}_{\mathscr{T}}$ and $\mathscr{L}\left(\mathbf{u}_{\mathscr{T}}, \mathrm{p}_{\mathscr{T}}\right)$, using a coarse mesh with 16384 elements (41347 degrees of freedom). Then, in Figure 6 (a.2) to (h.2), we depict the same plots, but using a very fine mesh, with 524288 elements (1313795 degrees of freedom). The pressure and temperature are similar for both meshes, hinting to a robustness of the method. The discrete velocities, on the other hand, present a striking behavior. When comparing the streamlines depicted in Figure 6 (c.1) and (d.1), we observe that the streamlines of the non-divergence-free field $\mathbf{u}_{\mathscr{T}}$ differ from the resolved one by a significant margin, while the streamlines of $\mathscr{L}\left(\mathbf{u}_{\mathscr{T}}, \mathrm{p}_{\mathscr{T}}\right)$ are very close to the resolved ones even for the very coarse mesh. This shows the improvement given by non-conforming, but divergencefree, velocity given by $\mathscr{L}\left(\mathbf{u}_{\mathscr{T}}, \mathrm{p}_{\mathscr{T}}\right)$ with respect to the conforming polynomial velocity $\mathbf{u}_{\mathscr{T}}$.

Example 4 (Differentially heated cavity flow: the Navier-Stokes case): We set $\Omega=(0,1)^{2}$, and consider the following problem

$$
\left\{\begin{aligned}
-P r \Delta \mathbf{u}+(\mathbf{u} \cdot \nabla) \mathbf{u}+\nabla \mathrm{p} & =(0 \operatorname{Pr} R a)^{T} \mathrm{t} & & \text { in } \Omega, \\
\operatorname{div} \mathbf{u} & =0 & & \text { in } \Omega, \\
-\Delta \mathrm{t}+\mathbf{u} \cdot \nabla \mathrm{t} & =0 & & \text { in } \Omega,
\end{aligned}\right.
$$

where the boundary conditions are given once again in Figure 5. This case is a standard benchmark for which numerous numerical studies have been carried out (see, e .g, $[10,7,8,9,11,12])$. Here, $R a$ again denotes the Rayleigh's number, and $\operatorname{Pr}$ is Prandtl's number, defined as $\varepsilon_{r} / \kappa_{r}$, where $\varepsilon_{r}$ and $\kappa_{r}$ are a reference viscosity and thermal conductivity, respectively. In this case, we use the value for the air $\operatorname{Pr}=0.71$, as suggested in the literature. We mention that for this example it is well-known that solutions exist in the range of Rayleigh numbers up to $R a \approx 2.0 \cdot 10^{8}$ (see [35] for details). 
We have solved this problem for Rayleigh numbers ranging from $R a=10^{3}$ to $R a=10^{7}$. First, for $R a=10^{4}$ we report the values of the maximum divergence of $\mathbf{u}_{\mathscr{T}}$ and $\mathscr{L}\left(\mathbf{u}_{\mathscr{T}}, \mathrm{p}_{\mathscr{T}}\right)$ in Table 3 , where we compare the values obtained for the other three posssible choices for $\mathcal{S}_{F}$. Once again the divergence of $\mathbf{u}_{\mathscr{T}}$ is significant, while the field $\mathscr{L}\left(\mathbf{u}_{\mathscr{T}}, \mathrm{p}_{\mathscr{T}}\right)$ is divergence-free. Once again, we notice that the addition of a non-zero stabilization for $\mathcal{S}_{F}$, in particular the div-div form of it, has virtually no impact in the divergence of $\mathbf{u}_{\mathscr{T}}$. Then, from now on we only report the results for 0-type stablization from (74) for $\mathcal{S}_{F}$, and the CIP-type in (76) for $\mathcal{S}_{T}$. Next, we carry out the same analysis as in the previous example, and compute the discrete solution for $R a=10^{6}$ and $R a=10^{7}$. We use the same order as in the Figure 6, and in Figure 7 we show the corresponding results for $R a=10^{6}$, while the ones $R a=10^{7}$ are depicted in Figure 8. The same comments as in the previous example are valid. It is important to state that the divergence-free solution given by $\mathscr{L}\left(\mathbf{u}_{\mathscr{T}}, \mathrm{p}_{\mathscr{T}}\right)$ recovers, even for coarse meshes, physical features of the exact solution which are not present in the conforming velocity $\mathbf{u}_{\mathscr{T}}$. This is especially noticieable for $R a=10^{7}$, where the results for the coarse mesh and the fine mesh are very similar for $\mathscr{L}\left(\mathbf{u}_{\mathscr{T}}, \mathrm{p}_{\mathscr{T}}\right)$, while the streamlines of $\mathbf{u}_{\mathscr{T}}$ would need a much finer mesh to show the correct behaviour. We stress the fact that the plots depicted in Figures 7 and 8 are obtained using $\mathcal{S}_{F}=0$. Thus, it appears that no need for extra stabilization (i.e., other than the one already provided by modifying the convective term) of the convective term for the Navier-Stokes equation is necessary for this example, and the previous one.

Next, in order to give a more quantitative comparison of the present approach with previously existing references, we carry out the same verification as in [10]. This is, we consider the horizontal local heat flow given by Nusselt's number, defined as $N u:=\mathbf{u}_{x} \mathrm{t}-\frac{\partial \mathrm{t}}{\partial x}$, where $\mathbf{u}_{x}$ is the velocity's horizontal component. We compute, for $R a=10^{3}, 10^{4}, \ldots, 10^{7}$, the average value of Nusselt's number over the domain $\Omega$, given by

$$
\overline{N u}=\int_{\Omega} N u,
$$

which will be computed considering both the conforming velocity field $\mathbf{u}_{\mathscr{T}}$ and the divergence-free one $\mathscr{L}\left(\mathbf{u}_{\mathscr{T}}, \mathrm{p}_{\mathscr{T}}\right)$. These are denoted as $\overline{N u_{c}}$ and $\overline{N u_{n c}}$, respectively, and are compared with the values obtained in previous references. This comparision is shown in Table 4, where a good agreement is observed between the results given by the present method and the ones reported in the literature.

\begin{tabular}{|c|c|c|c|c|c|c|}
\hline & \multicolumn{2}{|c|}{$\mathcal{S}_{F}(\boldsymbol{\xi}, \zeta)=0$} & \multicolumn{2}{|c|}{$\mathcal{S}_{F}(\xi, \zeta)=\sum_{K \in \mathscr{T}} h_{K} \int_{K} \nabla \cdot \xi \nabla \cdot \zeta$} & \multicolumn{2}{|c|}{$\mathcal{S}_{F}(\boldsymbol{\xi}, \zeta)=\sum_{\gamma \in \mathcal{F}_{I}} h_{\gamma}^{2} \int_{\gamma} \llbracket \partial_{\boldsymbol{n}} \xi_{\mathscr{T}} \rrbracket_{\gamma} \llbracket \partial_{\boldsymbol{n}} \zeta_{\mathscr{T}} \rrbracket_{\gamma}$} \\
\hline Ndof & $\max _{K \in \mathscr{T}}\left|\operatorname{div} \mathbf{u}_{\mathscr{T}}\right|_{K}$ & $\max _{K \in \mathscr{T}}\left|\operatorname{div} \mathscr{L}\left(\mathbf{u}_{\mathscr{T}}, \mathbf{p}_{\mathscr{T}}\right)\right|_{K} \mid$ & $\max _{K \in \mathscr{T}}\left|\operatorname{div} \mathbf{u}_{\mathscr{T}}\right|_{K}$ & $\max _{K \in \mathscr{T}}\left|\operatorname{div} \mathscr{L}\left(\mathbf{u}_{\mathscr{T}}, \mathbf{p}_{\mathscr{T}}\right)\right|_{K} \mid$ & $\max _{K \in \mathscr{T}}\left|\operatorname{div} \mathbf{u}_{\mathscr{T}}\right|_{K}$ & $\max _{K \in \mathscr{T}}\left|\operatorname{div} \mathscr{L}\left(\mathbf{u}_{\mathscr{T}}, \mathrm{p}_{\mathscr{T}}\right)\right|_{K} \mid$ \\
\hline 35 & 26.00 & $1.07 \mathrm{e}-14$ & 23.1 & $2.49 \mathrm{e}-14$ & 14.2 & $1.24 \mathrm{e}-14$ \\
\hline 55 & 14.00 & $7.55 \mathrm{e}-15$ & 12.5 & $9.33 \mathrm{e}-15$ & 9.23 & $5.33 \mathrm{e}-15$ \\
\hline 107 & 4.65 & $6.22 \mathrm{e}-15107$ & 4.39 & $5.33 \mathrm{e}-15$ & 3.79 & $1.07 \mathrm{e}-14$ \\
\hline 187 & 2.00 & $6.22 \mathrm{e}-15187$ & 1.73 & $3.66 \mathrm{e}-15$ & 1.48 & $6.66 \mathrm{e}-15$ \\
\hline 371 & 0.808 & $3.89 \mathrm{e}-15$ & 0.749 & $2.83 \mathrm{e}-15$ & 0.686 & $3.11 \mathrm{e}-15$ \\
\hline 691 & 0.304 & $1.89 \mathrm{e}-15$ & 0.262 & $2 \mathrm{e}-15$ & 0.244 & $2.55 \mathrm{e}-15$ \\
\hline 1379 & 0.128 & $1.72 \mathrm{e}-15$ & 0.119 & $2.03 \mathrm{e}-15$ & 0.114 & $1.6 \mathrm{e}-15$ \\
\hline 2659 & 0.0431 & $1.14 \mathrm{e}-15$ & 0.0383 & $1.17 \mathrm{e}-15$ & 0.0389 & $1.03 \mathrm{e}-15$ \\
\hline 5315 & 0.0164 & $7.22 \mathrm{e}-16$ & 0.0153 & $5.41 \mathrm{e}-16$ & 0.0151 & $6.9 \mathrm{e}-16$ \\
\hline 10435 & 0.0063 & $4.58 \mathrm{e}-16$ & 0.00558 & $3.89 \mathrm{e}-16$ & 0.00562 & $7.49 \mathrm{e}-16$ \\
\hline 20867 & 0.00222 & $4.16 \mathrm{e}-16$ & 0.00204 & $3.33 \mathrm{e}-16$ & 0.00206 & $8.33 \mathrm{e}-16$ \\
\hline 41347 & 0.000804 & $2.5 \mathrm{e}-16$ & 0.000706 & $3.4 \mathrm{e}-16$ & 0.000712 & $6.11 \mathrm{e}-16$ \\
\hline 82692 & 0.000283 & $1.96 \mathrm{e}-16$ & 0.000259 & $2.5 \mathrm{e}-16$ & 0.00026 & $3.97 \mathrm{e}-16$ \\
\hline 164611 & 0.000103 & $1.21 \mathrm{e}-16$ & $9 \mathrm{e}-05$ & $1.57 \mathrm{e}-16$ & $9.05 \mathrm{e}-05$ & $4.69 \mathrm{e}-16$ \\
\hline 329220 & $3.59 \mathrm{e}-05$ & $1.35 \mathrm{e}-16$ & $3.28 \mathrm{e}-05$ & $1.67 \mathrm{e}-16$ & $3.29 \mathrm{e}-05$ & $3.47 \mathrm{e}-16$ \\
\hline 656900 & $1.3 \mathrm{e}-05$ & $9.71 \mathrm{e}-17$ & $1.14 \mathrm{e}-05$ & $1.36 \mathrm{e}-16$ & $1.14 \mathrm{e}-05$ & $3.23 \mathrm{e}-16$ \\
\hline
\end{tabular}

Table 2: Maximum absolute value of the divergence of the solution $\mathbf{u}_{\mathscr{T}}$ and its divergence-free counterpart $\mathscr{L}\left(\mathbf{u}_{\mathscr{T}}, \mathrm{p}_{\mathscr{T}}\right)$ for Example 3 , computed with all the different choices for the stabilizing term $\mathcal{S}_{F}$ and fixing a CIP-type stabilization for $\mathcal{S}_{T}$. 


\begin{tabular}{|c|c|c|c|c|c|c|}
\hline & \multicolumn{2}{|c|}{$\mathcal{S}_{F}(\boldsymbol{\xi}, \boldsymbol{\zeta})=0$} & \multicolumn{2}{|c|}{$\mathcal{S}_{F}(\boldsymbol{\xi}, \boldsymbol{\zeta})=\sum_{K \in \mathscr{T}} h_{K} \int_{K} \nabla \cdot \boldsymbol{\xi} \nabla \cdot \boldsymbol{\zeta}$} & \multicolumn{2}{|c|}{$\mathcal{S}_{F}(\boldsymbol{\xi}, \boldsymbol{\zeta})=\sum_{\gamma \in \mathcal{F}_{I}} h_{\gamma}^{2} \int_{\gamma} \llbracket \partial_{\boldsymbol{n}} \boldsymbol{\xi}_{\mathscr{T}} \rrbracket_{\gamma} \llbracket \partial_{\boldsymbol{n}} \boldsymbol{\zeta}_{\mathscr{T}} \rrbracket_{\gamma}$} \\
\hline Ndof & $\max _{K \in \mathscr{T}}|\operatorname{div} \mathbf{u} \mathscr{T}|_{K}$ & $\max _{K \in \mathscr{T}}\left|\operatorname{div} \mathscr{L}\left(\mathbf{u}_{\mathscr{T}}, \mathrm{p}_{\mathscr{T}}\right)\right|_{K} \mid$ & $\max _{K \in \mathscr{T}}|\operatorname{div} \mathbf{u}|_{K}$ & $\max _{K \in \mathscr{T}}\left|\operatorname{div} \mathscr{L}\left(\mathbf{u}_{\mathscr{T}}, \mathrm{p}_{\mathscr{T}}\right)\right|_{K} \mid$ & $\max _{K \in \mathscr{T}}|\operatorname{div} \mathbf{u} \mathscr{T}|_{K}$ & $\max _{K \in \mathscr{T}}\left|\operatorname{div} \mathscr{L}\left(\mathbf{u}_{\mathscr{T}}, \mathrm{p} \mathscr{T}\right)\right|_{K} \mid$ \\
\hline 35 & 19.9 & $7.11 \mathrm{e}-15$ & 17.6 & $1.42 \mathrm{e}-14$ & 10.5 & $8.88 \mathrm{e}-15$ \\
\hline 55 & 10.5 & $7.55 \mathrm{e}-15$ & 9.1 & $4.66 \mathrm{e}-15$ & 6.56 & $2.66 \mathrm{e}-15$ \\
\hline 107 & 3.45 & $4.55 \mathrm{e}-15$ & 3.27 & $8.88 \mathrm{e}-15$ & 2.83 & $5.11 \mathrm{e}-15$ \\
\hline 187 & 1.57 & $8.88 \mathrm{e}-15$ & 1.31 & $3.89 \mathrm{e}-15$ & 1.12 & $3.94 \mathrm{e}-15$ \\
\hline 371 & 0.632 & $3.33 e-15$ & 0.578 & $3.55 \mathrm{e}-15$ & 0.533 & $2.78 \mathrm{e}-15$ \\
\hline 691 & 0.237 & $2.33 \mathrm{e}-15$ & 0.198 & $1.89 \mathrm{e}-15$ & 0.185 & $1.78 \mathrm{e}-15$ \\
\hline 1379 & 0.102 & $1.22 \mathrm{e}-15$ & 0.0934 & $1.26 \mathrm{e}-15$ & 0.0901 & $1.44 \mathrm{e}-15$ \\
\hline 2659 & 0.0323 & $9.16 \mathrm{e}-16$ & 0.0273 & $8.6 \mathrm{e}-16$ & 0.0282 & $7.49 \mathrm{e}-16$ \\
\hline 5315 & 0.0131 & $5.41 \mathrm{e}-16$ & 0.0121 & $7.36 \mathrm{e}-16$ & 0.0119 & $6.11 \mathrm{e}-16$ \\
\hline 10435 & 0.0047 & $3.89 \mathrm{e}-16$ & 0.00408 & $5 e-16$ & 0.00415 & $7.49 \mathrm{e}-16$ \\
\hline 20867 & 0.0017 & $3.33 e-16$ & 0.00154 & $3.61 \mathrm{e}-16$ & 0.00156 & $5 e-16$ \\
\hline 41347 & 0.000602 & $2.22 \mathrm{e}-16$ & 0.000518 & $2.6 \mathrm{e}-16$ & 0.000525 & $3.75 \mathrm{e}-16$ \\
\hline 82692 & 0.000215 & $1.73 e-16$ & 0.000194 & $2.15 \mathrm{e}-16$ & 0.00020 & $3.61 \mathrm{e}-16$ \\
\hline 164611 & $7.69 \mathrm{e}-05$ & $1.46 \mathrm{e}-16$ & $6.6 \mathrm{e}-05$ & $2.12 \mathrm{e}-16$ & $6.66 \mathrm{e}-05$ & $2.98 \mathrm{e}-16$ \\
\hline 329220 & $2.74 \mathrm{e}-05$ & $1.53 e-16$ & $2.46 \mathrm{e}-05$ & $1.7 \mathrm{e}-16$ & $2.48 \mathrm{e}-05$ & $3.89 \mathrm{e}-16$ \\
\hline 656900 & $9.73 \mathrm{e}-06$ & $1.04 \mathrm{e}-16$ & $8.33 \mathrm{e}-06$ & $1.32 \mathrm{e}-16$ & $8.37 \mathrm{e}-06$ & $2.84 \mathrm{e}-16$ \\
\hline
\end{tabular}

Table 3: Maximum absolute value of the divergence of the solution $\mathbf{u}_{\mathscr{T}}$ and its divergence-free counterpart $\mathscr{L}\left(\mathbf{u}_{\mathscr{T}}, \mathrm{p}_{\mathscr{T}}\right)$ for Example 4 , computed with all the different choices for the stabilizing term $\mathcal{S}_{F}$ and fixing a CIP-type stabilization for $\mathcal{S}_{T}$.

\begin{tabular}{|c|c|c|c|c|c|c|c|c|}
\hline$R a$ & $\overline{N u_{c}}$ & $\overline{N u_{n c}}$ & {$[10]$} & {$[7]$} & {$[8]$} & {$[9]$} & {$[11]$} & {$[12]$} \\
\hline $10^{3}$ & 1.117 & 1.117 & 1.118 & 1.118 & 1.117 & 1.074 & 1.117 & 1.112 \\
\hline $10^{4}$ & 2.240 & 2.241 & 2.245 & 2.243 & 2.243 & 2.084 & 2.254 & 2.198 \\
\hline $10^{5}$ & 4.499 & 4.504 & 4.524 & 4.519 & 4.521 & 4.300 & 4.598 & 4.465 \\
\hline $10^{6}$ & 8.701 & 8.719 & 8.852 & 8.800 & 8.806 & 8.743 & 8.976 & 8.783 \\
\hline $10^{7}$ & 16.489 & 16.491 & 16.789 & - & 16.400 & 13.99 & 16.656 & 16.46 \\
\hline
\end{tabular}

Table 4: The table compares the value of the Nusselt's number for $\mathbf{u}_{\mathscr{T}}$ and $\mathscr{L}\left(\mathbf{u}_{\mathscr{T}}, \mathrm{p} \mathscr{T}\right)$, for Example 4 considering several Rayleigh's number values.

\section{Concluding remarks}

In this work we have presented a low-order stabilized finite element method for a generalized steady-state Boussinesq problem. The main ingredient of the method is the use of the pressure jumps to build a solenoidal discrete velocity that is fed into the momentum and temperature equations. This plays a fundamental role in our stability analysis, since it allows us to prove stability without the need of write the convective terms in a skew-symemtric way. In addition, numerical experiments show that the solenoidal velocity thus obtained recovers features of the exact solution that are not present in the conforming solution.

Since the convective terms do not need to be rewritten, it is our impression that the application of this idea to the time dependent case, treated in the very recent papers $[17,10]$ where the convective terms are written in the skewsymmetric way, could simplify the analysis greatly in that case as well. In addition, since in this paper the interest was the steady-state case, the need of the Hypothesis (34) appeared. This restriction is likely not to be needed in the time dependent case (as the results in $[17,10]$ indicate), which, again, would generalize the results and simplify the analysis. The extension to the time dependent case, as well as also more theoretical aspects such as conditions under which the solution is unique, will be the topic of future research.

\section{Appendix: Technical Proofs}

This section is devoted to provide the proof of some technical results from Section 4.1. 


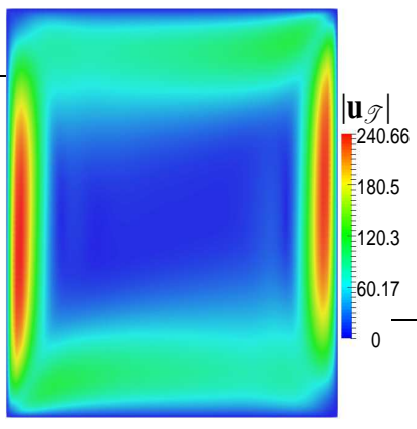

(a.1)

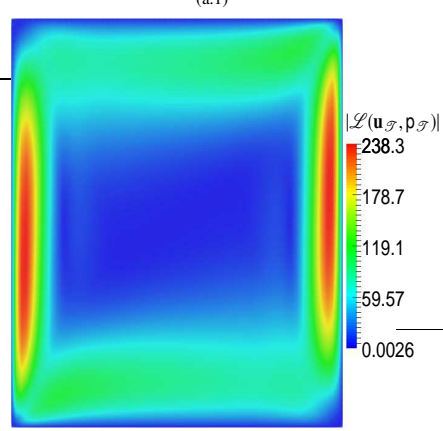

(b.1)

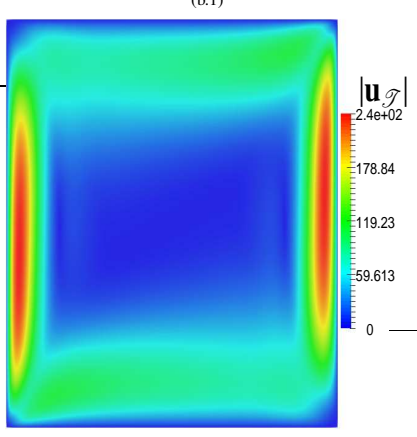

(a.2)

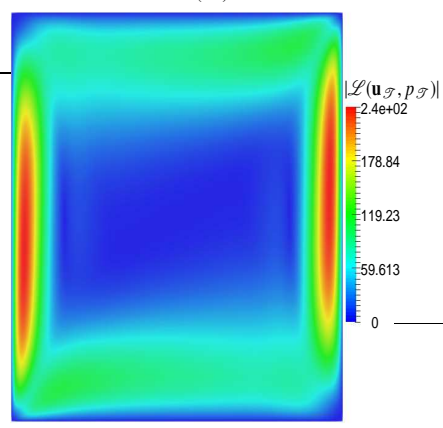

(b.2)

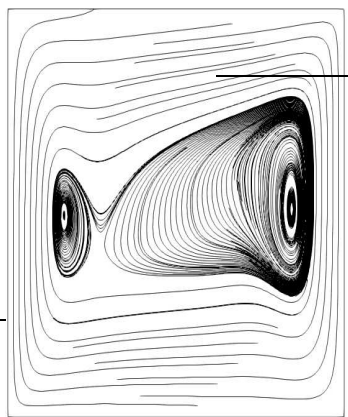

(c.1)

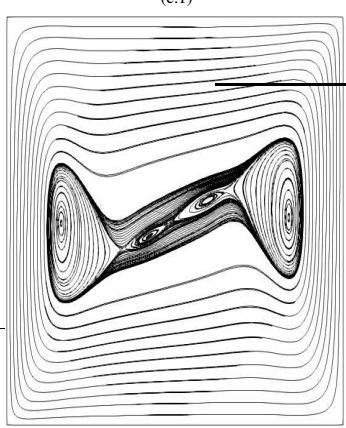

(d.1)

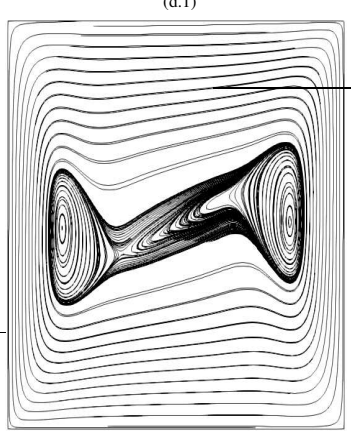

(c.2)

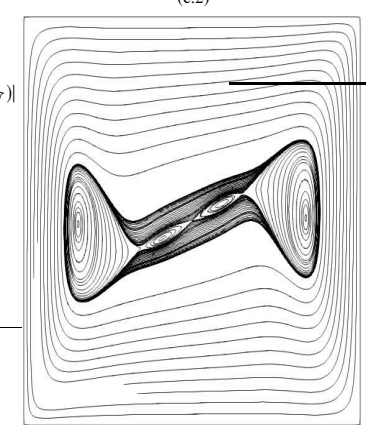

(d.2)

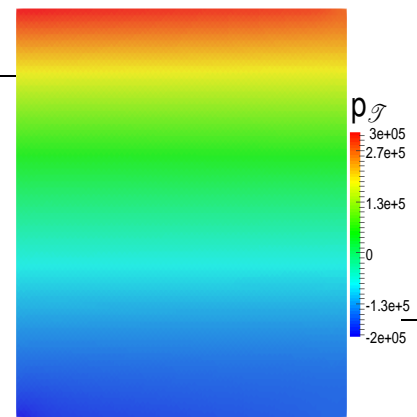

(e.1)

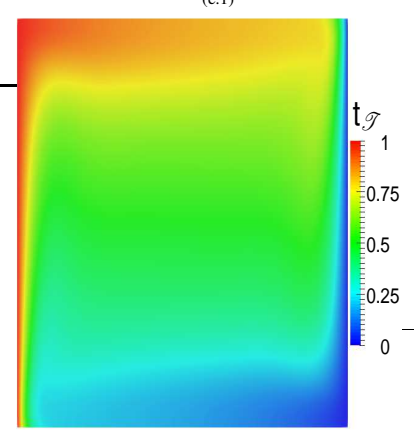

(f.1)

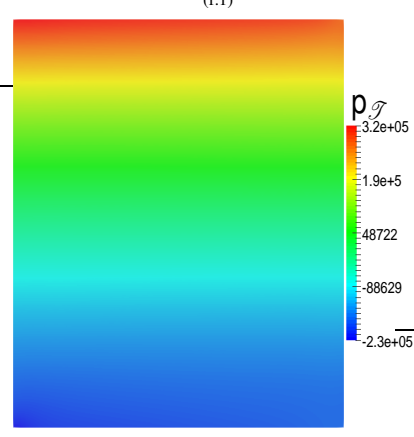

(e.2)

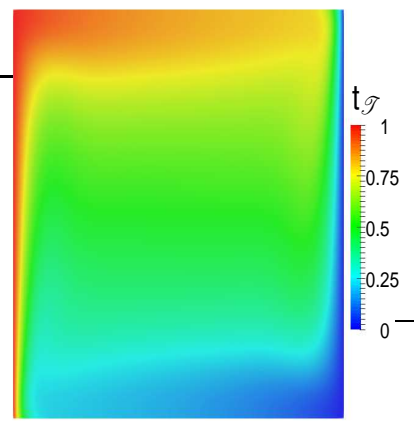

(f.2)

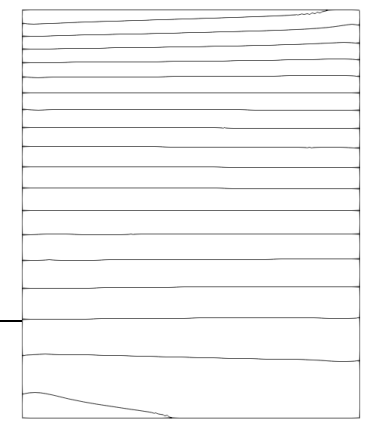

(g.1)

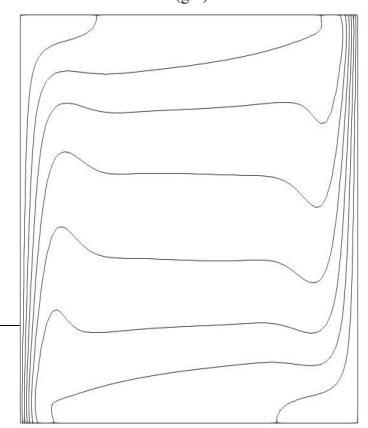

(h.1)

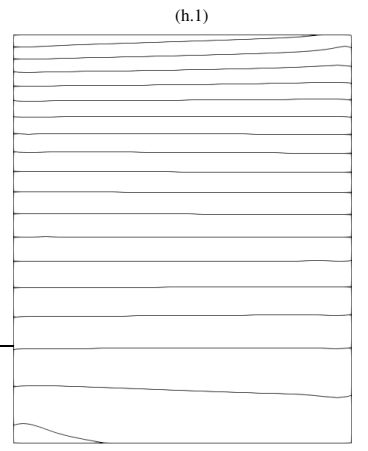

(g.2)

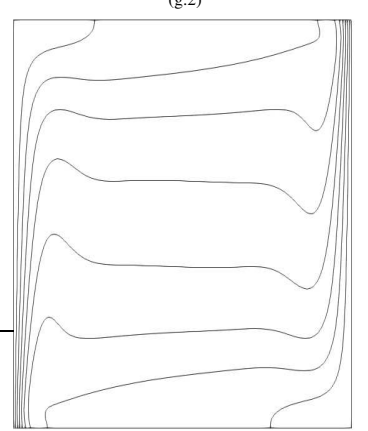

(h.2)

Figure 6: Finite element solution for Example 3, where $R a=10^{6}$ : From (a.1) to (h.1) the mesh contains 8321 nodes and 16384 elements; from (a.2) to (h.2) analogous images are shown for a mesh with 263169 nodes and 524288 elements. (a.) and (b.) are the graphic representations of $|\mathbf{u} \mathscr{T}|$ and $\left|\mathscr{L}\left(\mathbf{u} \mathscr{T}, p_{\mathscr{T}}\right)\right|$, respectively; (c.) and (d.) show streamlines for the fields $\mathbf{u} \mathscr{T}$ and $\mathscr{L}\left(\mathbf{u} \mathscr{T}, p_{\mathscr{T}}\right)$, respectively; (e.) and (f.) show the graphic representations of $p_{\mathscr{T}}$ and $\mathrm{t} \mathscr{T}$, respectively; (g.) and (h.) show the contour lines of $p_{\mathscr{T}}$ and $\mathrm{t} \mathscr{T}$ respectively. 


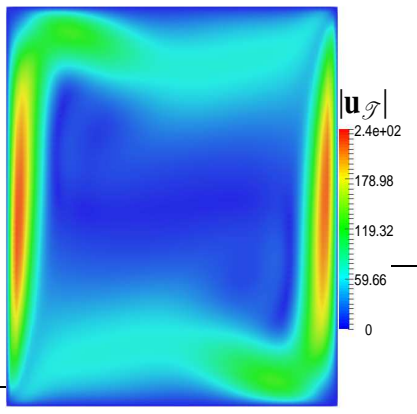

(a.1)

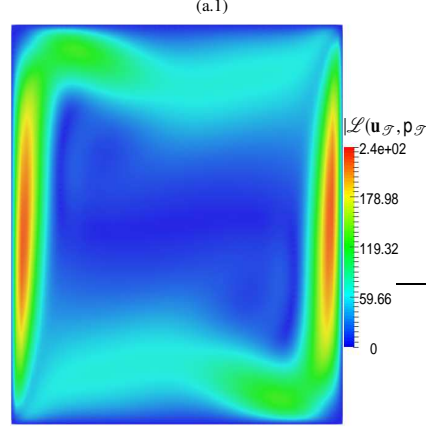

(b.1)

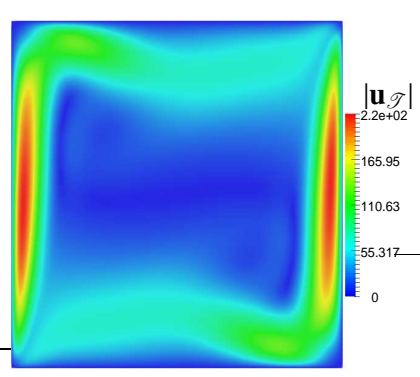

(a.2)

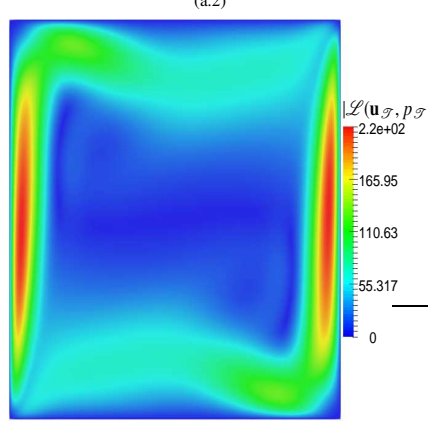

(b.2)

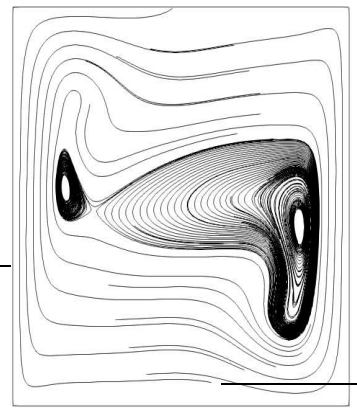

(c.1)

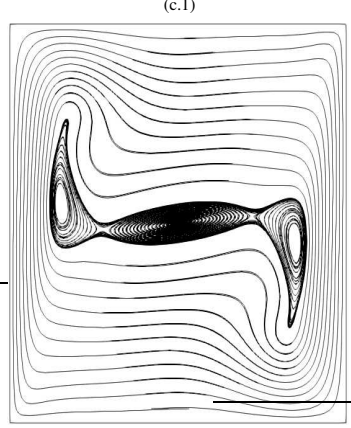

(d.1)

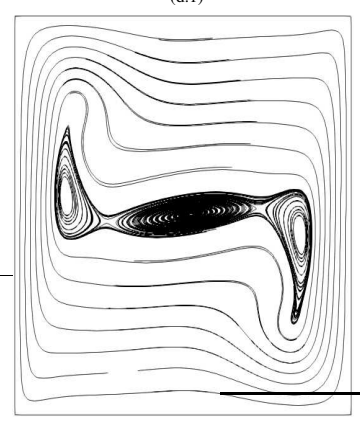

(c.2)

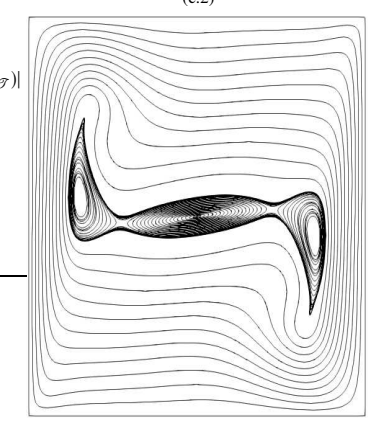

(d.2)

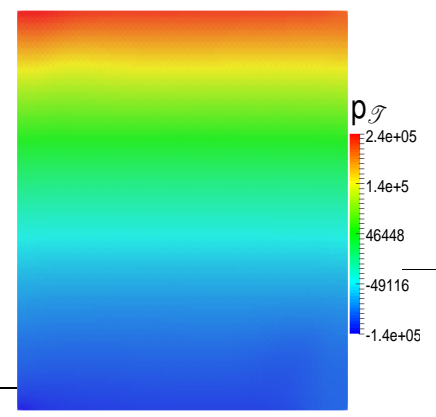

(e.1)

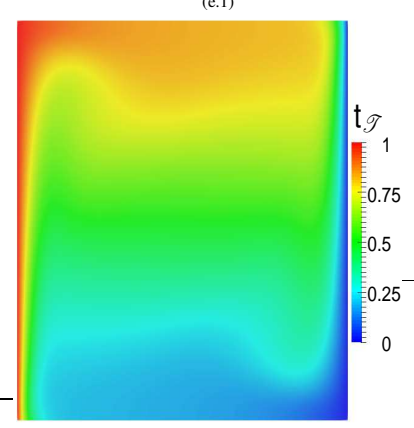

(f.1)

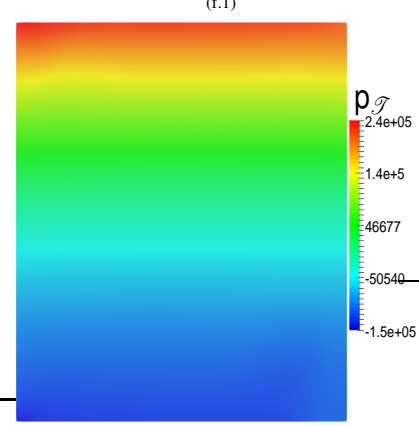

(e.2)

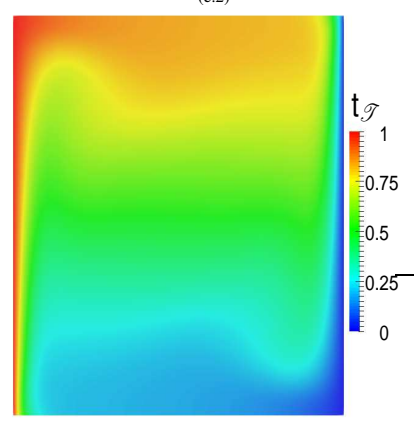

(f.2)

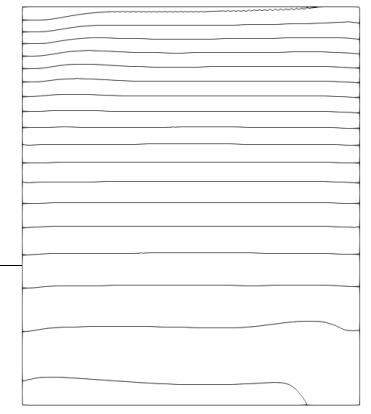

(g.1)

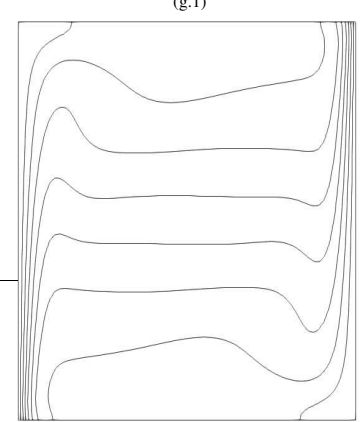

(h.1)

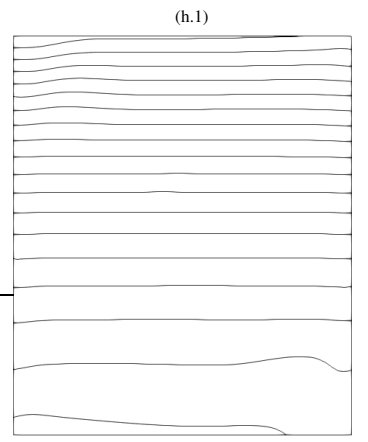

(g.2)

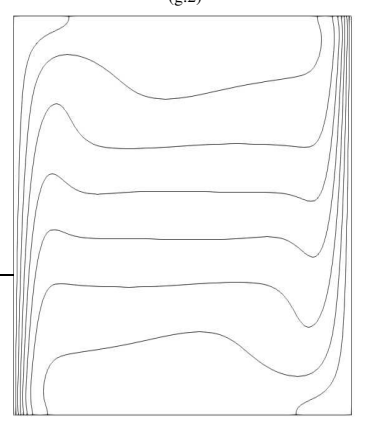

(h.2)

Figure 7: Finite element solution for Example 3, where $R a=10^{6}$ : From (a.1) to (h.1) the mesh consists of 8321 nodes and 16384 elements; from (a.2) to (h.2) analogous images are shown for a mesh with 263169 nodes and 524288 elements. (a.) and (b.) are the graphic representations of $\left|\mathbf{u}_{\mathscr{T}}\right|$ and $\left|\mathscr{L}\left(\mathbf{u}_{\mathscr{T}}, p_{\mathscr{T}}\right)\right|$, respectively; (c.) and (d.) show streamlines for the fields $\mathbf{u}_{\mathscr{T}}$ and $\mathscr{L}\left(\mathbf{u}_{\mathscr{T}}, p_{\mathscr{T}}\right)$, respectively; (e.) and (f.) show the graphic representations of $p_{\mathscr{T}}$ and $\mathrm{t}_{\mathscr{T}}$, respectively; (g.) and (h.) show the contour lines of $p_{\mathscr{T}}$ and $\mathrm{t}_{\mathscr{T}}$ respectively. 


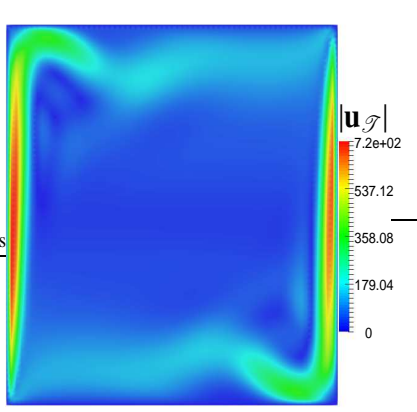

(a.1)

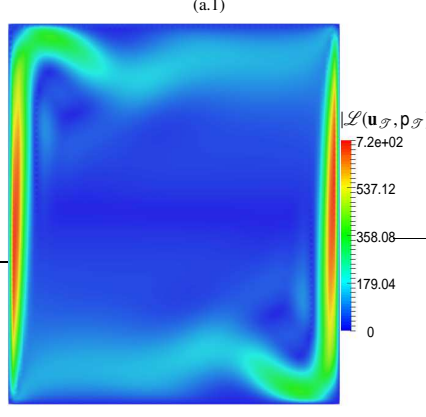

(b.1)

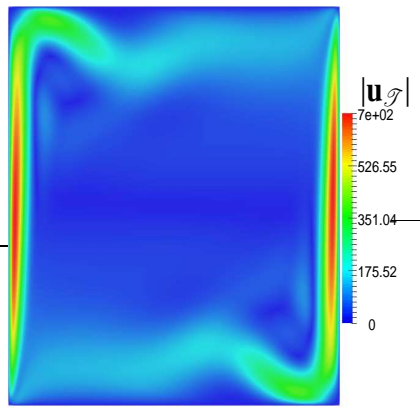

(a.2)

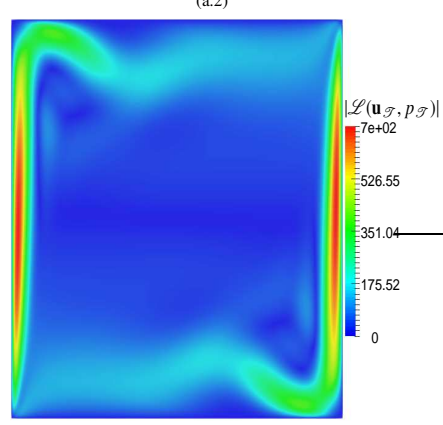

(b.2)

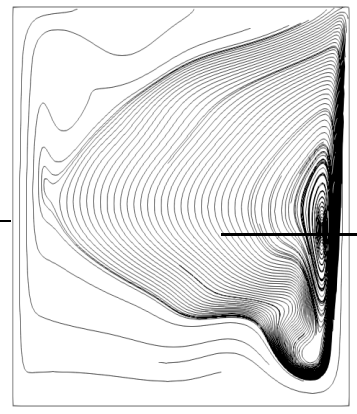

(c.1)

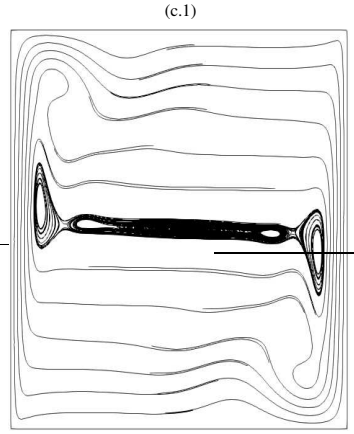

(d.1)

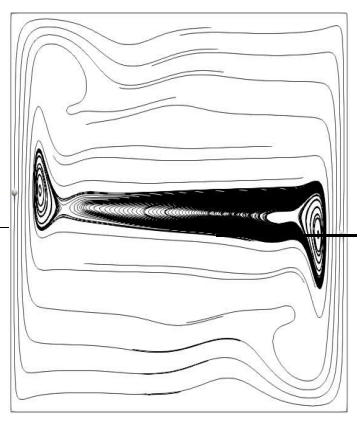

(c.2)

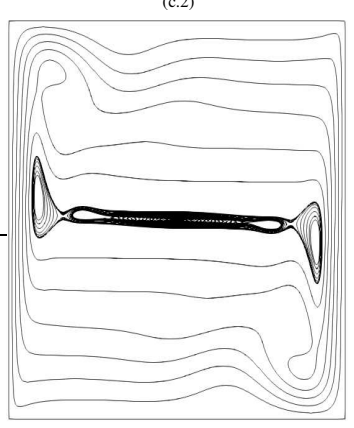

(d.2)

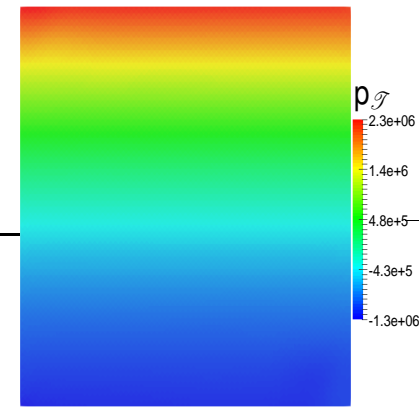

(e.1)

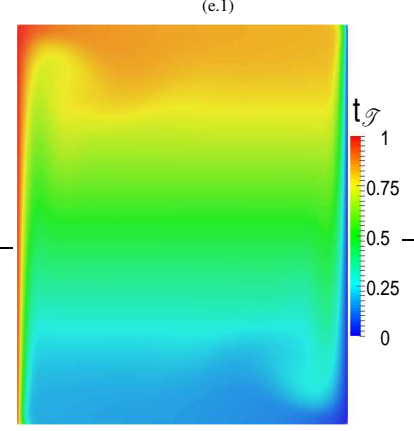

(f.1)

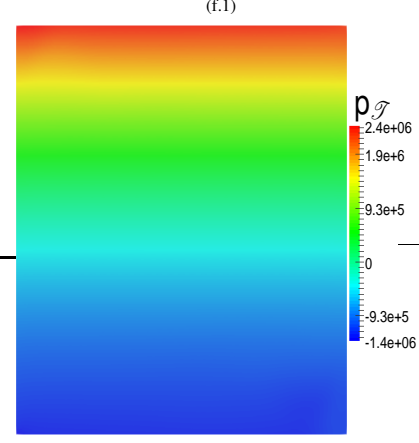

(e.2)

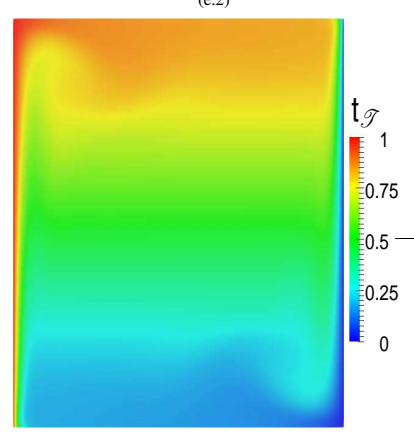

(f.2)

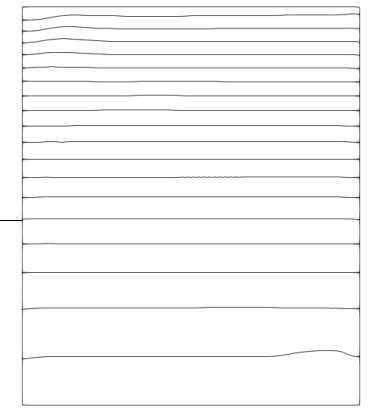

(g.1)

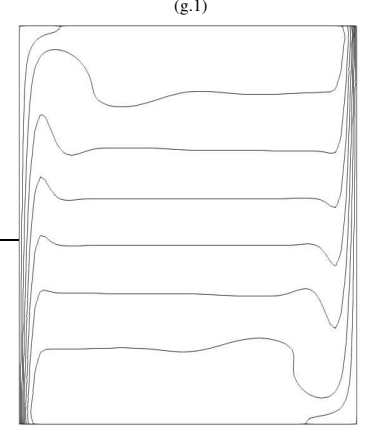

(h.1)

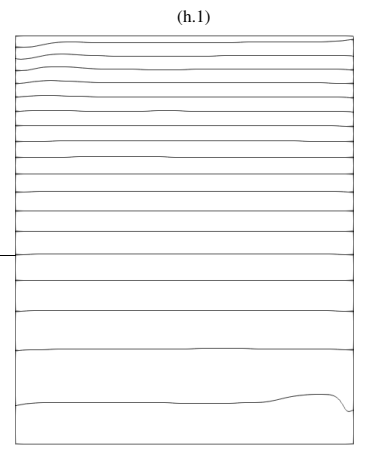

(g.2)

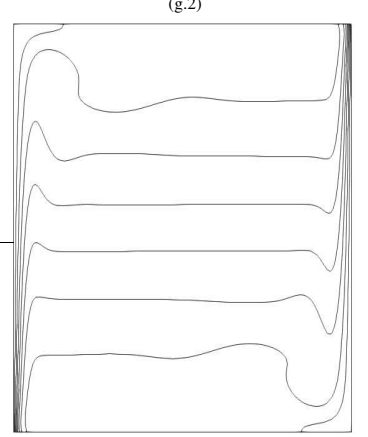

(h.2)

Figure 8: Finite element solution for Example 4, where $R a=10^{7}$ : From (a.1) to (h.1) the mesh contains 8321 nodes and 16384 elements; from (a.2) to (h.2) analogous images are shown for a mesh with 263169 nodes and 524288 elements. (a.) and (b.) are the graphic representations of $|\mathbf{u} \mathscr{T}|$ and $|\mathscr{L}(\mathbf{u} \mathscr{T}, p \mathscr{T})|$, respectively; (c.) and (d.) show streamlines for the fields $\mathbf{u}_{\mathscr{T}}$ and $\mathscr{L}(\mathbf{u} \mathscr{T}, p \mathscr{T})$, respectively; (e.) and (f.) show the graphic representations of $p_{\mathscr{T}}$ and $\mathrm{t}_{\mathscr{T}}$, respectively; (g.) and (h.) show the contour lines of $p_{\mathscr{T}}$ and $\mathrm{t} \mathscr{T}$ respectively. 
Proof of Lemma 3. To prove (22), we use (17). In fact, for any $\boldsymbol{\xi} \in \boldsymbol{H}_{0}^{1}(\Omega), \phi_{\mathscr{T}} \in Q(\mathscr{T})$ and $K \in \mathscr{T}$, it follows that

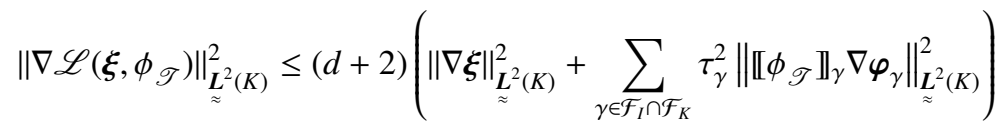

$$
\begin{aligned}
& \leq C\left(\|\nabla \boldsymbol{\xi}\|_{\boldsymbol{\sim}^{2}(K)}^{2}+\sum_{\gamma \in \mathcal{F}_{I} \cap \mathcal{F}_{K}} \tau_{\gamma}^{2} \llbracket \phi_{\mathscr{T}} \mathbb{1}_{\gamma}^{2} h_{K}^{d-2}\right)
\end{aligned}
$$

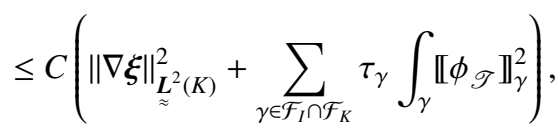

upon using the mesh regularity, (1) and (16). Then, (22) follows by adding over all the elements in the mesh.

To prove (23), we consider the broken Sobolev embedding given in [15, Equation 3.12] together with (22), and obtain

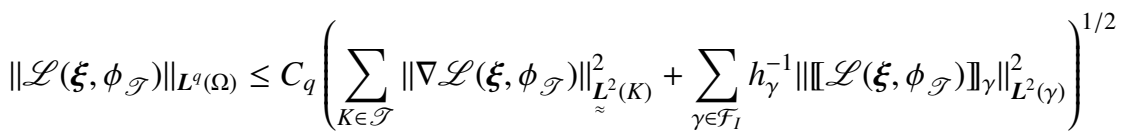

$$
\begin{aligned}
& \leq C_{q}\left(C_{1}\left(\|\nabla \boldsymbol{\xi}\|_{\boldsymbol{L}^{2}(\Omega)}^{2}+\sum_{\gamma \in \mathcal{F}_{I}} \tau_{\gamma}\left\|\llbracket \phi_{\mathscr{T}}\right\| \|_{L^{2}(\gamma)}^{2}\right)+\sum_{\gamma \in \mathcal{F}_{I}} h_{\gamma}^{-1}\left\|\llbracket \mathscr{L}\left(\xi, \phi_{\mathscr{T}}\right)\right\|_{\gamma} \|_{\boldsymbol{L}^{2}(\gamma)}^{2}\right)^{1 / 2}
\end{aligned}
$$

Now, using (17), and the fact that $\llbracket \xi \rrbracket_{\gamma}=0$ (since $\boldsymbol{\xi} \in \boldsymbol{V}(\mathscr{T})$ ), we arrive at

$$
\left\|\mathscr{L}\left(\boldsymbol{\xi}, \phi_{\mathscr{T}}\right)\right\|_{L^{q}(\Omega)} \leq C_{q}\left(C_{1}\left(\|\nabla \boldsymbol{\xi}\|_{\boldsymbol{L}^{2}(\Omega)}^{2}+\sum_{\gamma \in \mathcal{F}_{I}} \tau_{\gamma}\left\|\llbracket \phi_{\mathscr{T}} \rrbracket\right\|_{L^{2}(\gamma)}^{2}\right)+\sum_{\gamma \in \mathcal{F}_{I}} h_{\gamma}^{-1}\|\| \sum_{\gamma^{\prime} \in \mathcal{F}_{I} \cap \Omega_{\gamma}} \tau_{\gamma^{\prime}} \llbracket \phi_{\mathscr{T}} \rrbracket_{\gamma^{\prime}} \boldsymbol{\varphi}_{\gamma^{\prime}}\left\|_{\gamma}\right\|_{L^{2}(\gamma)}^{2}\right)^{1 / 2}
$$

In addition, it is not difficult to see that

$$
\sum_{\gamma \in \mathcal{F}_{I}} h_{\gamma}^{-1}\|\| \sum_{\gamma^{\prime} \in \mathcal{F}_{I} \cap \Omega_{\gamma}} \tau_{\gamma^{\prime}} \llbracket \phi_{\mathscr{T}} \rrbracket_{\gamma^{\prime}} \boldsymbol{\varphi}_{\gamma^{\prime}}\left\|_{\gamma}\right\|_{L^{2}(\gamma)}^{2} \leq C_{2} \sum_{\gamma \in \mathcal{F}_{I}} h_{\gamma}^{-1} \sum_{\gamma^{\prime} \in \mathcal{F}_{I} \cap \Omega_{\gamma}} \tau_{\gamma^{\prime}}^{2} \llbracket \phi_{\mathscr{T}} \mathbb{1}_{\gamma^{\prime}}^{2}\left\|\llbracket \boldsymbol{\varphi}_{\gamma^{\prime}} \rrbracket_{\gamma}\right\|_{L^{2}(\gamma)}^{2},
$$

Then, replacing the last result in (80), gives

$$
\begin{aligned}
\left\|\mathscr{L}\left(\boldsymbol{\xi}, \phi_{\mathscr{T}}\right)\right\|_{L^{q}(\Omega)} & \leq C_{q}\left(C_{1}\left(\|\nabla \boldsymbol{\xi}\|_{\underset{L^{2}(\Omega)}{2}}^{2}+\sum_{\gamma \in \mathcal{F}_{I}} \tau_{\gamma}\left\|\llbracket \phi_{\mathscr{T}} \rrbracket\right\|_{L^{2}(\gamma)}^{2}\right)\right. \\
& \left.+C_{2} \sum_{\gamma \in \mathcal{F}_{I}} h_{\gamma}^{-1} \sum_{\gamma^{\prime} \in \mathcal{F}_{I} \cap \Omega_{\gamma}} \tau_{\gamma^{\prime}}^{2} \llbracket \phi_{\mathscr{T}} \mathbb{1}_{\gamma^{\prime}}^{2}\left\|\llbracket \varphi_{\gamma^{\prime}} \rrbracket_{\gamma}\right\|_{L^{2}(\gamma)}^{2}\right)^{1 / 2},
\end{aligned}
$$

Next, to bound the jump of $\boldsymbol{\varphi}_{\gamma^{\prime}}$, we use the mesh regularity to obtain

$$
\left\|\llbracket \boldsymbol{\varphi}_{\gamma^{\prime}} \rrbracket_{\gamma}\right\|_{\boldsymbol{L}^{2}(\gamma)}^{2}=\left\|\sum_{i=1}^{d}\right\| \frac{\left|\gamma^{\prime}\right|}{d|K|} \lambda_{i}\left(\boldsymbol{x}_{i}-\boldsymbol{x}_{\gamma^{\prime}}\right)\left\|_{\gamma}\right\|_{\boldsymbol{L}^{2}(\gamma)}^{2} \leq C_{3}\left\|\frac{h_{\gamma}^{d-1} h_{\gamma}}{h_{\gamma}^{d}} \sum_{i=1, \ldots, d} \lambda_{i}\right\|_{L^{2}(\gamma)}^{2} \leq C_{3} h_{\gamma}^{d-1} .
$$

Thus, replacing this last bound in (81), and using the mesh regularity, the fact that $\tau_{\gamma}=h_{\gamma} / 12$, and using that 
$\int_{\gamma} \llbracket \phi_{\mathscr{T}} \mathbb{1}_{\gamma}^{2}=|\gamma| \llbracket \phi_{\mathscr{T}} \mathbb{1}_{\gamma}^{2}$ and $|\gamma| \sim h_{\gamma}^{d-1}$, we obtain

$$
\begin{aligned}
\left\|\mathscr{L}\left(\xi, \phi_{\mathscr{T}}\right)\right\|_{L^{q}(\Omega)} & \leq C_{q}\left(C_{1}\left(\|\nabla \boldsymbol{\xi}\|_{\boldsymbol{L}^{2}(\Omega)}^{2}+\sum_{\gamma \in \mathcal{F}_{I}} \tau_{\gamma}\left\|\llbracket \phi_{\mathscr{T}} \mathbb{\|}\right\|_{L^{2}(\gamma)}^{2}\right)+C \sum_{\gamma \in \mathcal{F}_{I}} \tau_{\gamma}^{2} \llbracket \phi_{\mathscr{T}} \mathbb{1}_{\gamma}^{2} h_{\gamma}^{d-2}\right)^{1 / 2} \\
& =C_{q}\left(C_{1}\|\nabla \boldsymbol{\xi}\|_{\underline{\Sigma}^{2}(\Omega)}^{2}+\tilde{C} \sum_{\gamma \in \mathcal{F}_{I}} \tau_{\gamma} \int_{\gamma} \llbracket \phi_{\mathscr{T}} \mathbb{1}_{\gamma}^{2}\right)^{1 / 2} .
\end{aligned}
$$

Therefore, (23) follows with $\bar{C}_{q}=C_{q}\left(\max \left\{C_{1} / \varepsilon_{1}, \tilde{C}\right\}\right)^{1 / 2}$.

Proof of Lemma 4. Inequality (24) is shown applying the Hölder inequality, the Sobolev embedding (4), the Poincaré inequalities given in (4), and the definitions of $\|(\cdot, \cdot)\|_{\mathcal{N}(\mathscr{T})}$ and $\|\cdot\|_{\mathcal{T}(\mathscr{T})}$, with $\tilde{C}_{g}=C_{e m b}^{2} \tilde{C}_{p} \varepsilon_{1}^{-1 / 2} \kappa_{1}^{-1 / 2}$. Inequality (25) is proven applying the triangle inequality, (8), (20), and the definition of $\|\cdot\|_{\mathcal{T}(\mathscr{T})}$, with $\tilde{C}_{\mathcal{A}_{T}}=\frac{\kappa_{2}}{\kappa_{1}}+1$. To prove (26), we use (11) considering that $\mathscr{L}\left(\boldsymbol{\vartheta}_{\mathscr{T}}, \chi_{\mathscr{T}}\right)$ is divergence-free and its normal component vanishes at the boundary $\Gamma$, and then we apply Hölder's inequality to obtain

$$
\left|\mathcal{C}_{F}\left(\mathscr{L}\left(\boldsymbol{\vartheta}_{\mathscr{T}}, \chi_{\mathscr{T}}\right) ; \boldsymbol{\xi}_{\mathscr{T}}, \zeta_{\mathscr{T}}\right)\right|=\left|-C_{F}\left(\mathscr{L}\left(\boldsymbol{\vartheta}_{\mathscr{T}}, \chi_{\mathscr{T}}\right) ; \zeta_{\mathscr{T}}, \boldsymbol{\xi}_{\mathscr{T}}\right)\right| \leq\left\|\mathscr{L}\left(\boldsymbol{\vartheta}_{\mathscr{T}}, \chi_{\mathscr{T}}\right)\right\|_{L^{6}(\Omega)}\left\|\nabla \zeta_{\mathscr{T}}\right\|_{\tilde{L}^{2}(\Omega)}\left\|\boldsymbol{\xi}_{\mathscr{T}}\right\|_{L^{3}(\Omega)}
$$

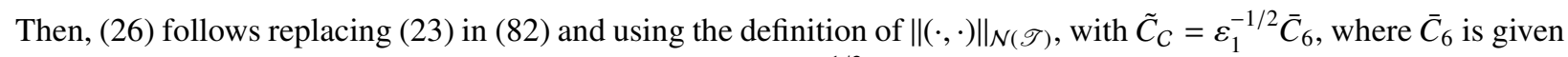
in (23). Almost identical calculations prove (27) with $\tilde{C}_{\mathfrak{c}}=\kappa_{1}^{-1 / 2} \bar{C}_{6}$, where $\bar{C}_{6}$ is the constant from (23). The next two results are proven in a similar way as the one for (26) and (27). From using (11) and (13) and the Hölder inequality, we get

$$
\begin{aligned}
& \left|\mathcal{C}_{F}\left(\mathscr{L}(\boldsymbol{\vartheta}, \chi) ; \xi, \zeta_{\mathscr{T}}\right)\right| \leq\|\mathscr{L}(\boldsymbol{\vartheta}, \chi)\|_{L^{2}(\Omega)}\left\|\nabla \zeta_{\mathscr{T}}\right\|_{\tilde{\sim}^{2}(\Omega)}\|\boldsymbol{\xi}\|_{L^{\infty}(\Omega)}, \\
& \left|C_{T}\left(\mathscr{L}(\boldsymbol{\vartheta}, \chi) ; \theta, \psi_{\mathscr{T}}\right)\right| \leq\|\mathscr{L}(\boldsymbol{\vartheta}, \chi)\|_{L^{2}(\Omega)}\left\|\nabla \psi_{\mathscr{T}}\right\|_{L^{2}(\Omega)}\|\theta\|_{L^{\infty}(\Omega)} .
\end{aligned}
$$

Then, (28) and (29) follow from applying (23) and considering the definitions of the mesh-dependent norms, with $\tilde{C}_{C 2}=\varepsilon_{1}^{-1 / 2} \bar{C}_{2}$ and $\tilde{C}_{\mathrm{c} 2}=\kappa_{1}^{-1 / 2} \bar{C}_{2}$, where $\bar{C}_{2}$ is the constant from (23). To prove (30), we use (8) and the Hölder inequality to obtain

$$
\left|\mathcal{A}_{F}\left(\psi_{1} ; \boldsymbol{\xi}, \zeta\right)-\mathcal{A}_{F}\left(\psi_{2} ; \boldsymbol{\xi}, \zeta\right)\right| \leq C_{\varepsilon, l i p}\left\|\psi_{1}-\psi_{2}\right\|_{L^{2}(\Omega)}\|\nabla \boldsymbol{\xi}\|_{\boldsymbol{L}^{\infty}(\Omega)}\|\nabla \zeta\|_{\mathbf{L}^{2}(\Omega)} .
$$

Then, we can get (30) from considering the definitions of the mesh-dependent norms, with $C_{\varepsilon}=\left(\varepsilon_{1} \kappa_{1}\right)^{-1 / 2}$. We can obtain (31) following analogous steps; thus, we have that result with $C_{\kappa}=\kappa_{1}^{-1}$. Finally, (32) follows using (8) and the definition of the $\|(\cdot, \cdot)\|_{\mathcal{N}(\mathscr{T})}$ norm, and (33) follows from using (8), the definition of the $\|\cdot\|_{\mathcal{T}(\mathscr{T})}$ norm and the Poincaré inequality (4), with $\tilde{C}_{c o, T}=\min \left\{1, \tilde{C}_{p}^{-2}\right\}$.

\section{Acknowledgements}

The first author was supported by the Fondecyt Grant $\mathrm{N}^{o} 1170579$. The second author was partially supported by CONICYT-Chile throught Project MEC 80140118. The third author was supported by the CONICYT PFCHA/MagiísterNacional/201 22162137

[1] D. Tritton, Physical Fluid Dynamics, 2nd edition, JOxford University Press, New York, 1988.

[2] R. K. Zeytounian, Joseph boussinesq and his approximation: a contemporary view, C. R. Mecanique 331 (2003) $575-586$.

[3] J. Boland, W. Layton, An analysis of the finite element method for natural convection problems, Numer. Methods Partial Differential Equations 6 (2) (1990) 115-126.

[4] C. Bernardi, B. Métivet, B. Pernaud-Thomas, Couplage des équations de Navier-Stokes et de la chaleur: le modèle et son approximation par éléments finis, RAIRO Modél. Math. Anal. Numér. 29 (7) (1995) 871-921.

[5] C. E. Pérez, J.-M. Thomas, S. Blancher, R. Creff, The steady Navier-Stokes/energy system with temperature-dependent viscosity. I. Analysis of the continuous problem, Internat. J. Numer. Methods Fluids 56 (1) (2008) 63-89.

[6] C. E. Pérez, J.-M. Thomas, S. Blancher, R. Creff, The steady Navier-Stokes/energy system with temperature-dependent viscosity. II. The discrete problem and numerical experiments, Internat. J. Numer. Methods Fluids 56 (1) (2008) 91-114. 
[7] G. de Vahl Davis, Natural convection of air in a square cavity: a bench mark numerical solution, International Journal for numerical methods in fluids 3 (3) (1983) 249-264.

[8] N. Massarotti, P. Nithiarasu, O. Zienkiewicz, Characteristic-based-split (cbs) algorithm for incompressible flow problems with heat transfer, International Journal of Numerical Methods for Heat \& Fluid Flow 8 (8) (1998) 969-990.

[9] M. T. Manzari, An explicit finite element algorithm for convection heat transfer problems, International Journal of Numerical Methods for Heat \& Fluid Flow 9 (8) (1999) 860-877.

[10] T. C. Rebollo, M. G. Mármol, F. Hecht, S. Rubino, I. S. Muñoz, A high-order local projection stabilization method for natural convection problems, Journal of Scientific ComputingTo appear.

[11] D. Wan, B. Patnaik, W. Wei, A new benchmark quality solution for the buoyancy-driven cavity by discrete singular convolution, Numerical Heat Transfer: Part B: Fundamentals 40 (3) (2001) 199-228.

[12] M. Benítez, A. Bermúdez, A second order characteristics finite element scheme for natural convection problems, J. Comput. Appl. Math. 235 (11) (2011) 3270-3284.

[13] P. W. Schroeder, G. Lube, Stabilised dG-FEM for incompressible natural convection flows with boundary and moving interior layers on non-adapted meshes, J. Comput. Phys. 335 (2017) 760-779.

[14] S. A. Lorca, J. L. Boldrini, Stationary solutions for generalized Boussinesq models, J. Differential Equations 124 (2) (1996) 389-406

[15] R. Oyarzúa, T. Qin, D. Schötzau, An exactly divergence-free finite element method for a generalized Boussinesq problem, IMA J. Numer. Anal. 34 (3) (2014) 1104-1135.

[16] K. J. Galvin, A. Linke, L. G. Rebholz, N. E. Wilson, Stabilizing poor mass conservation in incompressible flow problems with large irrotational forcing and application to thermal convection, Comput. Methods Appl. Mech. Engrg. 237/240 (2012) 166-176.

[17] H. Dallmann, D. Arndt, Stabilized finite element methods for the Oberbeck-Boussinesq model, J. Sci. Comput. 69 (1) (2016) $244-273$.

[18] V. John, A. Linke, C. Merdon, M. Neilan, L. G. Rebholz, On the Divergence Constraint in Mixed Finite Element Methods for Incompressible Flows, SIAM Rev. 59 (3) (2017) 492-544.

[19] G. R. Barrenechea, F. Valentin, Consistent local projection stabilized finite element methods, SIAM J. Numer. Anal. 48 (5) (2010) $1801-1825$.

[20] P. G. Ciarlet, The finite element method for elliptic problems, Vol. 40 of Classics in Applied Mathematics, Society for Industrial and Applied Mathematics (SIAM), Philadelphia, PA, 2002, reprint of the 1978 original [North-Holland, Amsterdam; MR0520174 (58 \#25001)]

[21] S. C. Brenner, L. R. Scott, The mathematical theory of finite element methods, 3rd Edition, Vol. 15 of Texts in Applied Mathematics, Springer, New York, 2008

[22] M. Bebendorf, A note on the Poincaré inequality for convex domains, Z. Anal. Anwendungen 22 (4) (2003) 751-756.

[23] S. G. Mikhlin, Constants in some inequalities of analysis, A Wiley-Interscience Publication, John Wiley \& Sons, Ltd., Chichester, 1986, translated from the Russian by Reinhard Lehmann.

[24] L. E. Payne, H. F. Weinberger, An optimal Poincaré inequality for convex domains, Arch. Rational Mech. Anal. 5 (1960) 286-292 (1960).

[25] V. Girault, P.-A. Raviart, Finite element methods for Navier-Stokes equations, Vol. 5 of Springer Series in Computational Mathematics, Springer-Verlag, Berlin, 1986, theory and algorithms.

[26] S. Horn, O. Shishkina, Rotating non-Oberbeck-Boussinesq Rayleigh-Bénard convection in water, Phys. Fluids 26 (2014) 055111.

[27] D. Kizildag, I. Rodríguez, A. Oliva, O. Lehmkuhl, Limits of the Oberbeck-Boussinesq approximation in a tall differentially heated cavity filled with water, Int. J. Heat Mass Transfer 68 (2014) 489-499.

[28] G. R. Barrenechea, F. Valentin, Beyond pressure stabilization: a low-order local projection method for the Oseen equation, Internat. J. Numer. Methods Engrg. 86 (7) (2011) 801-815.

[29] E. Burman, P. Hansbo, Edge stabilization for Galerkin approximations of convection-diffusion-reaction problems, Comput. Methods Appl. Mech. Engrg. 193 (15-16) (2004) 1437-1453.

[30] F. Brezzi, J. Rappaz, P.-A. Raviart, Finite-dimensional approximation of nonlinear problems. II. Limit points, Numer. Math. 37 (1) (1981) $1-28$.

[31] P. Amestoy, I. Duff, J.-Y. L'Excellent, Multifrontal parallel distributed symmetric and unsymmetric solvers, Comput. Methods in Appl. Mech. Eng. 184 (24) (2000) $501-520$.

[32] P. Amestoy, I. Duff, J.-Y. L'Excellent, J. Koster, A fully asynchronous multifrontal solver using distributed dynamic scheduling, SIAM J. Matrix Anal. Appl. 23 (1) (2001) 15-41 (electronic).

[33] M. Braack, E. Burman, V. John, G. Lube, Stabilized finite element methods for the generalized Oseen problem, Comput. Methods Appl. Mech. Engrg. 196 (4-6) (2007) 853-866.

[34] E. W. Jenkins, V. John, A. Linke, L. G. Rebholz, On the parameter choice in grad-div stabilization for the Stokes equations, Adv. Comput. Math. 40 (2) (2014) 491-516.

[35] P. Le Quéré, M. Behnia, From onset of unsteadiness to chaos in a differentially heated square cavity, J. Fluid Mech. 359 (1998) $81-107$. 